\title{
MST
}

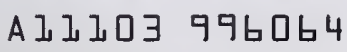

PUBLICATIONS

NIST Monograph 178

Speed of Sound Data and Related Models for Mixtures of Natural Gas Constituents

B.A. Younglove, N.V. Frederick, and R.D. McCarty

QC

100

.0556

$\# 173$

1993

United States Department of Commerce

Technology Administration

National Institute of Standards and Technology 
he National Institute of Standards and Technology was established in 1988 by Congress to "assist industry in the development of technology ... . needed to improve product quality, to modernize manufacturing processes, to ensure product reliability ... and to facilitate rapid commercialization ... of products based on new scientific discoveries."

NIST, originally founded as the National Bureau of Standards in 1901, works to strengthen U.S. industry's competitiveness; advance science and engineering; and improve public health, safety, and the environment. One of the agency's basic functions is to develop, maintain, and retain custody of the national standards of measurement, and provide the means and methods for comparing standards used in science, engineering, manufacturing, commerce, industry, and education with the standards adopted or recognized by the Federal Government.

As an agency of the U.S. Commerce Department's Technology Administration, NIST conducts basic and applied research in the physical sciences and engineering and performs related services. The Institute does generic and precompetitive work on new and advanced technologies. NIST's research facilities are located at Gaithersburg, MD 20899, and at Boulder, CO 80303. Major technical operating units and their principal activities are listed below. For more information contact the Public Inquiries Desk, 301-975-3058.

\section{Technology Services}

- Manufacturing Technology Centers Program

- Standards Services

- Technology Commercialization

- Measurement Services

- Technology Evaluation and Assessment

- Information Services

Electronics and Electrical Engineering Laboratory

- Microelectronics

- Law Enforcement Standards

- Electricity

- Semiconductor Electronics

- Electromagnetic Fields ${ }^{1}$

- Electromagnetic Technology ${ }^{1}$

\section{Chemical Science and Technology}

Laboratory

- Biotechnology

- Chemical Engineering ${ }^{1}$

- Chemical Kinetics and Thermodynamics

- Inorganic Analytical Research

- Organic Analytical Research

- Process Measurements

- Surface and Microanalysis Science

- Thermophysics ${ }^{2}$

\section{Physics Laboratory}

- Electron and Optical Physics

- Atomic Physics

- Molecular Physics

- Radiometric Physics

- Quantum Metrology

- Ionizing Radiation

- Time and Frequency ${ }^{1}$

- Quantum Physics ${ }^{1}$
Manufacturing Engineering Laboratory

- Precision Engineering

- Automated Production Technology

- Robot Systems

- Factory Automation

- Fabrication Technology

Materials Science and Engineering Laboratory

- Intelligent Processing of Materials

- Ceramics

- Materials Reliability ${ }^{1}$

- Polymers

- Metallurgy

- Reactor Radiation

Building and Fire Research Laboratory

- Structures

- Building Materials

- Building Environment

- Fire Science and Engineering

- Fire Measurement and Research

Computer Systems Laboratory

- Information Systems Engineering

- Systems and Software Technology

- Computer Security

- Systems and Network Architecture

- Advanced Systems

\section{Computing and Applied Mathematics}

Laboratory

- Applied and Computational Mathematics ${ }^{2}$

- Statistical Engineering ${ }^{2}$

- Scientific Computing Environments ${ }^{2}$

- Computer Services ${ }^{2}$

- Computer Systems and Communications ${ }^{2}$

- Information Systems

\footnotetext{
${ }^{1}$ At Boulder, CO 80303.

${ }^{2}$ Some elements at Boulder, CO 80303.
} 
Speed of Sound Data and Related Models for Mixtures of Natural Gas Constituents

B.A. Younglove

N.V. Frederick

R.D. McCarty

Thermophysics Division

Chemical Science and Technology Laboratory

National Institute of Standards and Technology

Boulder, Colorado 80303-3328

Sponsored by

The Gas Research Institute

Physical Sciences Department

January 1993

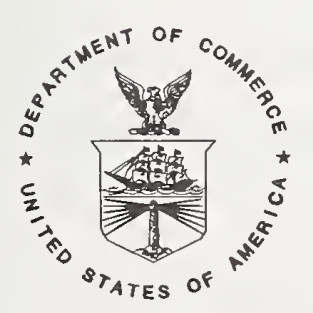

U.S. DEPARTMENT OF COMMERCE, Barbara Hackman Franklin, Secretary TECHNOLOGY ADMINISTRATION, Robert M. White, Under Secretary for Technology NATIONAL INSTITUTE OF STANDARDS AND TECHNOLOGY, John W. Lyons, Director 
National Institute of Standards and Technology

Natl. Inst. Stand. Technol., Mono. 178, 97 pages (Jan. 1993)

CODEN:NIMOEZ

\section{U.S. GOVERNMENT PRINTING OFFICE \\ WASHINGTON: 1993}

For sale by the Superintendent of Documents, U.S. Government Printing Office, Washington, DC 20402-9325 


\section{CONTENTS}

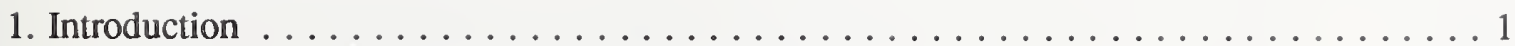

2. Experimental Method, Procedures, and Uncertainties $\ldots \ldots \ldots \ldots \ldots$

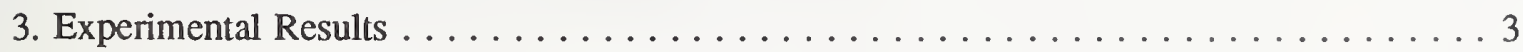

4. Predictive Models

4.1 NIST Model, NGAS .......................

4.2 AGA 8 Model $\ldots \ldots \ldots \ldots \ldots \ldots \ldots \ldots \ldots \ldots \ldots \ldots \ldots \ldots \ldots \ldots$

4.3 Johnson Model, Mass Flow Rate . . . . . . . . . . . . . . . 6

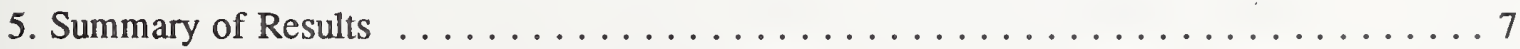

6. References $\ldots \ldots \ldots \ldots \ldots \ldots \ldots \ldots \ldots \ldots \ldots \ldots \ldots \ldots \ldots \ldots$

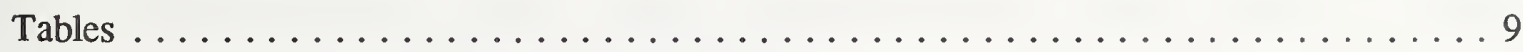

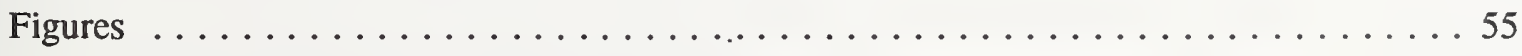




\section{List of Tables}

$\underline{\text { Page }}$

Table 1. Compositions in mole fraction for binary and multicomponent mixtures. . . . . 9

Table 2. Speed of sound data for the mixture $0.94985 \mathrm{CH}_{4}+0.05015 \mathrm{C}_{2} \mathrm{H}_{6} \cdot \ldots \ldots$

Table 3. Speed of sound data for the mixture $0.84992 \mathrm{CH}_{4}+0.15008 \mathrm{C}_{2} \mathrm{H}_{6} . \ldots \ldots 12$

Table 4. Speed of sound data for the mixture $0.68526 \mathrm{CH}_{4}+0.31474 \mathrm{C}_{2} \mathrm{H}_{6} . \ldots \ldots$

Table 5. Speed of sound data for the mixture $0.50217 \mathrm{CH}_{4}+0.49783 \mathrm{C}_{2} \mathrm{H}_{6} \cdot \ldots \ldots$

Table 6. Speed of sound data for the mixture $0.34524 \mathrm{CH}_{4}+0.65476 \mathrm{C}_{2} \mathrm{H}_{6} . \ldots \ldots$

Table 7. Speed of sound data for the mixture $0.90016 \mathrm{CH}_{4}+0.09984 \mathrm{C}_{3} \mathrm{H}_{8} . \ldots 21$

Table 8. Speed of sound data for the mixture $0.95114 \mathrm{CH}_{4}+0.04886 \mathrm{~N}_{2}$. $\ldots \ldots 23$

Table 9. Speed of sound data for the mixture $0.85130 \mathrm{CH}_{4}+0.14870 \mathrm{~N}_{2} . \ldots 26$

Table 10. Speed of sound data for the mixture $0.71373 \mathrm{CH}_{4}+0.28627 \mathrm{~N}_{2} . \ldots 28$

Table 11. Speed of sound data for the mixture $0.94979 \mathrm{CH}_{4}+0.05021 \mathrm{CO}_{2} . \ldots \ldots 31$

Table 12. Speed of sound data for the mixture $0.85026 \mathrm{CH}_{4}+0.14974 \mathrm{CO}_{2}$. $\ldots \ldots 34$

Table 13. Speed of sound data for the mixture $0.69944 \mathrm{CH}_{4}+0.30056 \mathrm{CO}_{2} . \ldots \ldots 36$

Table 14. Speed of sound data for the mixture $0.49593 \mathrm{~N}_{2}+0.50407 \mathrm{CO}_{2}$. $\ldots \ldots 39$

Table 15 . Speed of sound data for the Gulf Coast mixture. . . . . . . . . . . 41

Table 16. Speed of sound data for the Amarillo mixture. $\ldots \ldots \ldots \ldots \ldots \ldots$

Table 17. Speed of sound data for the Statoil dry gas mixture. . . . . . . . . . 47

Table 18. Speed of sound data for the Statoil Statvordgass mixture. . . . . . . . . 50

Table 19. Coefficients for NGAS, Equation $(3) \ldots \ldots \ldots \ldots \ldots \ldots \ldots \ldots \ldots \ldots$ 


\section{List of Figures}

$\underline{\text { Page }}$

Figure 1. Deviations of speed of sound computed by AGA 8 and NGAS from the experimental values for the pure methane data of Sivaraman and Gammon [8].

Figure 2. Deviations of speed of sound computed by AGA 8 and NGAS from the NIST experimental values for pure methane.

Figure 3. Experimental speed of sound for the

binary mixture methane 0.95 - ethane 0.05 .

Figure 4. Experimental speed of sound for the

binary mixture methane 0.85 - ethane 0.15 .

Figure 5. Experimental speed of sound for the

binary mixture methane 0.69 - ethane 0.31 .

Figure 6. Experimental speed of sound for the

binary mixture methane 0.50 - ethane 0.50 .

Figure 7. Experimental speed of sound for the

binary mixture methane 0.35 - ethane $0.65 . \ldots \ldots \ldots \ldots$

Figure 8. Experimental speed of sound for the

binary mixture methane 0.90 - propane 0.10 .

Figure 9. Experimental speed of sound for the

binary mixture methane 0.95 - nitrogen 0.05 .

Figure 10. Experimental speed of sound for the binary methane 0.85 - nitrogen 0.15 .

Figure 11. Experimental speed of sound for the

binary mixture methane 0.71 - nitrogen 0.29 .

Figure 12. Experimental speed of sound for the

binary mixture methane 0.95 - carbon dioxide 0.05 .

Figure 13. Experimental speed of sound for the

binary mixture methane 0.85 - carbon dioxide 0.15 .

Figure 14. Experimental speed of sound for the

binary mixture methane 0.70 - carbon dioxide 0.30 . 
Figure 15. Experimental speed of sound for the binary mixture nitrogen 0.50 - carbon dioxide 0.50 .

Figure 16. Experimental speed of sound for the Gulf Coast mixture.

Figure 17. Experimental speed of sound for the Amarillo mixture.

Figure 18. Experimental speed of sound for the Amarillo mixture. Comparing the $300 \mathrm{~K}$ run to the high pressure isotherm at $298 \mathrm{~K}$.

Figure 19. Experimental speed of sound for the Statoil dry gas mixture. $\ldots \ldots \ldots \ldots \ldots 6$

Figure 20. Experimental speed of sound for the Statoil Statvordgass mixture. . . . . . 66

Figure 21. Deviations of speed of sound computed by AGA 8 and NGAS from the experimental values for the binary mixture, methane 0.95 - ethane 0.05 .

Figure 22. Deviations of speed of sound computed by AGA 8 and NGAS from the experimental values for the binary mixture, methane 0.85 - ethane 0.15 .

Figure 23. Deviations of speed of sound computed by AGA 8 and NGAS from the experimental values for the binary mixture, methane 0.69 - ethane 0.31 .

Figure 24. Deviations of speed of sound computed by AGA 8 from the experimental values for the binary mixture methane 0.50 ethane 0.50 . Note: this composition is outside the range of AGA 8 and NGAS.

Figure 25. Deviations of speed of sound computed by AGA 8 from the experimental values for the binary mixture, methane 0.35 ethane 0.65 . Note: this composition is outside the range of AGA 8 and NGAS.

Figure 26. Deviations of speed of sound computed by AGA 8 and NGAS from the experimental values for the binary mixture, methane 0.90 - propane 0.10 . Note: this composition is outside the range of AGA 8 and NGAS.

Figure 27. Deviations of speed of sound computed by AGA 8 and NGAS from the experimental values for the binary mixture, methane 0.95 - nitrogen 0.05 .

Figure 28. Deviations of speed of sound computed by AGA 8 from the experimental values for the binary mixture, methane 0.85 nitrogen 0.15 . Note: this composition is outside the range of NGAS. 
Figure 29. Deviations of speed of sound computed by AGA 8 from the experimental values for the binary mixture, methane 0.71 -

nitrogen 0.29 . Note: this composition is outside the range of NGAS.

Figure 30. Deviations of speed of sound computed by AGA 8 and NGAS

from the experimental values for the binary mixture,

methane 0.95 - carbon dioxide $0.05 . \ldots \ldots \ldots \ldots \ldots \ldots \ldots$

Figure 31. Deviations of speed of sound computed by AGA 8 and NGAS from

the experimental values for the binary mixture, methane 0.85 -

carbon dioxide 0.15 . Note: this composition is outside the

range of NGAS.

Figure 32. Deviations of speed of sound computed by AGA 8 from the

experimental values for the binary mixture, methane 0.70 -

carbon dioxide 0.30 . Note: this composition is outside

the range of NGAS.

Figure 33. Deviations of speed of sound computed by AGA 8 from the experimental values for the binary mixture, nitrogen 0.50 -

carbon dioxide 0.50 . Note: this composition is outside the range

of AGA 8 and NGAS.

Figure 34. Deviations of speed of sound computed by AGA 8 and NGAS

from the experimental values for the Gulf Coast mixture.

Figure 35. Deviations of speed of sound computed by AGA 8 and NGAS

from the experimental values for the Amarillo mixture.

Figure 36. Deviations of speed of sound computed by AGA 8 and NGAS

from the experimental values for the Statoil dry gas mixture.

Figure 37. Deviations of speed of sound computed by AGA 8 and NGAS

from the experimental values for the Statoil Statvordgass mixture.

Figure 38. Deviations of the densities computed by NGAS from those computed using AGA 8 for the Gulf Coast mixture. . . . . . . . . . . . 84

Figure 39. Deviations of the densities computed by NGAS from those computed using AGA 8 for the Amarillo mixture.

Figure 40. Deviations of the densities computed by NGAS from those computed using AGA 8 for the Statoil dry gas mixture.

Figure 41. Deviations of the densities computed by NGAS from those computed using AGA 8 for the Statoil Statvordgass mixture. 
Figure 42. Deviations of mass flow computed by the Johnson equation from that computed by NGAS for methane 0.95 - ethane $0.05 . \ldots \ldots \ldots \ldots \ldots \ldots \ldots \ldots$. . . . . . . . . . .

Figure 43. Deviations of mass flow computed by the Johnson equation from that computed by NGAS for methane 0.70 - ethane 0.30 .

Figure 44. Deviations of mass flow computed by the Johnson equation from that computed by NGAS for methane 0.95 - carbon dioxide 0.05 .

Figure 45. Deviations of mass flow computed by the Johnson equation from that computed by NGAS for methane 0.90 - carbon dioxide 0.10 .

Figure 46. Deviations of mass flow computed by the Johnson equation from that computed by NGAS for methane 0.95 - nitrogen 0.05 .

Figure 47. Deviations of mass flow computed by the Johnson equation from that computed by NGAS for methane 0.90 - nitrogen 0.10 .

Figure 48. Deviations of mass flow computed by the Johnson equation from that computed by NGAS for the Gulf Coast mixture.

Figure 49. Deviations of mass flow computed by the Johnson equation from that computed by NGAS for the Amarillo mixture. 
Speed of Sound Data and Related Models

for Mixtures of Natural Gas Constituents

B.A. Younglove, N.V. Frederick, and R.D. McCarty

\author{
Thermophysics Division \\ National Institute of Standards and Technology \\ 325 Broadway \\ Boulder, Colorado 80303-3328
}

\begin{abstract}
Sound speed data have been obtained for thirteen binary mixtures and four multicomponent mixtures of natural gas components using a cylindrical cavity. These data cover a temperature range from 250 to $350 \mathrm{~K}$ at pressures to $10 \mathrm{MPa}$. The uncertainty in the data is approximately 0.05 percent. The binary mixtures are primarily methane-rich, with ethane, nitrogen, carbon dioxide, or propane as the second component. The multicomponent mixtures are representative of commercially available compositions in the United States and Europe. The data were used to develop and test mathematical models for prediction of the sound speed of natural gas mixtures, within an average uncertainty of 0.1 percent, over the ranges of pressure, temperature, and composition that encompass the major region of custody transfer for natural gas. The research program was managed and sponsored by the Gas Research Institute's Physical Sciences Department.
\end{abstract}

Key words: binary mixtures; carbon dioxide; ethane; gas; isotherm; methane; multicomponent mixtures; natural gas; nitrogen; propane; sound speed

\title{
1. Introduction
}

This report summarizes the results of a project focusing on obtaining sound speed data for primarily binary mixtures of natural gas components. It represents the first comprehensive measurement

program of sound speed data for natural gas mixtures. The program was managed and sponsored by the Gas Research Institute (GRI), Physical Sciences Department.

Orifice plates and turbine meters are widely used to determine the mass flow rate of natural gas. Calibration of a flow meter can be accurately accomplished by measuring the mass flow rate with a sonic nozzle placed in series with the meter. The sonic nozzle is operated at maximum flow rate which is obtained at the speed of sound of the system, at a given temperature, pressure, and composition. 
We can compute the mass flow rate $[1,2]$ using

$$
\mathbf{m}=\mathbf{C A} \rho \mathbf{W}
$$

where $\mathbf{m}$ is the mass flow rate, $\mathbf{A}$ is the cross-sectional area of the nozzle at the throat, $\rho$ is the density, $\mathbf{W}$ is the sound speed, and $\mathbf{C}$ is a calibration constant for the nozzle and depends on geometrical imperfections and energy losses of the nozzle. The ideal value for $\mathbf{C}$ is unity, for a lossless system. The temperature, pressure, and composition are used to compute the density and sound speed from an appropriate equation of state.

In the present study, sound speed data have been obtained for mixtures of natural gas components. The major objective of this work has been to provide the natural gas industry with experimental data that can then be correlated by an equation of state:

$$
\mathbf{W}=\mathbf{W}\left(\mathbf{P}, \mathbf{T}, \mathbf{x}_{\mathbf{i}}\right)
$$

Speed of sound data on methane and its binary mixtures are used in the development of the model. Data have been obtained for thirteen binary mixtures. These are primarily methane-rich with either propane, carbon dioxide, or nitrogen as the second component. Sound speed results for methane have been measured at $273.15 \mathrm{~K}$ and compared with Gammon and Douslin's wide-range data [3], used in the development of the model. The data on four multicomponent mixtures (Gulf Coast, Amarillo, Statoil* dry gas, and Statoil" Statvordgass) have been obtained to test the predictions of speed of sound models. All of these mixtures were prepared gravimetrically using a high-precision balance. Measurements were taken on five isotherms, at 250,275, 300, 325, and $350 \mathrm{~K}$, at pressures to $10 \mathrm{MPa}$. This range of temperature and pressure encompasses the major region involved in custody transfer. All of the experimental measurements at NIST were made using a cylindrical resonant cavity with a fixed path length. The data of Gammon and Douslin [3] for pure methane were obtained using an interferometer of variable path length.

This monograph reports all of the new experimental sound speed data obtained in this study. Details of a new model developed at NIST, called NGAS [4], for predictions of sound speed and density of natural gas mixtures are presented. Comparisons of this model, the AGA 8 (American Gas Association) model [5,6], and the traditional approach of Johnson [7] are made with the comprehensive set of experimental data for natural gas mixtures and the

"Certain commercial materials, equipment, or instruments are identified in this paper in order to adequately specify the experimental results. Such identification does not imply endorsement by the National Institute of Standards and Technology, nor does it imply that the materials or equipment that are identified are necessarily the best available for the purpose. 
pure methane data [8].

\section{Experimental Method, Procedures, and Uncertainties}

The details of the experimental procedure are given in [9]. The measurements were made using a cylindrical resonant cavity operating at frequencies between 10 and $70 \mathrm{kHz}$. Longitudinal resonances were measured, and the frequencies were corrected for shifts arising from viscous losses at the walls and for thermal conduction losses at the walls and end surfaces. Uncertainty in sound speed measurements is less than 0.05 percent. Temperatures were measured on the IPTS- 68 temperature scale with a capsule platinum resistance thermometer. The sample temperature was regulated within $3 \mathrm{mK}$. The total uncertainty in the temperature measurement is approximately $0.02 \mathrm{~K}$ at 250 $\mathrm{K}$ and $0.03 \mathrm{~K}$ at $350 \mathrm{~K}$. These temperatures may be converted to the ITS-90 scale using procedures described by H. Preston-Thomas [10]. Pressures were measured with a high-quality quartz-spiral bourdon gauge. The uncertainty in pressure measurement is estimated to be $0.001 \mathrm{MPa}$. The samples were prepared in clean aluminum cylinders which were heated and pumped to a high vacuum. Mixtures were prepared by weighing using a 25-kg, highprecision double pan balance. The maximum uncertainty in mole fraction is estimated to be 0.006 percent.

\section{Experimental Results}

Table 1 gives the compositions, in mole percent, of the thirteen binary and four multicomponent mixtures. The sound speed data are given in tables 2 through 18. The table entries are experimental sound speed in meters per second, temperature in kelvins, and pressure in megapascals or in pounds per square inch absolute. The experimental sound speed data are seen in figures 1 through 20, as a function of pressure along isotherms.

\section{Predictive Models \\ 4.1 NIST Model, NGAS}

The functional form of the NIST model, NGAS [4], is given below in eq (3). $A_{r}$ is the real gas contribution to the Helmholtz energy. The pressure and temperature ranges of the equation of state are 0 to $10 \mathrm{MPa}$ and $250 \mathrm{~K}$ to $350 \mathrm{~K}$. 


$$
\begin{aligned}
A_{r} & =R T z \sum_{i j}\left[N_{1}+N_{2} t_{i j}^{1.5}(G 3)_{i j}+N_{3} t_{i j}^{2.5}+N_{4} t_{i j}^{2}+N_{5} t_{i j}^{3.5}(G 3)_{i j}\right] x_{i} x_{j} \\
& +\left(N_{6}+N_{7} \tau^{0.5}+N_{8} \tau^{2}+N_{9} \tau^{2.5}+N_{16} / \tau^{0.5} N_{17} \tau^{1.5}\right) R T z^{2}(G 3) \\
& +\left(N_{10} \tau^{0.5}+N_{11} \tau+N_{12} \tau^{1.5}+N_{18}\right) R T z^{3}(G 4) \\
& +\left(N_{14} \tau^{3}+N_{22} \tau^{3.5}+N_{23} \tau^{5.5}\right) R T z^{2}(G 5) \mathrm{e}^{-z^{2}} \\
& +\left(N_{13} z^{2} \tau+N_{24} \tau^{3} \mathrm{e}^{-z^{2}}\right) R T z^{3}(G 6) \\
& +\left(N_{19} z^{4}+N_{15} \tau^{3} z^{2} e^{-z^{2}}+N_{26} \tau^{22} e^{-z^{2}}\right) R T z^{2}(G 7) \\
& +\left(N_{20} z^{4} \tau^{2}+N_{27} \tau^{18} e^{-z^{4}}\right) R T z^{3}(G 8) \\
& +\left[N_{21} z^{4} \tau^{2}+\left(N_{28} \tau^{11}+N_{29} \tau^{23}\right) \mathrm{e}^{-z^{4}}\right] R T z^{4}(G 9) \\
& +N_{30} z^{5} \tau^{18} e^{-z^{4}}(G 10)+N_{25} z^{10} \tau^{5.5} e^{-z^{2}}(G 11)
\end{aligned}
$$

where

$$
\begin{array}{ll}
\mathbf{t}_{i j} & =\mathbf{e}_{i j} / T, \\
\mathbf{e}_{i j} & =\mathbf{w}_{i j}\left(\varepsilon_{i} \varepsilon_{j}\right)^{0.5} \\
\mathbf{z} & =\rho \sigma^{3} \\
\sigma^{6} & =\sum_{i} \sum_{j} \mathbf{x}_{i} \mathbf{x}_{j} \sigma_{i j}^{6}, \\
\sigma_{i j}^{3} & =v_{i j}\left(\sigma_{i}^{3} \sigma_{j}^{3}\right)^{0.5} \\
\tau & =\varepsilon / T, \\
\varepsilon & =\sigma^{-6} \sum_{i} \sum_{j} \mathbf{x}_{i} \mathbf{x}_{j} \sigma_{i j}^{6} \varepsilon_{i j}, \\
\varepsilon_{i j} & =u_{i j}\left(\varepsilon_{i} \varepsilon_{j}\right)^{0.5}, \\
(G k) & =\sum_{i j} \sum_{j} \mathbf{x}_{i} \mathbf{x}_{j}(G k)_{i j} k=1,2, \ldots 11, \\
(G k)_{i j} & =\left(G k_{i}+G k_{j}\right) / 2 .
\end{array}
$$

$\mathbf{A}_{r}$ is the real gas contribution to the Helmholtz energy. $R=8.31434$ is the gas constant, $T$ is the temperature in kelvins, and $\rho$ is the density in moles per liter. The $\mathbf{N}_{\mathbf{i}}$ for eq (3) were obtained by a least squares fit to the experimental sound speed data of Gammon and Douslin [3] and of Sivaraman and Gammon [8] simultaneously with the PVT results of Friend and Ely [11], and are given in table 19. The allowable pure 
components are the same as for the AGA Report No. 8 equation of state $[5,6]$, with the exclusion of $\mathrm{H}_{2} \mathrm{~S}$ and $\mathrm{H}_{2} \mathrm{O}$. See the listing in table 19. The $\sigma^{3}, \varepsilon_{1}, \mathbf{G k}_{\mathbf{l}}, \mathbf{u}_{i j}, \mathbf{v}_{i j}$, and $\mathbf{w}_{i j}$ were obtained by least squares fits to the binary sound speed data simultaneously with PVT results from [12,13] and are given in table 19. The total Helmholtz energy is given by

$$
\mathbf{A}=\mathbf{A}_{\mathbf{r}}+\mathbf{A}^{0}
$$

where $\mathbf{A}^{0}$ is the ideal gas contribution to the Helmholtz energy. The pressure derived from this expression is

$$
\mathbf{p}=\rho^{2}(\partial \mathbf{A} / \partial \rho)_{\mathrm{T}}
$$

The Helmholtz energy for the ideal gas is

$$
\mathbf{A}^{\circ}=\mathbf{H}^{\circ}-\mathbf{R T}-\mathbf{T S}^{\circ}
$$

where $\mathbf{H}^{\circ}$ and $\mathbf{S}^{\circ}$ are the ideal gas enthalpy and entropy, and $\mathrm{T}$ is the absolute temperature. To insure consistency, $\mathbf{H}^{\circ}$ and $\mathbf{S}^{\circ}$ from Starling [12] were used here, and the remainder of the thermodynamic properties were obtained from the appropriate derivatives of the Helmholtz energy from the new model reported here.

In addition to the restricted pressure and temperature ranges mentioned above, the equation of state is valid only for mixtures containing at least 60 percent methane, no more than 5 percent nitrogen or carbon dioxide, and no more than 1 percent total of $\mathbf{C}_{4}$ and above. The predicted speeds of sound from eq (3) have been compared to the experimental data. In figures $1,1 \mathrm{a}$, and $1 \mathrm{~b}$ deviations are shown on selected isotherms for the pure methane data of Gammon and Douslin [3] and of Sivaraman and Gammon [8]. Figure 2 shows the deviation for one isotherm of pure methane at $298.15 \mathrm{~K}$ taken with the cylindrical resonator. The deviation plots show that both models are generally within 0.1 percent of the experimental sound speeds from 223 to $348 \mathrm{~K}$. Comparisons to the binary and multicomponent data are given in figures 21 through 37 . These comparisons are made relative to the stated composition restrictions of the NGAS and AGA 8 equations of state. These deviation plots show that both eq (3) and AGA 8 predict sound speeds of the multicomponent gas mixtures within 0.1 percent for the mixtures that are within the equation limits, with the exception of the lowest temperature $(250 \mathrm{~K})$ for pressures above $5 \mathrm{MPa}$. In the low temperature, higher pressure regime, neither model agrees with the experimental data very well, but the deviation pattern of each model is different. Although the equations do not predict sound speeds to the desired 0.1 percent in this extreme temperature and pressure range, it is of little importance in the application to sonic metering since the pressure in the throat of the nozzle will always be less than $5 \mathrm{MPa}$ if the upstream pressure is within the specified range of the equation of state. A computer program using eq (3) has been developed to calculate the 
thermodynamic properties of natural gas. In addition to the standard thermodynamic properties, the program also calculates the mass flow rate and the critical flow factor for a sonic meter. The program requires pressure, temperature, and composition as input. Copies of the NGAS program may be obtained from the Gas Research Institute. Computer programs using the AGA Report No. 8 model are available through the American Gas Association or the Gas Research Institute.

\subsection{AGA 8 Model}

Recently, work sponsored by the Gas Research Institute and the American Gas Association at the Universities of Oklahoma and Idaho has produced an improved equation of state, called in this work the AGA 8 model $[5,6]$. The speed of sound data obtained under GRI's program and reported here were used in the development of the new AGA 8 model. This model also incorporates the speed of sound data for methane of Sivaraman and Gammon [8]. Other data used in its development include pure component compressibility data, compressibility data for certain binary mixtures, and GERG (Groupe Européen de Recherches Gazeriès) compressibility data [14].

Figures 21 through 37, mentioned in the previous section, also present limited deviations between values calculated from the AGA 8 model and measured sound speeds. Some comparisons are made outside of the stated limits of the AGA 8 model. The comparison between the NGAS model and the AGA 8 model provided by these deviation plots indicates very little difference between the two models except at the lower temperatures for pressures above $5 \mathrm{MPa}$. In this low-temperature higher pressure regime neither model agrees with the experimental data very well, but the deviation pattern of each model is different. The NGAS model is probably somewhat better in this difficult region. Figures 38 through 41 give a comparison of densities calculated by means of the two models at the pressure and temperature of some of the measured sound speeds. Since there are no measured densities for these pressures and temperatures, no conclusions as to the accuracy of either of these models may be drawn from the deviation plots. The comparison does, however, indicate some interesting behavior. The densities calculated from either model are quite similar except in the low temperature, higher pressure region where the two models do not perform well in the prediction of sound speed, and for the Statoil Statvordgass mixture where there is about a 0.2 percent offset between the two models. Another interesting feature of the density deviation plots is an abrupt change in the deviation pattern of some of the isotherms at a pressure of $1 \mathrm{MPa}$ for each of the mixtures, with the single exception of the Statoil dry gas mixture.

\subsection{Johnson Model, Mass Flow Rate}

The mass flow rates for a variety of compositions and plenum conditions have been calculated from the critical flow equations shown below and the equation of state presented here. A sonic nozzle operates at maximum mass flow. The mass flow speed at the throat of the nozzle is the speed of sound. This condition allows the 
calculation of the mass flow rate, $\mathbf{m}$, which is given in eq (1). Under the assumption that the fluid flow is one dimensional and that the entropy $S_{t}$ of the fluid in the nozzle throat is the same as the entropy $S_{p}$ of the fluid on the up-stream side or plenum of the nozzle,

$$
\begin{gathered}
S_{p}-S_{t}=0, \\
U_{t}^{2}=2\left(H_{p}-H_{t}\right),
\end{gathered}
$$

where $\mathrm{H}$ is the enthalpy of the fluid and $\mathrm{U}$ is the mass flow rate in the nozzle, and the flow in the nozzle is sonic,

$$
U_{t}^{2}=w_{t}^{2}
$$

The conditions described by eqs (1) and (17) through (19) provide the basis for computation of mass flow rates for the sonic nozzle. Figures 42 through 49 compare these flow rates with those predicted by Johnson [7].

The deviations illustrated by figures 42 through 49 indicate a degraded performance of the Johnson model at higher pressures and lower temperatures. The deviation plots also indicate that the Johnson model is less reliable for mixtures containing significant amounts of components other than methane.

\section{Summary of Results}

The speed of sound measurements are given in this report for thirteen binary mixtures and four multicomponent mixtures of natural gas components. The accuracy of the experimental speed of sound data is estimated to be within 0.05 percent. The experimental data and the deviations from the predictive models, NGAS and AGA 8, are given in the form of graphs and tables. Both NGAS and AGA 8 were developed using the speed of sound data contained in this report as part of their correlation database. The functional form of NGAS is presented. Deviation plots for NGAS and AGA 8 from the pure methane data of Sivaraman and Gammon are also given. A comparison of the densities as predicted by NGAS and AGA 8 is given for the four multicomponent mixtures. The mass flow rates as computed from NGAS are compared to those from the Johnson formulation [13].

Deviations of the experimental sound speed from the values computed using NGAS and AGA 8 are given for each isotherm. In some cases AGA 8 deviations are shown beyond the limits for AGA 8 to allow comparisons with NGAS. Generally the values of sound speed computed from the equations of state are within \pm 0.1 percent of the experimental data for the $273,300,325$, and $350 \mathrm{~K}$ isotherms. The largest deviations are seen for data taken at $250 \mathrm{~K}$.

This work was carried out at the National Institute of Standards and Technology under the sponsorship of the Gas Research Institute, Physical Sciences Department, Thermodynamics Program, Program Manager, Jeffrey L. Savidge. 
6. References

[1] Miller, R.W., "Flow Measurement Engineering Handbook," 2nd. ed., McGraw Hill (1989).

[2] Arnberg, B.T., "Review of Critical Flow Meters for Gas FLow Measurements," J. Basic Eng. D84, 447-460 (1962).

[3] Gammon, B.E., and Douslin, D.R., "The velocity of sound and heat capacity in methane from near-critical to subcritical conditions and equation-of-state implications," J. Chem. Phys. 64, 203-218, (1976).

[4] McCarty, R.D., "A Model for the Speed of Sound of Natural Gas Mixtures," Proceedings of the International Symposium on Fluid Flow Measurement, June 6-8, 1990, published by American Gas Association, Arlington VA, Catalog No. XQ9010, p. 155-169 (1990).

[5] Starling, K.E., and Savidge, J.L., "Compressibility Factors of Natural Gas and Other Related Hydrocarbon Gases," American Gas Association Transmission Measurement Committee Report No. 8, American Gas Association, Arlington VA, Catalog No. XQ9212, (1992).

[6] Starling, K.E., and Savidge, J.L., "GRI High Accuracy Natural gas Equation of State for Gas Measurement Applications," Annual Report to American Gas Association Transmission Measurement Committee, Supercompressibility Factor Steering Committee, GRI-91/0184, (1991).

[7] Johnson, R.C., "Calculations of the Flow of Natural Gas Through Critical Flow Nozzles," Transactions of the ASME, p. 580 (September 1970).

[8] Sivaraman, A., and Gammon, B.E., "Speed-of-Sound Measurements in Natural Gas Fluids," Gas Research Institute Report 86-0043 (1986).

[9] Younglove, B.A., and Frederick, N.V., "Sound Speed Measurements on Gas Mixtures of Natural Gas Components Using a Cylindrical Resonator," Intl. J. Thermophys., 11, 897-909 (1990).

[10] Preston-Thomas, H., "The International Temperature Scale of 1990 (ITS-90)," Metrologia, 27, 3-10 (1990).

[11] Friend, D.G., and Ely, J.F., "Thermophysical Properties of Methane," J. Phys. Chem. Ref. Data, 18, No. 2, 583-638 (1986).

[12] Starling, K.E., Mannan, M., Savidge, J.L., Sadasivan S., Reid, T.B. Jr., Gangadhar, K., and Drass M.A., Appendix C, Final Report, October 1984 - September 1987, Prepared for Gas Research Institute, Contract No. 5084-260-1010 (September 1987), University of Oklahoma, Norman, Oklahoma.

[13] Starling, K.E., and Fitz, C., GRI computer program, private communication, University of Oklahoma, Norman, Oklahoma.

[14] Jaeschke, M., Audibert, S., van Caneghem, P., Humpreys, A.E., Jansen van Rosmalen, R., and Pellei, Q., "High Accuracy Compressibility Factor Calculation for Natural Gases and Similar Mixtures by Use of a Truncated Virial Equation," GERG Technical Monograph 2 (1988). 
Table 1. Compositions in mole fraction for binary and multicomponent mixtures.

Compositions in mole fractions for the binary mixtures.

\begin{tabular}{||l||}
\hline 0.84992 methane -0.15008 ethane \\
\hline 0.68526 methane -0.31474 ethane \\
\hline 0.50217 methane -0.49783 ethane \\
\hline 0.34524 methane -0.65476 ethane \\
\hline 0.90016 methane -0.09984 propane \\
\hline 0.95114 methane -0.04886 nitrogen \\
\hline 0.85130 methane -0.14870 nitrogen \\
\hline 0.71373 methane -0.28627 nitrogen \\
\hline 0.94979 methane -0.05021 carbon dioxide \\
\hline 0.85026 methane -0.14974 carbon dioxide \\
\hline 0.69944 methane -0.30056 carbon dioxide \\
\hline 0.49593 nitrogen -0.50407 carbon dioxide \\
\hline
\end{tabular}

Compositions in mole fractions for the multicomponent mixtures.

\begin{tabular}{|c|c|c|c|c|}
\hline & Gulf Coast & Amarillo & $\begin{array}{l}\text { Statoil } \\
\text { Dry Gas }\end{array}$ & $\begin{array}{l}\text { Statoil } \\
\text { Statvordgas }\end{array}$ \\
\hline methane & 0.96561 & 0.90708 & 0.83980 & 0.74348 \\
\hline ethane & 0.01829 & 0.04491 & 0.13475 & 0.12005 \\
\hline propane & 0.00410 & 0.00815 & 0.00943 & 0.08251 \\
\hline normal butane & 0.00098 & 0.00141 & 0.00067 & 0.03026 \\
\hline isobutane & 0.00098 & 0.00106 & 0.00040 & -- \\
\hline normal pentane & 0.00032 & 0.00065 & 0.00008 & 0.00575 \\
\hline isopentane & 0.00046 & 0.00027 & 0.00013 & -- \\
\hline normal hexane & 0.00067 & 0.00034 & -- & 0.00230 \\
\hline nitrogen & 0.00262 & 0.03113 & 0.00718 & 0.00537 \\
\hline carbon dioxide & 0.00597 & 0.00500 & 0.00756 & 0.01028 \\
\hline
\end{tabular}


Table 2. Speed of sound data for the mixture $0.94985 \mathrm{CH}_{4}+0.05015 \mathrm{C}_{2} \mathrm{H}_{6}$.

\begin{tabular}{|c|c|c|c|}
\hline $\begin{array}{l}W_{\exp } \\
\mathrm{m} / \mathrm{s}\end{array}$ & $\begin{array}{l}\text { TEMP } \\
\mathbf{K}\end{array}$ & $\begin{array}{l}\text { PRES } \\
\mathrm{MPa}\end{array}$ & $\begin{array}{l}\text { PRES } \\
\text { psia }\end{array}$ \\
\hline 377.77 & 250.000 & 9.864 & 1430.6 \\
\hline 377.11 & 250.000 & 9.800 & 1421.3 \\
\hline 370.94 & 250.001 & 9.050 & 1312.5 \\
\hline 367.57 & 250.000 & 8.401 & 1218.4 \\
\hline 365.72 & 250.000 & 7.685 & 1114.6 \\
\hline 365.60 & 250.000 & 7.520 & 1090.7 \\
\hline 365.65 & 250.000 & 6.913 & 1002.6 \\
\hline 366.96 & 250.000 & 6.193 & 898.2 \\
\hline 369.26 & 250.000 & 5.496 & 797.1 \\
\hline 373.92 & 250.000 & 4.493 & 651.7 \\
\hline 380.02 & 250.000 & 3.428 & 497.1 \\
\hline 386.97 & 250.000 & 2.356 & 341.7 \\
\hline 394.64 & 250.000 & 1.253 & 181.7 \\
\hline 397.00 & 250.000 & 0.915 & 132.7 \\
\hline 397.97 & 250.000 & 0.777 & 112.7 \\
\hline 407.39 & 275.000 & 10.588 & 1535.7 \\
\hline 404.48 & 275.000 & 10.059 & 1458.9 \\
\hline 400.79 & 275.000 & 9.152 & 1327.3 \\
\hline 398.69 & 275.000 & 8.297 & 1203.4 \\
\hline 397.85 & 275.000 & 7.522 & 1091.0 \\
\hline 398.01 & 275.000 & 6.677 & 968.4 \\
\hline 399.27 & 275.000 & 5.735 & 831.8 \\
\hline 401.26 & 275.000 & 4.872 & 706.6 \\
\hline 404.78 & 275.000 & 3.754 & 544.5 \\
\hline 408.25 & 275.000 & 2.868 & 416.0 \\
\hline 408.62 & 275.000 & 2.795 & 405.4 \\
\hline 411.87 & 275.000 & 2.041 & 296.0 \\
\hline 417.03 & 275.000 & 0.960 & 139.2 \\
\hline 418.60 & 275.000 & 0.646 & 93.8 \\
\hline 431.00 & 300.002 & 10.437 & 1513.8 \\
\hline 429.28 & 300.000 & 9.952 & 1443.4 \\
\hline 426.69 & 300.000 & 8.995 & 1304.6 \\
\hline 424.96 & 300.000 & 7.952 & 1153.3 \\
\hline 424.31 & 300.000 & 6.954 & 1008.6 \\
\hline 424.77 & 300.000 & 5.650 & 819.5 \\
\hline 426.09 & 300.000 & 4.603 & 667.6 \\
\hline 428.22 & 300.000 & 3.495 & 506.8 \\
\hline 431.15 & 300.000 & 2.360 & 342.2 \\
\hline 434.27 & 300.000 & 1.341 & 194.5 \\
\hline 435.38 & 300.000 & 1.019 & 147.8 \\
\hline 436.70 & 300.000 & 0.624 & 90.5 \\
\hline
\end{tabular}


Table 2. Speed of sound data for the mixture

$0.94985 \mathrm{CH}_{4}+0.05015 \mathrm{C}_{2} \mathrm{H}_{6}$ (continued).

\begin{tabular}{|c|c|c|c|}
\hline $\begin{array}{l}W_{\text {exp }} \\
\mathrm{m} / \mathrm{s}\end{array}$ & $\begin{array}{l}\text { TEMP } \\
\mathrm{K}\end{array}$ & $\begin{array}{l}\text { PRES } \\
\text { MPa }\end{array}$ & $\begin{array}{l}\text { PRES } \\
\text { psia }\end{array}$ \\
\hline 453.33 & 325.000 & 10.402 & 1508.7 \\
\hline 451.77 & 325.002 & 9.892 & 1434.7 \\
\hline 449.61 & 325.000 & 8.983 & 1302.9 \\
\hline 448.22 & 325.000 & 8.281 & 1201.1 \\
\hline 447.43 & 325.000 & 7.590 & 1100.9 \\
\hline 446.42 & 324.999 & 6.205 & 899.9 \\
\hline 446.51 & 325.002 & 5.521 & 800.8 \\
\hline 446.80 & 325.000 & 4.821 & 699.3 \\
\hline 447.37 & 325.000 & 4.124 & 598.1 \\
\hline 448.05 & 324.999 & 3.447 & 500.0 \\
\hline 449.15 & 325.000 & 2.668 & 387.0 \\
\hline 450.20 & 325.000 & 2.058 & 298.6 \\
\hline 451.55 & 325.000 & 1.411 & 204.6 \\
\hline 452.01 & 325.001 & 1.247 & 180.9 \\
\hline 452.42 & 325.000 & 1.086 & 157.5 \\
\hline 452.75 & 325.001 & 0.937 & 136.0 \\
\hline 453.06 & 325.001 & 0.804 & 116.6 \\
\hline 453.39 & 325.000 & 0.660 & 95.8 \\
\hline 453.78 & 325.000 & 0.496 & 71.9 \\
\hline 473.74 & 350.000 & 10.521 & 1525.9 \\
\hline 472.79 & 350.000 & 10.166 & 1474.4 \\
\hline 472.74 & 350.000 & 10.164 & 1474.1 \\
\hline 470.67 & 350.000 & 9.350 & 1356.1 \\
\hline 468.95 & 350.000 & 8.528 & 1236.9 \\
\hline 468.77 & 350.000 & 8.350 & 1211.0 \\
\hline 467.57 & 350.000 & 7.558 & 1096.1 \\
\hline 466.65 & 350.000 & 6.729 & 975.9 \\
\hline 466.03 & 350.000 & 5.931 & 860.3 \\
\hline 465.73 & 350.000 & 5.162 & 748.7 \\
\hline 465.71 & 350.000 & 4.369 & 633.7 \\
\hline 465.94 & 350.000 & 3.582 & 519.6 \\
\hline 466.39 & 350.000 & 2.819 & 408.9 \\
\hline 466.75 & 350.000 & 2.440 & 353.9 \\
\hline 467.13 & 350.000 & 2.068 & 300.0 \\
\hline 467.92 & 350.000 & 1.344 & 194.9 \\
\hline 468.12 & 350.000 & 1.203 & 174.4 \\
\hline 468.28 & 350.000 & 1.081 & 156.8 \\
\hline 468.69 & 350.000 & 0.793 & 115.1 \\
\hline 468.99 & 350.000 & 0.553 & 80.2 \\
\hline
\end{tabular}


Table 3. Speed of sound data for the mixture $0.84992 \mathrm{CH}_{4}+0.15008 \mathrm{C}_{2} \mathrm{H}_{6}$.

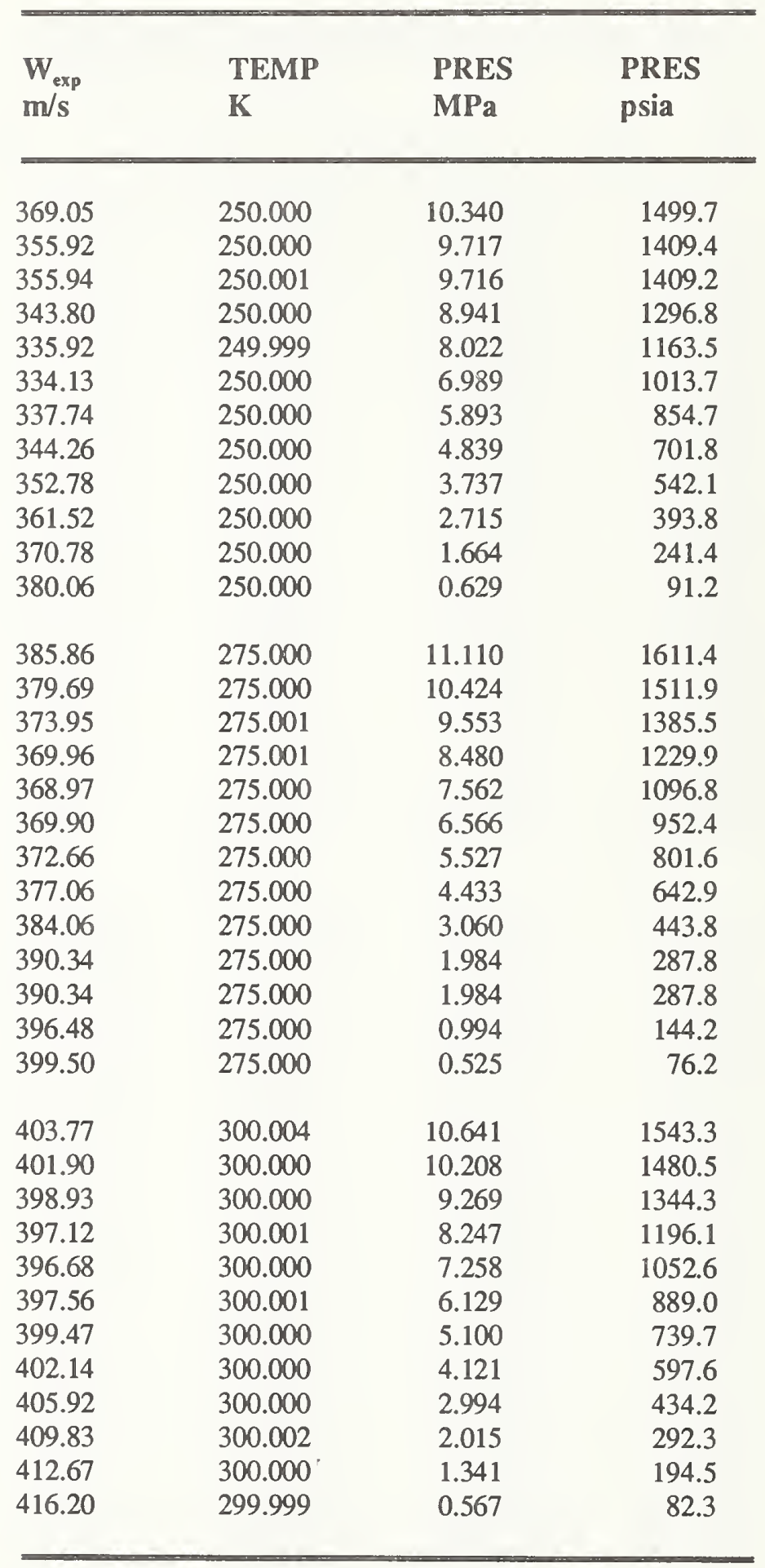


Table 3. Speed of sound data for the mixture $0.84992 \mathrm{CH}_{4}+0.15008 \mathrm{C}_{2} \mathrm{H}_{6}$ (continued).

\begin{tabular}{|c|c|c|c|}
\hline $\begin{array}{l}W_{\exp } \\
\mathrm{m} / \mathrm{s}\end{array}$ & $\begin{array}{l}\text { TEMP } \\
\mathbf{K}\end{array}$ & $\begin{array}{l}\text { PRES } \\
\mathrm{MPa}\end{array}$ & $\begin{array}{l}\text { PRES } \\
\text { psia }\end{array}$ \\
\hline 431.85 & 325.000 & 0.661 & 95.8 \\
\hline 431.71 & 325.000 & 0.682 & 98.9 \\
\hline 431.25 & 325.000 & 0.811 & 117.7 \\
\hline 430.76 & 325.000 & 0.968 & 140.3 \\
\hline 430.30 & 325.000 & 1.105 & 160.3 \\
\hline 429.84 & 325.000 & 1.238 & 179.5 \\
\hline 429.43 & 325.000 & 1.361 & 197.3 \\
\hline 421.24 & 325.000 & 4.833 & 701.0 \\
\hline 420.58 & 325.000 & 5.506 & 798.6 \\
\hline 420.02 & 325.000 & 6.283 & 911.3 \\
\hline 419.73 & 325.000 & 7.040 & 1021.0 \\
\hline 420.13 & 325.000 & 7.805 & 1132.0 \\
\hline 421.04 & 325.000 & 8.636 & 1252.5 \\
\hline 422.27 & 325.000 & 9.397 & 1362.9 \\
\hline 424.26 & 325.000 & 10.145 & 1471.5 \\
\hline 425.17 & 325.000 & 10.450 & 1515.6 \\
\hline 445.49 & 350.000 & 10.457 & 1516.6 \\
\hline 444.05 & 350.000 & 9.861 & 1430.2 \\
\hline 441.61 & 350.000 & 8.549 & 1240.0 \\
\hline 440.47 & 350.000 & 7.586 & 1100.3 \\
\hline 440.49 & 350.000 & 7.585 & 1100.0 \\
\hline 439.93 & 350.000 & 6.553 & 950.4 \\
\hline 439.87 & 350.000 & 6.370 & 923.9 \\
\hline 439.98 & 350.000 & 5.523 & 801.0 \\
\hline 440.55 & 350.000 & 4.497 & 652.2 \\
\hline 441.70 & 350.000 & 3.416 & 495.4 \\
\hline 443.17 & 350.000 & 2.430 & 352.5 \\
\hline 445.21 & 350.000 & 1.361 & 197.4 \\
\hline 446.04 & 350.000 & 0.961 & 139.4 \\
\hline 446.39 & 350.000 & 0.805 & 116.8 \\
\hline
\end{tabular}


Table 4. Speed of sound data for the mixture $0.68526 \mathrm{CH}_{4}+0.31474 \mathrm{C}_{2} \mathrm{H}_{6}$.

\begin{tabular}{|c|c|c|c|}
\hline $\begin{array}{l}W_{\text {exp }} \\
\mathrm{m} / \mathrm{s}\end{array}$ & $\begin{array}{l}\text { TEMP } \\
\mathrm{K}\end{array}$ & $\begin{array}{l}\text { PRES } \\
\mathrm{MPa}\end{array}$ & $\begin{array}{l}\text { PRES } \\
\text { psia }\end{array}$ \\
\hline 412.48 & 250.000 & 10.152 & 1472.4 \\
\hline 389.29 & 250.000 & 9.680 & 1404.0 \\
\hline 339.28 & 250.000 & 8.731 & 1266.3 \\
\hline 291.56 & 250.000 & 7.718 & 1119.4 \\
\hline 291.53 & 250.000 & 7.716 & 1119.1 \\
\hline 276.29 & 250.000 & 7.007 & 1016.3 \\
\hline 282.32 & 249.998 & 5.989 & 868.7 \\
\hline 276.47 & 250.000 & 6.636 & 962.4 \\
\hline 293.47 & 250.000 & 5.104 & 740.3 \\
\hline 299.59 & 250.000 & 4.664 & 676.4 \\
\hline 308.87 & 250.000 & 4.001 & 580.2 \\
\hline 318.30 & 250.000 & 3.320 & 481.6 \\
\hline 327.86 & 250.000 & 2.610 & 378.6 \\
\hline 337.22 & 250.000 & 1.895 & 274.9 \\
\hline 345.83 & 250.000 & 1.212 & 175.7 \\
\hline 353.74 & 250.000 & 0.559 & 81.1 \\
\hline 347.09 & 274.994 & 10.284 & 1491.6 \\
\hline 342.73 & 275.000 & 10.030 & 1454.7 \\
\hline 342.60 & 275.000 & 10.023 & 1453.7 \\
\hline 333.67 & 275.000 & 9.389 & 1361.7 \\
\hline 325.67 & 275.000 & 8.467 & 1228.0 \\
\hline 323.15 & 275.001 & 7.617 & 1104.7 \\
\hline 324.33 & 275.000 & 6.739 & 977.4 \\
\hline 328.94 & 275.000 & 5.732 & 831.3 \\
\hline 335.63 & 275.000 & 4.732 & 686.4 \\
\hline 343.74 & 275.000 & 3.699 & 536.5 \\
\hline 351.77 & 275.000 & 2.758 & 400.0 \\
\hline 360.95 & 275.000 & 1.715 & 248.7 \\
\hline 363.90 & 275.000 & 1.381 & 200.3 \\
\hline 367.35 & 275.000 & 0.995 & 144.4 \\
\hline 370.63 & 275.000 & 0.630 & 91.3 \\
\hline 363.87 & 300.000 & 10.662 & 1546.4 \\
\hline 361.40 & 300.000 & 10.310 & 1495.3 \\
\hline 358.06 & 300.000 & 9.707 & 1407.9 \\
\hline 355.33 & 300.000 & 8.915 & 1293.1 \\
\hline 354.22 & 300.000 & 8.294 & 1203.0 \\
\hline 354.63 & 300.000 & 7.062 & 1024.3 \\
\hline 356.67 & 300.000 & 6.198 & 899.0 \\
\hline 359.74 & 300.000 & 5.335 & 773.8 \\
\hline 364.49 & 300.005 & 4.308 & 624.8 \\
\hline 369.14 & 300.000 & 3.442 & 499.2 \\
\hline
\end{tabular}


Table 4. Speed of sound data for the mixture $0.68526 \mathrm{CH}_{4}+0.31474 \mathrm{C}_{2} \mathrm{H}_{6}$ (continued).

\begin{tabular}{|c|c|c|c|}
\hline $\begin{array}{l}W_{\exp } \\
\mathrm{m} / \mathrm{s}\end{array}$ & $\begin{array}{l}\text { TEMP } \\
\mathrm{K}\end{array}$ & $\begin{array}{l}\text { PRES } \\
\mathrm{MPa}\end{array}$ & $\begin{array}{l}\text { PRES } \\
\text { psia }\end{array}$ \\
\hline 371.54 & 299.871 & 3.016 & 437.5 \\
\hline 374.33 & 300.000 & 2.563 & 371.7 \\
\hline 375.30 & 299.866 & 2.392 & 347.0 \\
\hline 379.82 & 300.000 & 1.688 & 244.8 \\
\hline 379.55 & 299.865 & 1.719 & 249.4 \\
\hline 384.02 & 299.865 & 1.032 & 149.7 \\
\hline 384.66 & 300.000 & 0.943 & 136.8 \\
\hline 388.57 & 299.857 & 0.340 & 49.3 \\
\hline 384.49 & 325.000 & 10.635 & 1542.5 \\
\hline 384.38 & 325.000 & 10.622 & 1540.6 \\
\hline 384.06 & 325.000 & 10.530 & 1527.3 \\
\hline 383.27 & 325.000 & 10.335 & 1498.9 \\
\hline 381.43 & 325.000 & 9.693 & 1405.9 \\
\hline 379.96 & 325.000 & 8.940 & 1296.7 \\
\hline 379.29 & 325.000 & 8.289 & 1202.2 \\
\hline 379.21 & 325.000 & 7.605 & 1103.1 \\
\hline 379.74 & 325.000 & 6.940 & 1006.5 \\
\hline 380.70 & 325.000 & 6.248 & 906.1 \\
\hline 382.24 & 325.000 & 5.551 & 805.0 \\
\hline 384.17 & 325.000 & 4.829 & 700.4 \\
\hline 386.42 & 325.000 & 4.138 & 600.2 \\
\hline 388.94 & 325.000 & 3.439 & 498.8 \\
\hline 391.75 & 325.000 & 2.747 & 398.4 \\
\hline 395.11 & 325.000 & 2.009 & 291.4 \\
\hline 397.99 & 325.000 & 1.379 & 200.0 \\
\hline 398.77 & 325.000 & 1.237 & 179.5 \\
\hline 400.16 & 325.000 & 0.954 & 138.4 \\
\hline 401.49 & 325.000 & 0.687 & 99.6 \\
\hline 389.36 & 324.952 & 3.331 & 483.1 \\
\hline 392.59 & 324.961 & 2.558 & 371.0 \\
\hline 395.76 & 324.948 & 1.852 & 268.6 \\
\hline 399.66 & 324.944 & 1.032 & 149.7 \\
\hline 403.09 & 324.943 & 0.345 & 50.0 \\
\hline 404.96 & 350.000 & 10.680 & 1549.0 \\
\hline 403.23 & 350.000 & 9.992 & 1449.1 \\
\hline 401.75 & 350.000 & 9.130 & 1324.2 \\
\hline
\end{tabular}


Table 4. Speed of sound data for the mixture $0.68526 \mathrm{CH}_{4}+0.31474 \mathrm{C}_{2} \mathrm{H}_{6}$ (continued).

\begin{tabular}{llll}
\hline $\begin{array}{l}\mathbf{W}_{\text {exp }} \\
\mathbf{m} / \mathbf{s}\end{array}$ & $\begin{array}{l}\text { TEMP } \\
\mathbf{K}\end{array}$ & $\begin{array}{l}\text { PRES } \\
\text { MPa }\end{array}$ & $\begin{array}{l}\text { PRES } \\
\text { psia }\end{array}$ \\
\hline & & & \\
400.90 & 350.000 & 8.303 & 1204.3 \\
400.72 & 350.000 & 7.522 & 1091.0 \\
401.01 & 350.000 & 6.700 & 971.7 \\
401.97 & 350.000 & 5.727 & 830.6 \\
403.52 & 350.000 & 4.794 & 695.3 \\
405.96 & 350.000 & 3.695 & 535.9 \\
408.49 & 350.000 & 2.773 & 402.1 \\
408.51 & 350.001 & 2.753 & 399.3 \\
408.61 & 349.998 & 2.722 & 394.8 \\
411.56 & 350.003 & 1.799 & 260.9 \\
411.85 & 350.000 & 1.729 & 250.8 \\
418.16 & 349.994 & 1.220 & 176.9 \\
414.21 & 350.005 & 1.044 & 151.4 \\
414.60 & 350.000 & 0.948 & 137.5 \\
416.19 & 349.996 & 0.518 & 75.1 \\
416.20 & 349.995 & 0.515 & 74.7 \\
416.23 & 349.994 & 0.506 & 73.4 \\
416.82 & 350.005 & 0.347 & 50.3 \\
\hline \hline
\end{tabular}


Table 5. Speed of sound data for the mixture

$0.50217 \mathrm{CH}_{4}+0.49783 \mathrm{C}_{2} \mathrm{H}_{6}$.

\begin{tabular}{|c|c|c|c|}
\hline $\begin{array}{l}\mathbf{W}_{\exp } \\
\mathrm{m} / \mathrm{s}\end{array}$ & $\begin{array}{l}\text { TEMP } \\
\mathrm{K}\end{array}$ & $\begin{array}{l}\text { PRES } \\
\mathrm{MPa}\end{array}$ & $\begin{array}{l}\text { PRES } \\
\text { psia }\end{array}$ \\
\hline 285.33 & 250.000 & 2.890 & 419.1 \\
\hline 289.35 & 250.000 & 2.703 & 392.0 \\
\hline 297.52 & 250.000 & 2.310 & 335.0 \\
\hline 305.52 & 250.000 & 1.898 & 275.3 \\
\hline 314.39 & 250.000 & 1.404 & 203.7 \\
\hline 323.69 & 250.000 & 0.849 & 123.2 \\
\hline 329.23 & 250.000 & 0.504 & 73.2 \\
\hline 311.01 & 274.998 & 8.730 & 1266.3 \\
\hline 296.67 & 275.000 & 8.345 & 1210.3 \\
\hline 288.52 & 274.999 & 8.083 & 1172.4 \\
\hline 288.50 & 274.999 & 8.087 & 1172.9 \\
\hline 278.42 & 274.999 & 7.654 & 1110.2 \\
\hline 273.70 & 275.001 & 7.259 & 1052.8 \\
\hline 272.57 & 275.000 & 6.921 & 1003.8 \\
\hline 273.59 & 275.000 & 6.566 & 952.4 \\
\hline 276.67 & 275.001 & 6.123 & 888.1 \\
\hline 285.78 & 275.000 & 5.285 & 766.6 \\
\hline 296.59 & 275.000 & 4.438 & 643.7 \\
\hline 307.79 & 275.001 & 3.594 & 521.3 \\
\hline 317.06 & 275.001 & 2.874 & 416.9 \\
\hline 322.32 & 275.001 & 2.459 & 356.7 \\
\hline 327.30 & 274.999 & 2.063 & 299.2 \\
\hline 331.05 & 275.000 & 1.756 & 254.6 \\
\hline 336.81 & 275.000 & 1.261 & 182.9 \\
\hline 338.45 & 275.000 & 1.112 & 161.3 \\
\hline 322.42 & 300.000 & 9.809 & 1422.7 \\
\hline 319.74 & 300.000 & 9.588 & 1390.7 \\
\hline 318.06 & 300.000 & 9.427 & 1367.3 \\
\hline 314.51 & 300.000 & 9.029 & 1309.6 \\
\hline 314.54 & 300.000 & 9.034 & 1310.2 \\
\hline 310.63 & 299.997 & 8.328 & 1207.9 \\
\hline 309.66 & 300.000 & 7.634 & 1107.3 \\
\hline 311.26 & 300.000 & 6.831 & 990.8 \\
\hline 315.40 & 300.000 & 5.960 & 864.4 \\
\hline 320.81 & 300.000 & 5.142 & 745.8 \\
\hline 325.53 & 300.000 & 4.520 & 655.5 \\
\hline 333.02 & 299.999 & 3.606 & 522.9 \\
\hline 340.62 & 299.996 & 2.749 & 398.8 \\
\hline 344.04 & 300.000 & 2.368 & 343.5 \\
\hline 347.17 & 299.999 & 2.021 & 293.1 \\
\hline
\end{tabular}


Table 5. Sound of speed data for the mixture $0.50217 \mathrm{CH}_{4}+0.49783 \mathrm{C}_{2} \mathrm{H}_{6}$ (continued).

\begin{tabular}{|c|c|c|c|}
\hline $\begin{array}{l}W_{\exp } \\
\mathrm{m} / \mathrm{s}\end{array}$ & $\begin{array}{l}\text { TEMP } \\
\mathbf{K}\end{array}$ & $\begin{array}{l}\text { PRES } \\
\text { MPa }\end{array}$ & $\begin{array}{l}\text { PRES } \\
\text { psia }\end{array}$ \\
\hline 346.99 & 300.000 & 2.044 & 296.5 \\
\hline 350.40 & 300.000 & 1.663 & 241.2 \\
\hline 353.53 & 299.998 & 1.312 & 190.4 \\
\hline 356.71 & 300.000 & 0.954 & 138.3 \\
\hline 356.63 & 300.000 & 0.970 & 140.7 \\
\hline 360.00 & 300.000 & 0.585 & 84.8 \\
\hline 343.74 & 325.000 & 10.399 & 1508.2 \\
\hline 341.49 & 325.000 & 10.004 & 1450.9 \\
\hline 338.08 & 325.000 & 9.003 & 1305.8 \\
\hline 337.31 & 325.000 & 8.262 & 1198.2 \\
\hline 337.87 & 324.999 & 7.464 & 1082.6 \\
\hline 339.61 & 325.000 & 6.700 & 971.7 \\
\hline 342.26 & 325.000 & 5.938 & 861.2 \\
\hline 345.64 & 325.000 & 5.165 & 749.1 \\
\hline 349.49 & 325.000 & 4.414 & 640.1 \\
\hline 353.86 & 325.000 & 3.657 & 530.4 \\
\hline 358.65 & 325.000 & 2.882 & 418.0 \\
\hline 363.52 & 325.000 & 2.135 & 309.6 \\
\hline 368.69 & 325.000 & 1.370 & 198.6 \\
\hline 370.02 & 325.000 & 1.181 & 171.2 \\
\hline 371.05 & 325.000 & 1.032 & 149.6 \\
\hline 372.03 & 325.000 & 0.890 & 129.1 \\
\hline 373.14 & 325.000 & 0.730 & 105.9 \\
\hline 374.52 & 325.000 & 0.529 & 76.7 \\
\hline 361.18 & 350.000 & 9.009 & 1306.6 \\
\hline 360.83 & 350.000 & 8.273 & 1199.8 \\
\hline 361.24 & 350.000 & 7.569 & 1097.8 \\
\hline 362.34 & 350.000 & 6.772 & 982.2 \\
\hline 364.17 & 350.000 & 5.966 & 865.2 \\
\hline 366.74 & 350.000 & 5.104 & 740.3 \\
\hline 369.92 & 350.000 & 4.236 & 614.4 \\
\hline 373.38 & 350.000 & 3.410 & 494.6 \\
\hline 377.15 & 350.000 & 2.590 & 375.6 \\
\hline 381.74 & 350.000 & 1.660 & 240.8 \\
\hline 386.55 & 350.000 & 0.747 & 108.3 \\
\hline 383.89 & 350.000 & 1.246 & 180.8 \\
\hline 385.78 & 350.000 & 0.893 & 129.5 \\
\hline 387.33 & 350.000 & 0.601 & 87.2 \\
\hline
\end{tabular}


Table 6. Speed of sound data for the mixture $0.34524 \mathrm{CH}_{4}+0.65476 \mathrm{C}_{2} \mathrm{H}_{6}$.

\begin{tabular}{|c|c|c|c|}
\hline $\begin{array}{l}W_{\exp } \\
\mathrm{m} / \mathrm{s}\end{array}$ & $\begin{array}{l}\text { TEMP } \\
\mathbf{K}\end{array}$ & $\begin{array}{l}\text { PRES } \\
\text { MPa }\end{array}$ & $\begin{array}{l}\text { PRES } \\
\text { psia }\end{array}$ \\
\hline 273.84 & 250.000 & 2.099 & 304.4 \\
\hline 278.96 & 250.000 & 1.911 & 277.1 \\
\hline 285.53 & 250.000 & 1.655 & 240.0 \\
\hline 291.18 & 250.000 & 1.416 & 205.4 \\
\hline 295.23 & 250.000 & 1.241 & 180.0 \\
\hline 297.12 & 250.000 & 1.155 & 167.5 \\
\hline 298.11 & 250.000 & 1.107 & 160.5 \\
\hline 304.40 & 250.000 & 0.810 & 117.5 \\
\hline 310.82 & 250.000 & 0.488 & 70.8 \\
\hline 315.36 & 250.000 & 0.250 & 36.3 \\
\hline 258.68 & 275.000 & 4.212 & 610.9 \\
\hline 279.11 & 275.000 & 3.293 & 477.6 \\
\hline 280.09 & 275.000 & 3.239 & 469.8 \\
\hline 288.55 & 275.000 & 2.804 & 406.7 \\
\hline 297.10 & 275.000 & 2.339 & 339.3 \\
\hline 304.68 & 275.000 & 1.899 & 275.4 \\
\hline 313.18 & 275.000 & 1.379 & 200.0 \\
\hline 317.52 & 275.000 & 1.107 & 160.5 \\
\hline 319.49 & 275.000 & 0.973 & 141.2 \\
\hline 326.05 & 275.000 & 0.536 & 77.7 \\
\hline 272.93 & 300.010 & 6.454 & 936.1 \\
\hline 273.28 & 299.998 & 6.402 & 928.5 \\
\hline 276.06 & 300.001 & 6.041 & 876.2 \\
\hline 282.80 & 299.999 & 5.368 & 778.5 \\
\hline 290.83 & 300.000 & 4.676 & 678.3 \\
\hline 299.08 & 300.001 & 4.005 & 580.8 \\
\hline 307.09 & 300.001 & 3.363 & 487.8 \\
\hline 315.60 & 300.001 & 2.671 & 387.5 \\
\hline 319.53 & 300.001 & 2.346 & 340.3 \\
\hline 323.00 & 300.001 & 2.060 & 298.7 \\
\hline 326.21 & 300.000 & 1.795 & 260.3 \\
\hline 330.46 & 299.997 & 1.437 & 208.4 \\
\hline 331.99 & 300.000 & 1.303 & 188.9 \\
\hline 334.42 & 300.002 & 1.091 & 158.2 \\
\hline 335.37 & 299.999 & 1.011 & 146.7 \\
\hline 337.59 & 299.997 & 0.810 & 117.5 \\
\hline 338.68 & 300.000 & 0.718 & 104.1 \\
\hline 339.23 & 299.997 & 0.664 & 96.4 \\
\hline 340.55 & 299.999 & 0.549 & 79.6 \\
\hline 341.04 & 300.004 & 0.502 & 72.9 \\
\hline
\end{tabular}


Table 6. Speed of sound data for the mixture $0.34524 \mathrm{CH}_{4}+0.65476 \mathrm{C}_{2} \mathrm{H}_{6}$ (continued).

\begin{tabular}{|c|c|c|c|}
\hline $\begin{array}{l}W_{\text {exp }} \\
m / s\end{array}$ & $\begin{array}{l}\text { TEMP } \\
\mathrm{K}\end{array}$ & $\begin{array}{l}\text { PRES } \\
\mathrm{MPa}\end{array}$ & $\begin{array}{l}\text { PRES } \\
\text { psia }\end{array}$ \\
\hline 317.37 & 325.000 & 10.368 & 1503.8 \\
\hline 313.97 & 325.000 & 10.091 & 1463.6 \\
\hline 309.96 & 325.000 & 9.702 & 1407.1 \\
\hline 304.61 & 325.000 & 8.930 & 1295.2 \\
\hline 302.50 & 325.000 & 8.174 & 1185.5 \\
\hline 305.38 & 325.000 & 6.709 & 973.1 \\
\hline 309.35 & 325.000 & 5.948 & 862.6 \\
\hline 315.04 & 325.000 & 5.105 & 740.5 \\
\hline 321.07 & 325.000 & 4.322 & 626.8 \\
\hline 327.74 & 325.000 & 3.524 & 511.1 \\
\hline 334.33 & 325.000 & 2.761 & 400.4 \\
\hline 340.59 & 325.000 & 2.059 & 298.6 \\
\hline 346.97 & 325.000 & 1.342 & 194.6 \\
\hline 348.52 & 325.000 & 1.169 & 169.5 \\
\hline 350.90 & 325.000 & 0.906 & 131.4 \\
\hline 352.46 & 325.000 & 0.730 & 105.9 \\
\hline 352.69 & 325.000 & 0.701 & 101.7 \\
\hline 333.67 & 350.000 & 10.567 & 1532.7 \\
\hline 329.23 & 350.000 & 9.388 & 1361.7 \\
\hline 328.19 & 350.000 & 8.496 & 1232.3 \\
\hline 328.95 & 350.000 & 7.607 & 1103.3 \\
\hline 331.04 & 350.000 & 6.747 & 978.6 \\
\hline 333.85 & 350.000 & 5.974 & 866.5 \\
\hline 334.83 & 350.000 & 5.752 & 834.2 \\
\hline 339.31 & 350.000 & 4.835 & 701.3 \\
\hline 344.92 & 350.000 & 3.849 & 558.2 \\
\hline 350.96 & 350.000 & 2.881 & 417.9 \\
\hline 357.35 & 350.000 & 1.923 & 278.9 \\
\hline 361.27 & 350.000 & 1.349 & 195.6 \\
\hline 361.26 & 350.000 & 1.348 & 195.5 \\
\hline 365.37 & 350.000 & 0.756 & 109.7 \\
\hline 367.31 & 350.000 & 0.480 & 69.7 \\
\hline
\end{tabular}


Table 7. Speed of sound data for the mixture $0.90016 \mathrm{CH}_{4}+0.09984 \mathrm{C}_{3} \mathrm{H}_{8}$.

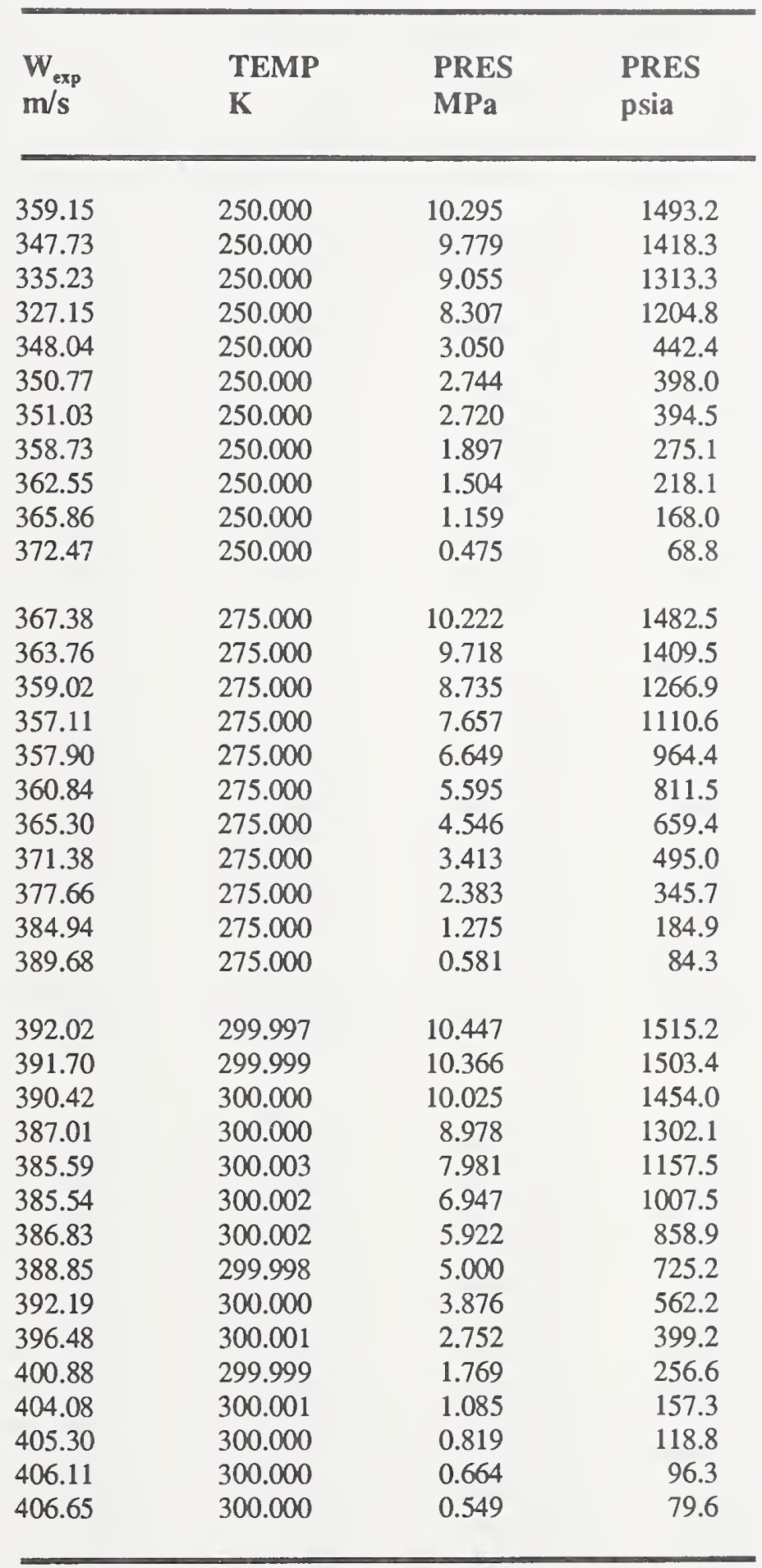


Table 7. Speed of sound data for the mixture $0.90016 \mathrm{CH}_{4}+0.09984 \mathrm{C}_{3} \mathrm{H}_{8}$ (continued).

\begin{tabular}{|c|c|c|c|}
\hline $\begin{array}{l}W_{\text {exp }} \\
\mathrm{m} / \mathrm{s}\end{array}$ & $\begin{array}{l}\text { TEMP } \\
\mathrm{K}\end{array}$ & $\begin{array}{l}\text { PRES } \\
\text { MPa }\end{array}$ & $\begin{array}{l}\text { PRES } \\
\text { psia }\end{array}$ \\
\hline 422.44 & 325.000 & 0.481 & 69.8 \\
\hline 422.26 & 325.000 & 0.524 & 76.0 \\
\hline 422.29 & 325.000 & 0.533 & 77.3 \\
\hline 421.67 & 325.000 & 0.683 & 99.1 \\
\hline 421.06 & 325.000 & 0.857 & 124.4 \\
\hline 420.39 & 325.000 & 1.035 & 150.1 \\
\hline 419.72 & 325.000 & 1.224 & 177.6 \\
\hline 418.95 & 325.000 & 1.432 & 207.7 \\
\hline 416.95 & 325.000 & 2.062 & 299.1 \\
\hline 414.48 & 325.000 & 2.951 & 428.0 \\
\hline 414.46 & 325.000 & 2.954 & 428.5 \\
\hline 414.45 & 325.000 & 2.956 & 428.7 \\
\hline 413.18 & 325.000 & 3.451 & 500.5 \\
\hline 411.50 & 325.000 & 4.122 & 597.9 \\
\hline 410.32 & 325.000 & 4.827 & 700.1 \\
\hline 409.33 & 325.000 & 5.503 & 798.1 \\
\hline 408.71 & 325.000 & 6.199 & 899.1 \\
\hline 408.35 & 325.000 & 6.889 & 999.2 \\
\hline 408.50 & 325.000 & 7.585 & 1100.1 \\
\hline 408.56 & 325.000 & 7.585 & 1100.1 \\
\hline 409.02 & 325.000 & 8.254 & 1197.1 \\
\hline 410.13 & 325.000 & 8.978 & 1302.1 \\
\hline 411.52 & 325.000 & 9.660 & 1401.1 \\
\hline 412.43 & 325.000 & 10.000 & 1450.4 \\
\hline 413.74 & 325.000 & 10.409 & 1509.6 \\
\hline 433.49 & 350.000 & 10.246 & 1486.1 \\
\hline 433.00 & 350.000 & 10.079 & 1461.8 \\
\hline 431.34 & 350.000 & 9.310 & 1350.3 \\
\hline 429.65 & 350.000 & 8.285 & 1201.7 \\
\hline 428.81 & 350.000 & 7.248 & 1051.2 \\
\hline 428.48 & 350.000 & 6.211 & 900.9 \\
\hline 428.81 & 350.000 & 5.172 & 750.1 \\
\hline 429.77 & 350.000 & 4.162 & 603.7 \\
\hline 431.24 & 350.000 & 3.104 & 450.2 \\
\hline 433.17 & 350.000 & 2.057 & 298.4 \\
\hline 434.77 & 350.000 & 1.345 & 195.1 \\
\hline 435.17 & 350.000 & 1.160 & 168.2 \\
\hline 436.00 & 350.000 & 0.821 & 119.0 \\
\hline 436.51 & 350.000 & 0.617 & 89.5 \\
\hline
\end{tabular}


Table 8. Speed of sound data for the mixture $0.95114 \mathrm{CH}_{4}+0.04886 \mathrm{~N}_{2}$.

\begin{tabular}{|c|c|c|c|}
\hline $\begin{array}{l}W_{\exp } \\
\mathrm{m} / \mathrm{s}\end{array}$ & $\begin{array}{l}\text { TEMP } \\
\mathbf{K}\end{array}$ & $\begin{array}{l}\text { PRES } \\
\mathrm{MPa}\end{array}$ & $\begin{array}{l}\text { PRES } \\
\text { psia }\end{array}$ \\
\hline 392.77 & 250.000 & 10.403 & 1508.8 \\
\hline 389.33 & 250.000 & 9.980 & 1447.5 \\
\hline 383.65 & 250.000 & 9.063 & 1314.5 \\
\hline 380.41 & 250.000 & 8.191 & 1188.0 \\
\hline 378.93 & 250.000 & 6.915 & 1003.0 \\
\hline 380.06 & 250.000 & 5.873 & 851.8 \\
\hline 382.80 & 250.000 & 4.837 & 701.5 \\
\hline 387.10 & 250.000 & 3.696 & 536.1 \\
\hline 392.26 & 250.000 & 2.596 & 376.6 \\
\hline 397.99 & 250.000 & 1.518 & 220.2 \\
\hline 402.66 & 250.000 & 0.700 & 101.5 \\
\hline 404.60 & 250.000 & 0.377 & 54.7 \\
\hline 417.79 & 275.000 & 10.460 & 1517.1 \\
\hline 415.93 & 275.000 & 10.032 & 1454.9 \\
\hline 412.22 & 275.000 & 9.009 & 1306.7 \\
\hline 409.88 & 275.000 & 7.952 & 1153.3 \\
\hline 408.93 & 275.000 & 6.949 & 1007.9 \\
\hline 409.18 & 275.000 & 5.896 & 855.2 \\
\hline 409.43 & 275.000 & 5.644 & 818.5 \\
\hline 410.41 & 275.000 & 4.843 & 702.4 \\
\hline 412.76 & 275.000 & 3.721 & 539.7 \\
\hline 415.53 & 275.000 & 2.704 & 392.2 \\
\hline 418.76 & 275.000 & 1.718 & 249.1 \\
\hline 422.29 & 275.000 & 10.772 & 112.0 \\
\hline 422.68 & 275.000 & 0.681 & 98.7 \\
\hline 440.11 & 300.000 & 9.983 & 1448.0 \\
\hline 438.29 & 300.000 & 9.399 & 1363.2 \\
\hline 436.04 & 300.000 & 8.444 & 1224.7 \\
\hline 434.63 & 300.000 & 7.583 & 1099.8 \\
\hline 433.67 & 300.000 & 6.499 & 942.6 \\
\hline 433.52 & 300.000 & 5.466 & 792.8 \\
\hline 433.81 & 300.000 & 4.799 & 696.0 \\
\hline 434.81 & 300.000 & 3.764 & 546.0 \\
\hline 436.32 & 300.000 & 2.759 & 400.2 \\
\hline 438.33 & 300.000 & 1.738 & 252.1 \\
\hline 439.87 & 300.004 & 1.092 & 158.4 \\
\hline 440.80 & 300.000 & 0.704 & 102.2 \\
\hline
\end{tabular}


Table 8. Speed of sound data for the mixture $0.95114 \mathrm{CH}_{4}+0.04886 \mathrm{~N}_{2}$ (continued).

\begin{tabular}{|c|c|c|c|}
\hline $\begin{array}{l}\mathbf{W}_{\exp } \\
\mathrm{m} / \mathrm{s}\end{array}$ & $\begin{array}{l}\text { TEMP } \\
\mathrm{K}\end{array}$ & $\begin{array}{l}\text { PRES } \\
\text { MPa }\end{array}$ & $\begin{array}{l}\text { PRES } \\
\text { psia }\end{array}$ \\
\hline 454.54 & 324.998 & 4.264 & 1618.4 \\
\hline 454.60 & 324.990 & 4.084 & 1592.3 \\
\hline 454.59 & 325.000 & 4.084 & 1592.3 \\
\hline 464.20 & 325.000 & 10.652 & 1544.9 \\
\hline 463.12 & 324.998 & 10.343 & 1500.2 \\
\hline 461.10 & 325.000 & 9.665 & 1401.8 \\
\hline 459.34 & 325.000 & 8.969 & 1300.9 \\
\hline 457.22 & 325.000 & 8.016 & 1162.7 \\
\hline 456.42 & 325.000 & 7.431 & 1077.8 \\
\hline 455.66 & 325.000 & 6.901 & 1000.8 \\
\hline 455.11 & 325.000 & 6.211 & 900.8 \\
\hline 454.70 & 325.000 & 5.524 & 801.3 \\
\hline 454.42 & 324.997 & 4.842 & 702.3 \\
\hline 454.40 & 325.000 & 4.140 & 600.4 \\
\hline 454.69 & 325.000 & 3.447 & 500.0 \\
\hline 455.24 & 325.000 & 2.753 & 399.2 \\
\hline 455.86 & 325.000 & 2.065 & 299.6 \\
\hline 456.59 & 325.000 & 1.507 & 218.5 \\
\hline 456.82 & 325.000 & 1.380 & 200.2 \\
\hline 457.02 & 325.000 & 1.237 & 179.4 \\
\hline 457.20 & 325.000 & 1.102 & 159.8 \\
\hline 457.41 & 325.000 & 0.966 & 140.1 \\
\hline 457.65 & 325.000 & 0.801 & 116.2 \\
\hline 457.88 & 325.000 & 0.670 & 97.2 \\
\hline 482.89 & 350.000 & 10.433 & 1513.2 \\
\hline 481.69 & 350.000 & 9.979 & 1447.4 \\
\hline 481.68 & 350.000 & 9.976 & 1447.0 \\
\hline 480.66 & 350.000 & 9.602 & 1392.7 \\
\hline 478.21 & 350.000 & 8.623 & 1250.6 \\
\hline 476.75 & 350.000 & 7.913 & 1147.7 \\
\hline 476.34 & 350.000 & 7.711 & 1118.4 \\
\hline 475.03 & 349.998 & 6.885 & 998.6 \\
\hline 474.07 & 350.000 & 6.139 & 890.4 \\
\hline 473.25 & 350.000 & 5.333 & 773.6 \\
\hline 472.79 & 350.000 & 4.550 & 659.9 \\
\hline 472.50 & 350.000 & 3.791 & 549.9 \\
\hline 472.44 & 350.000 & 3.028 & 439.2 \\
\hline 472.60 & 350.000 & 2.209 & 320.5 \\
\hline 473.00 & 350.000 & 1.365 & 197.9 \\
\hline
\end{tabular}


Table 8. Speed of sound data for the mixture

$0.95114 \mathrm{CH}_{4}+0.04886 \mathrm{~N}_{2}$ (continued).

\begin{tabular}{lllr}
\hline $\begin{array}{l}\text { Wexp } \\
\mathbf{m} / \mathbf{s}\end{array}$ & TEMP & $\begin{array}{l}\text { PRES } \\
\mathbf{M P a}\end{array}$ & $\begin{array}{l}\text { PRES } \\
\text { psia }\end{array}$ \\
\hline & & & \\
\hline 473.07 & 350.000 & 1.363 & 197.8 \\
473.11 & 350.000 & 1.184 & 171.8 \\
473.23 & 350.000 & 1.000 & 145.1 \\
473.40 & 350.000 & 0.781 & 113.3 \\
473.71 & 350.000 & 0.523 & 75.9 \\
& & & \\
392.61 & 249.998 & 0.281 & 40.7 \\
391.91 & 250.002 & 0.419 & 60.8 \\
391.22 & 250.000 & 0.561 & 81.4 \\
390.60 & 250.000 & 0.695 & 100.8 \\
387.71 & 250.000 & 1.320 & 191.5 \\
384.72 & 250.000 & 2.002 & 290.4 \\
381.31 & 250.001 & 2.876 & 417.1 \\
379.16 & 250.001 & 3.472 & 503.5 \\
377.29 & 250.000 & 4.091 & 593.4 \\
375.52 & 249.997 & 4.826 & 699.9 \\
374.16 & 249.999 & 5.624 & 815.7 \\
373.58 & 249.999 & 6.864 & 995.5 \\
375.41 & 250.000 & 8.108 & 1176.0 \\
378.39 & 249.998 & 8.983 & 1302.9 \\
381.34 & 249.999 & 9.591 & 1391.0 \\
383.50 & 250.001 & 9.952 & 1443.5 \\
385.71 & 250.001 & 10.281 & 1491.1 \\
& & & \\
\hline \hline
\end{tabular}


Table 9. Speed of sound data for the mixture $0.85130 \mathrm{CH}_{4}+0.14870 \mathrm{~N}_{2}$.

\begin{tabular}{|c|c|c|c|}
\hline $\begin{array}{l}\mathbf{W}_{\text {exp }} \\
\mathrm{m} / \mathrm{s}\end{array}$ & $\begin{array}{l}\text { TEMP } \\
\mathbf{K}\end{array}$ & $\begin{array}{l}\text { PRES } \\
\mathrm{MPa}\end{array}$ & $\begin{array}{l}\text { PRES } \\
\text { psia }\end{array}$ \\
\hline 411.12 & 275.000 & 0.290 & 42.1 \\
\hline 410.68 & 275.000 & 0.420 & 60.9 \\
\hline 410.26 & 275.000 & 0.551 & 79.9 \\
\hline 409.82 & 274.999 & 0.689 & 99.9 \\
\hline 409.73 & 275.000 & 0.702 & 101.8 \\
\hline 407.70 & 275.000 & 1.404 & 203.7 \\
\hline 405.90 & 275.000 & 2.104 & 305.2 \\
\hline 404.32 & 275.000 & 2.781 & 403.4 \\
\hline 403.01 & 274.999 & 3.452 & 500.7 \\
\hline 401.97 & 274.998 & 4.125 & 598.3 \\
\hline 401.29 & 275.002 & 4.800 & 696.2 \\
\hline 400.84 & 274.998 & 5.537 & 803.1 \\
\hline 400.81 & 274.998 & 6.216 & 901.6 \\
\hline 401.26 & 274.999 & 6.910 & 1002.2 \\
\hline 402.20 & 275.002 & 7.659 & 1110.8 \\
\hline 403.51 & 275.000 & 8.328 & 1207.9 \\
\hline 405.23 & 274.999 & 8.983 & 1302.8 \\
\hline 407.36 & 275.001 & 9.599 & 1392.3 \\
\hline 410.61 & 275.002 & 10.393 & 1507.4 \\
\hline 433.32 & 300.000 & 10.454 & 1516.2 \\
\hline 429.49 & 300.000 & 9.324 & 1352.4 \\
\hline 426.87 & 300.001 & 8.287 & 1202.0 \\
\hline 424.87 & 300.000 & 7.222 & 1047.5 \\
\hline 423.80 & 300.000 & 6.216 & 901.5 \\
\hline 423.32 & 300.000 & 4.943 & 716.9 \\
\hline 423.52 & 300.000 & 3.935 & 570.7 \\
\hline 424.04 & 300.000 & 3.075 & 446.0 \\
\hline 426.29 & 300.000 & 1.334 & 193.5 \\
\hline 427.59 & 300.000 & 0.628 & 91.1 \\
\hline 453.60 & 325.000 & 10.447 & 1515.2 \\
\hline 453.60 & 325.000 & 10.444 & 1514.8 \\
\hline 452.69 & 325.000 & 10.167 & 1474.6 \\
\hline 450.31 & 325.000 & 9.388 & 1361.6 \\
\hline 448.35 & 325.000 & 8.624 & 1250.8 \\
\hline 446.68 & 325.000 & 7.879 & 1142.7 \\
\hline 445.30 & 325.000 & 7.112 & 1031.6 \\
\hline 445.30 & 325.000 & 7.112 & 1031.4 \\
\hline 444.20 & 325.000 & 6.338 & 919.2 \\
\hline 442.86 & 325.000 & 4.890 & 709.2 \\
\hline
\end{tabular}


Table 9. Speed of sound data for the mixture

$0.85130 \mathrm{CH}_{4}+0.14870 \mathrm{~N}_{2}$ (continued).

\begin{tabular}{|c|c|c|c|}
\hline $\begin{array}{l}\mathrm{W}_{\text {exp }} \\
\mathrm{m} / \mathrm{s}\end{array}$ & $\begin{array}{l}\text { TEMP } \\
\mathrm{K}\end{array}$ & $\begin{array}{l}\text { PRES } \\
\mathrm{MPa}\end{array}$ & $\begin{array}{l}\text { PRES } \\
\text { psia }\end{array}$ \\
\hline 442.53 & 325.000 & 4.132 & 599.2 \\
\hline 442.43 & 325.000 & 3.367 & 488.3 \\
\hline 442.59 & 325.000 & 2.584 & 374.8 \\
\hline 443.01 & 325.000 & 1.792 & 259.9 \\
\hline 443.32 & 325.000 & 1.313 & 190.4 \\
\hline 443.35 & 325.000 & 1.313 & 190.4 \\
\hline 443.57 & 325.000 & 1.046 & 151.8 \\
\hline 443.79 & 325.000 & 0.787 & 114.2 \\
\hline 444.01 & 325.000 & 0.615 & 89.2 \\
\hline 472.33 & 350.000 & 10.465 & 1517.9 \\
\hline 472.20 & 350.000 & 10.463 & 1517.5 \\
\hline 472.20 & 350.000 & 10.429 & 1512.6 \\
\hline 471.15 & 350.000 & 10.100 & 1464.9 \\
\hline 468.48 & 350.000 & 9.166 & 1329.4 \\
\hline 466.20 & 350.000 & 8.239 & 1195.0 \\
\hline 464.34 & 350.000 & 7.370 & 1068.9 \\
\hline 462.75 & 350.000 & 6.499 & 942.6 \\
\hline 461.49 & 350.000 & 5.650 & 819.4 \\
\hline 460.52 & 350.000 & 4.824 & 699.6 \\
\hline 459.76 & 350.000 & 3.923 & 569.0 \\
\hline 459.33 & 350.000 & 3.233 & 468.9 \\
\hline 459.36 & 350.000 & 3.185 & 461.9 \\
\hline 459.11 & 350.000 & 2.406 & 349.0 \\
\hline 459.07 & 350.000 & 1.582 & 229.4 \\
\hline 459.08 & 350.000 & 1.355 & 196.5 \\
\hline 459.10 & 350.000 & 1.152 & 167.1 \\
\hline 459.16 & 350.000 & 0.999 & 144.8 \\
\hline 459.18 & 350.000 & 0.646 & 93.7 \\
\hline 459.21 & 350.000 & 0.640 & 92.9 \\
\hline 459.20 & 350.000 & 0.632 & 91.6 \\
\hline 459.33 & 350.000 & 0.542 & 78.7 \\
\hline
\end{tabular}


Table 10. Speed of sound data for the mixture $0.71373 \mathrm{CH}_{4}+0.28627 \mathrm{~N}_{2}$.

\begin{tabular}{|c|c|c|c|}
\hline $\begin{array}{l}W_{\exp } \\
\mathrm{m} / \mathrm{s}\end{array}$ & $\begin{array}{l}\text { TEMP } \\
\mathbf{K}\end{array}$ & $\begin{array}{l}\text { PRES } \\
\mathrm{MPa}\end{array}$ & $\begin{array}{l}\text { PRES } \\
\text { psia }\end{array}$ \\
\hline 377.04 & 250.000 & 10.065 & 1459.8 \\
\hline 376.80 & 250.006 & 10.039 & 1456.0 \\
\hline 373.22 & 250.014 & 9.322 & 1352.0 \\
\hline 369.05 & 250.001 & 8.255 & 1197.3 \\
\hline 366.40 & 249.993 & 6.977 & 1011.9 \\
\hline 365.70 & 249.994 & 5.692 & 825.6 \\
\hline 365.90 & 250.017 & 4.985 & 723.0 \\
\hline 366.24 & 250.007 & 4.735 & 686.7 \\
\hline 368.14 & 249.999 & 3.450 & 500.4 \\
\hline 371.21 & 250.004 & 2.139 & 310.3 \\
\hline 371.23 & 250.001 & 2.105 & 305.3 \\
\hline 373.72 & 249.998 & 1.269 & 184.1 \\
\hline 376.53 & 249.994 & 0.436 & 63.3 \\
\hline 377.07 & 250.000 & 0.313 & 45.4 \\
\hline 377.79 & 250.000 & 0.144 & 20.9 \\
\hline 377.80 & 250.000 & 0.139 & 20.2 \\
\hline 378.19 & 250.000 & 0.085 & 12.3 \\
\hline 399.50 & 275.013 & 9.922 & 1439.0 \\
\hline 396.10 & 274.998 & 8.945 & 1297.3 \\
\hline 394.43 & 275.001 & 8.301 & 1204.0 \\
\hline 392.15 & 274.996 & 7.430 & 1077.6 \\
\hline 390.53 & 274.999 & 6.258 & 907.7 \\
\hline 389.98 & 274.998 & 5.597 & 811.8 \\
\hline 389.83 & 275.044 & 5.158 & 748.2 \\
\hline 389.75 & 275.001 & 4.188 & 607.4 \\
\hline 390.15 & 275.000 & 3.450 & 500.3 \\
\hline 390.22 & 275.029 & 3.427 & 497.1 \\
\hline 390.58 & 274.997 & 3.038 & 440.6 \\
\hline 391.12 & 275.001 & 2.564 & 371.8 \\
\hline 391.27 & 274.998 & 2.446 & 354.7 \\
\hline 391.77 & 274.997 & 2.045 & 296.6 \\
\hline 392.90 & 275.000 & 1.345 & 195.1 \\
\hline 394.07 & 274.999 & 0.738 & 107.1 \\
\hline 394.91 & 275.004 & 0.347 & 50.3 \\
\hline 422.85 & 301.207 & 10.260 & 1488.1 \\
\hline 420.79 & 301.234 & 9.662 & 1401.3 \\
\hline 416.82 & 301.200 & 8.332 & 1208.5 \\
\hline 413.99 & 301.177 & 7.013 & 1017.2 \\
\hline 411.78 & 301.144 & 5.567 & 807.4 \\
\hline 410.81 & 299.998 & 5.479 & 794.7 \\
\hline
\end{tabular}


Table 10. Speed of sound data for the mixture

$0.71373 \mathrm{CH}_{4}+0.28627 \mathrm{~N}_{2}$ (continued).

\begin{tabular}{|c|c|c|c|}
\hline $\begin{array}{l}W_{\exp } \\
\mathrm{m} / \mathrm{s}\end{array}$ & $\begin{array}{l}\text { TEMP } \\
\mathbf{K}\end{array}$ & $\begin{array}{l}\text { PRES } \\
\mathrm{MPa}\end{array}$ & $\begin{array}{l}\text { PRES } \\
\text { psia }\end{array}$ \\
\hline 409.87 & 299.999 & 4.156 & 602.7 \\
\hline 409.85 & 300.098 & 3.029 & 439.3 \\
\hline 410.14 & 300.077 & 2.073 & 300.6 \\
\hline 410.57 & 300.077 & 1.398 & 202.8 \\
\hline 411.17 & 300.051 & 0.713 & 103.5 \\
\hline 411.39 & 300.005 & 0.476 & 69.0 \\
\hline 411.39 & 300.005 & 0.476 & 69.0 \\
\hline 411.44 & 299.994 & 0.354 & 51.3 \\
\hline 411.43 & 300.001 & 0.363 & 52.6 \\
\hline 411.55 & 299.998 & 0.275 & 39.9 \\
\hline 411.64 & 299.999 & 0.209 & 30.3 \\
\hline 411.82 & 299.999 & 0.146 & 21.2 \\
\hline 411.97 & 299.986 & 0.103 & 15.0 \\
\hline 440.07 & 325.000 & 10.007 & 1451.5 \\
\hline 437.90 & 325.000 & 9.286 & 1346.8 \\
\hline 435.95 & 325.000 & 8.631 & 1251.9 \\
\hline 433.68 & 325.000 & 7.730 & 1121.1 \\
\hline 431.84 & 325.000 & 6.922 & 1003.9 \\
\hline 430.63 & 325.000 & 6.207 & 900.3 \\
\hline 429.54 & 325.000 & 5.511 & 799.4 \\
\hline 429.17 & 325.000 & 5.090 & 738.2 \\
\hline 428.79 & 325.000 & 4.800 & 696.2 \\
\hline 428.49 & 325.000 & 4.661 & 676.1 \\
\hline 427.96 & 325.000 & 4.000 & 580.2 \\
\hline 427.76 & 325.000 & 3.752 & 544.2 \\
\hline 427.60 & 325.000 & 3.430 & 497.5 \\
\hline 427.34 & 325.000 & 3.047 & 442.0 \\
\hline 427.25 & 325.000 & 3.047 & 441.9 \\
\hline 427.27 & 325.000 & 2.752 & 399.1 \\
\hline 427.13 & 325.000 & 2.651 & 384.6 \\
\hline 427.02 & 325.000 & 2.404 & 348.7 \\
\hline 426.95 & 325.000 & 2.067 & 299.8 \\
\hline 427.03 & 325.000 & 1.723 & 249.9 \\
\hline 426.89 & 324.999 & 1.713 & 248.4 \\
\hline 427.02 & 325.000 & 1.378 & 199.9 \\
\hline 427.08 & 325.000 & 1.341 & 194.5 \\
\hline 427.08 & 325.000 & 1.208 & 175.3 \\
\hline 427.15 & 325.000 & 0.938 & 136.0 \\
\hline 427.20 & 325.000 & 0.733 & 106.3 \\
\hline 427.11 & 325.001 & 0.693 & 100.5 \\
\hline 427.22 & 325.000 & 0.554 & 80.3 \\
\hline 427.32 & 325.000 & 0.546 & 79.2 \\
\hline
\end{tabular}


Table 10. Speed of sound data for the mixture $0.71373 \mathrm{CH}_{4}+0.28627 \mathrm{~N}_{2}$ (continued).

\begin{tabular}{llll}
\hline $\begin{array}{l}\mathbf{W}_{\text {exp }} \\
\mathbf{m} / \mathbf{s}\end{array}$ & $\begin{array}{l}\text { TEMP } \\
\mathbf{K}\end{array}$ & $\begin{array}{l}\text { PRES } \\
\mathbf{M P a}\end{array}$ & $\begin{array}{l}\text { PRES } \\
\text { psia }\end{array}$ \\
\hline & & & \\
\hline 459.14 & 350.000 & 10.469 & 1518.3 \\
459.15 & 350.000 & 10.466 & 1518.0 \\
455.52 & 350.000 & 9.320 & 1351.7 \\
452.64 & 350.000 & 8.277 & 1200.5 \\
450.14 & 350.000 & 7.236 & 1049.5 \\
447.95 & 350.000 & 6.219 & 902.0 \\
446.19 & 350.000 & 5.170 & 749.9 \\
445.63 & 350.000 & 4.827 & 700.1 \\
444.26 & 350.000 & 3.786 & 549.1 \\
443.21 & 350.000 & 2.759 & 400.2 \\
442.51 & 350.000 & 1.712 & 248.3 \\
442.28 & 350.000 & 1.343 & 194.8 \\
442.18 & 350.000 & 0.977 & 141.7 \\
442.18 & 350.000 & 0.933 & 135.4 \\
442.21 & 350.000 & 0.932 & 135.2 \\
& & & \\
\hline
\end{tabular}


Table 11. Speed of sound data for the mixture $0.94979 \mathrm{CH}_{4}+0.05021 \mathrm{CO}_{2}$.

\begin{tabular}{|c|c|c|c|}
\hline $\begin{array}{l}W_{\exp } \\
\mathrm{m} / \mathrm{s}\end{array}$ & $\begin{array}{l}\text { TEMP } \\
\mathrm{K}\end{array}$ & $\begin{array}{l}\text { PRES } \\
\mathrm{MPa}\end{array}$ & $\begin{array}{l}\text { PRES } \\
\text { psia }\end{array}$ \\
\hline 378.77 & 249.999 & 10.517 & 1525.4 \\
\hline 372.08 & 249.998 & 9.832 & 1426.0 \\
\hline 372.05 & 249.998 & 9.826 & 1425.1 \\
\hline 366.28 & 249.998 & 8.955 & 1298.8 \\
\hline 363.41 & 249.998 & 8.186 & 1187.3 \\
\hline 362.53 & 250.002 & 7.176 & 1040.8 \\
\hline 362.61 & 250.000 & 7.106 & 1030.7 \\
\hline 363.67 & 250.001 & 6.313 & 915.6 \\
\hline 365.83 & 250.000 & 5.545 & 804.2 \\
\hline 368.98 & 250.001 & 4.734 & 686.6 \\
\hline 372.63 & 249.999 & 3.965 & 575.1 \\
\hline 376.95 & 249.999 & 3.164 & 458.9 \\
\hline 383.13 & 250.000 & 2.112 & 306.4 \\
\hline 387.48 & 249.999 & 1.413 & 204.9 \\
\hline 389.63 & 250.000 & 1.080 & 156.7 \\
\hline 391.20 & 250.000 & 0.833 & 120.9 \\
\hline 391.15 & 249.999 & 0.842 & 122.2 \\
\hline 392.04 & 250.001 & 0.703 & 102.0 \\
\hline 392.87 & 249.999 & 0.575 & 83.4 \\
\hline 401.67 & 275.000 & 10.496 & 1522.3 \\
\hline 398.56 & 275.000 & 9.804 & 1421.9 \\
\hline 395.59 & 275.000 & 8.863 & 1285.5 \\
\hline 393.95 & 275.001 & 7.837 & 1136.6 \\
\hline 393.70 & 275.001 & 6.917 & 1003.2 \\
\hline 394.88 & 275.001 & 5.692 & 825.6 \\
\hline 396.88 & 275.000 & 4.717 & 684.2 \\
\hline 400.07 & 275.000 & 3.607 & 523.2 \\
\hline 403.45 & 275.000 & 2.647 & 383.9 \\
\hline 407.25 & 275.000 & 1.685 & 244.4 \\
\hline 410.48 & 275.000 & 0.938 & 136.1 \\
\hline 412.73 & 275.000 & 0.447 & 64.9 \\
\hline 426.09 & 300.003 & 10.604 & 1537.9 \\
\hline 423.55 & 300.000 & 9.842 & 1427.4 \\
\hline 421.42 & 300.000 & 8.978 & 1302.2 \\
\hline 420.03 & 299.999 & 8.163 & 1184.0 \\
\hline 419.09 & 300.000 & 7.119 & 1032.6 \\
\hline 419.05 & 300.000 & 6.194 & 898.4 \\
\hline 419.66 & 300.000 & 5.171 & 750.0 \\
\hline 421.01 & 300.000 & 4.132 & 599.3 \\
\hline 422.99 & 300.000 & 3.089 & 448.0 \\
\hline 425.39 & 300.000 & 2.064 & 299.4 \\
\hline
\end{tabular}


Table 11. Speed of sound data for the mixture $0.94979 \mathrm{CH}_{4}+0.05021 \mathrm{CO}_{2}$ (continued).

\begin{tabular}{|c|c|c|c|}
\hline $\begin{array}{l}W_{\exp } \\
m / s\end{array}$ & $\begin{array}{l}\text { TEMP } \\
\mathrm{K}\end{array}$ & $\begin{array}{l}\text { PRES } \\
\mathrm{MPa}\end{array}$ & $\begin{array}{l}\text { PRES } \\
\text { psia }\end{array}$ \\
\hline 428.33 & 300.000 & 1.022 & 148.2 \\
\hline 429.98 & 300.000 & 0.489 & 71.0 \\
\hline 446.04 & 325.000 & 0.599 & 86.9 \\
\hline 445.99 & 325.000 & 0.599 & 86.9 \\
\hline 445.66 & 325.000 & 0.763 & 110.7 \\
\hline 445.27 & 325.001 & 0.957 & 138.8 \\
\hline 444.76 & 325.002 & 1.234 & 179.0 \\
\hline 444.54 & 325.001 & 1.356 & 196.7 \\
\hline 444.18 & 325.000 & 1.499 & 217.5 \\
\hline 443.26 & 325.000 & 2.066 & 299.6 \\
\hline 442.38 & 325.000 & 2.746 & 398.3 \\
\hline 441.56 & 325.000 & 3.446 & 499.8 \\
\hline 440.93 & 325.002 & 4.134 & 599.5 \\
\hline 440.58 & 325.000 & 4.813 & 698.1 \\
\hline 440.44 & 325.000 & 5.518 & 800.3 \\
\hline 440.32 & 325.001 & 5.518 & 800.3 \\
\hline 440.39 & 325.000 & 5.535 & 802.8 \\
\hline 440.40 & 325.000 & 5.538 & 803.2 \\
\hline 440.47 & 325.002 & 6.203 & 899.7 \\
\hline 440.81 & 325.000 & 6.892 & 999.6 \\
\hline 440.79 & 325.000 & 6.934 & 1005.7 \\
\hline 441.09 & 325.000 & 7.251 & 1051.7 \\
\hline 441.31 & 325.000 & 7.580 & 1099.4 \\
\hline 441.83 & 325.000 & 7.935 & 1150.9 \\
\hline 442.38 & 325.000 & 8.288 & 1202.1 \\
\hline 442.42 & 325.000 & 8.310 & 1205.3 \\
\hline 443.52 & 325.000 & 8.975 & 1301.7 \\
\hline 445.01 & 325.000 & 9.609 & 1393.7 \\
\hline 445.97 & 325.000 & 10.011 & 1452.0 \\
\hline 447.10 & 325.000 & 10.384 & 1506.0 \\
\hline
\end{tabular}


Table 11. Speed of sound data for the mixture $0.94979 \mathrm{CH}_{4}+0.05021 \mathrm{CO}_{2}$ (continued).

\begin{tabular}{llll}
\hline $\begin{array}{l}\mathbf{W}_{\text {exp }} \\
\mathbf{m} / \mathbf{s}\end{array}$ & $\begin{array}{l}\text { TEMP } \\
\mathbf{K}\end{array}$ & $\begin{array}{l}\text { PRES } \\
\mathbf{M P a}\end{array}$ & $\begin{array}{l}\text { PRES } \\
\text { psia }\end{array}$ \\
\hline & & & \\
\hline 461.72 & 350.000 & 8.011 & 1162.0 \\
460.32 & 350.000 & 6.956 & 1008.9 \\
459.41 & 350.000 & 5.896 & 855.2 \\
458.91 & 350.000 & 4.809 & 697.4 \\
458.88 & 350.000 & 3.783 & 548.7 \\
459.28 & 350.000 & 2.759 & 400.1 \\
460.06 & 350.000 & 1.697 & 246.1 \\
460.42 & 350.000 & 1.319 & 191.4 \\
461.10 & 350.000 & 0.688 & 99.8 \\
\hline
\end{tabular}


Table 12. Speed of sound data for the mixture $0.85026 \mathrm{CH}_{4}+0.14974 \mathrm{CO}_{2}$.

\begin{tabular}{|c|c|c|c|}
\hline $\begin{array}{l}W_{\exp } \\
m / s\end{array}$ & $\begin{array}{l}\text { TEMP } \\
\mathrm{K}\end{array}$ & $\begin{array}{l}\text { PRES } \\
\text { MPa }\end{array}$ & $\begin{array}{l}\text { PRES } \\
\text { psia }\end{array}$ \\
\hline 345.16 & 250.002 & 10.357 & 1502.1 \\
\hline 337.33 & 250.000 & 9.628 & 1396.4 \\
\hline 332.00 & 249.998 & 8.867 & 1286.0 \\
\hline 329.32 & 249.999 & 8.096 & 1174.3 \\
\hline 328.99 & 249.999 & 7.303 & 1059.2 \\
\hline 330.33 & 249.999 & 6.536 & 948.0 \\
\hline 333.12 & 249.999 & 5.704 & 827.3 \\
\hline 336.76 & 250.000 & 4.933 & 715.4 \\
\hline 341.28 & 249.999 & 4.099 & 594.6 \\
\hline 346.48 & 249.999 & 3.246 & 470.7 \\
\hline 351.17 & 249.999 & 2.523 & 366.0 \\
\hline 354.47 & 249.999 & 2.025 & 293.7 \\
\hline 356.42 & 250.001 & 1.733 & 251.3 \\
\hline 358.79 & 249.999 & 1.384 & 200.7 \\
\hline 360.63 & 249.999 & 1.114 & 161.5 \\
\hline 362.59 & 250.000 & 0.831 & 120.6 \\
\hline 362.53 & 249.999 & 0.836 & 121.2 \\
\hline 363.55 & 249.998 & 0.684 & 99.2 \\
\hline 364.33 & 250.000 & 0.564 & 81.8 \\
\hline 366.00 & 275.000 & 10.289 & 1492.4 \\
\hline 363.49 & 275.000 & 9.676 & 1403.4 \\
\hline 360.95 & 275.000 & 8.675 & 1258.2 \\
\hline 360.33 & 275.000 & 7.046 & 1021.9 \\
\hline 361.50 & 275.000 & 6.130 & 889.0 \\
\hline 363.79 & 275.000 & 5.137 & 745.0 \\
\hline 366.99 & 275.000 & 4.099 & 594.5 \\
\hline 370.76 & 275.000 & 3.094 & 448.8 \\
\hline 375.32 & 275.000 & 2.022 & 293.3 \\
\hline 379.87 & 275.000 & 1.038 & 150.5 \\
\hline 382.61 & 275.000 & 0.475 & 68.8 \\
\hline 391.10 & 300.000 & 10.772 & 1562.3 \\
\hline 389.22 & 300.000 & 10.180 & 1476.5 \\
\hline 386.88 & 300.000 & 9.197 & 1333.9 \\
\hline 385.61 & 300.000 & 8.268 & 1199.2 \\
\hline 385.11 & 300.000 & 7.255 & 1052.2 \\
\hline 385.47 & 300.000 & 6.218 & 901.9 \\
\hline 386.73 & 300.000 & 5.149 & 746.8 \\
\hline 388.48 & 300.000 & 4.114 & 596.7 \\
\hline
\end{tabular}


Table 12. Speed of sound data for the mixture $0.85026 \mathrm{CH}_{4}+0.14974 \mathrm{CO}_{2}$ (continued).

\begin{tabular}{|c|c|c|c|}
\hline $\begin{array}{l}W_{\text {exp }} \\
\mathrm{m} / \mathrm{s}\end{array}$ & $\begin{array}{l}\text { TEMP } \\
\mathbf{K}\end{array}$ & $\begin{array}{l}\text { PRES } \\
\text { MPa }\end{array}$ & $\begin{array}{l}\text { PRES } \\
\text { psia }\end{array}$ \\
\hline 390.91 & 300.000 & 3.073 & 445.7 \\
\hline 393.76 & 300.000 & 2.039 & 295.7 \\
\hline 396.96 & 300.000 & 1.029 & 149.2 \\
\hline 398.52 & 300.000 & 0.550 & 79.8 \\
\hline 411.82 & 325.000 & 10.757 & 1560.2 \\
\hline 409.63 & 325.000 & 9.853 & 1429.0 \\
\hline 407.71 & 325.000 & 8.817 & 1278.8 \\
\hline 406.55 & 325.008 & 7.788 & 1129.6 \\
\hline 406.03 & 325.000 & 6.789 & 984.6 \\
\hline 406.11 & 325.000 & 5.712 & 828.4 \\
\hline 406.76 & 325.000 & 4.677 & 678.4 \\
\hline 407.88 & 325.000 & 3.637 & 527.5 \\
\hline 409.41 & 325.000 & 2.599 & 376.9 \\
\hline 411.47 & 325.000 & 1.513 & 219.4 \\
\hline 413.24 & 325.000 & 0.696 & 101.0 \\
\hline 430.77 & 350.000 & 10.726 & 1555.6 \\
\hline 429.50 & 350.000 & 10.173 & 1475.5 \\
\hline 427.84 & 350.000 & 9.340 & 1354.6 \\
\hline 426.17 & 350.000 & 8.274 & 1200.1 \\
\hline 425.38 & 350.000 & 7.524 & 1091.2 \\
\hline 424.77 & 350.000 & 6.760 & 980.5 \\
\hline 424.39 & 350.000 & 5.799 & 841.1 \\
\hline 424.34 & 350.000 & 4.993 & 724.1 \\
\hline 424.28 & 350.000 & 4.900 & 710.7 \\
\hline 424.51 & 350.000 & 4.203 & 609.5 \\
\hline 424.52 & 350.000 & 4.201 & 609.3 \\
\hline 424.47 & 350.000 & 4.131 & 599.2 \\
\hline 424.82 & 350.000 & 3.435 & 498.2 \\
\hline 424.94 & 350.000 & 3.367 & 488.4 \\
\hline 425.43 & 350.000 & 2.725 & 395.2 \\
\hline 425.64 & 350.000 & 2.488 & 360.8 \\
\hline 426.09 & 350.000 & 2.069 & 300.1 \\
\hline 426.46 & 350.000 & 1.698 & 246.2 \\
\hline
\end{tabular}


Table 13. Speed of sound data for the mixture $0.69944 \mathrm{CH}_{4}+0.30056 \mathrm{CO}_{2}$.

\begin{tabular}{|c|c|c|c|}
\hline $\begin{array}{l}\mathrm{W}_{\exp } \\
\mathrm{m} / \mathrm{s}\end{array}$ & $\begin{array}{l}\text { TEMP } \\
\mathbf{K}\end{array}$ & $\begin{array}{l}\text { PRES } \\
\mathrm{MPa}\end{array}$ & $\begin{array}{l}\text { PRES } \\
\text { psia }\end{array}$ \\
\hline 329.92 & 249.999 & 0.627 & 90.9 \\
\hline 329.44 & 249.999 & 0.691 & 100.3 \\
\hline 328.81 & 249.999 & 0.778 & 112.8 \\
\hline 328.41 & 250.000 & 0.819 & 118.8 \\
\hline 328.10 & 249.999 & 0.867 & 125.7 \\
\hline 326.60 & 250.000 & 1.053 & 152.7 \\
\hline 321.88 & 250.000 & 1.668 & 242.0 \\
\hline 315.51 & 250.000 & 2.480 & 359.7 \\
\hline 310.62 & 249.998 & 3.086 & 447.6 \\
\hline 308.81 & 250.000 & 3.333 & 483.4 \\
\hline 305.11 & 250.000 & 3.804 & 551.7 \\
\hline 302.32 & 250.000 & 4.162 & 603.6 \\
\hline 296.09 & 250.000 & 4.972 & 721.1 \\
\hline 290.34 & 250.000 & 5.802 & 841.6 \\
\hline 285.70 & 249.999 & 6.654 & 965.0 \\
\hline 282.96 & 249.999 & 7.483 & 1085.3 \\
\hline 283.70 & 250.001 & 8.316 & 1206.1 \\
\hline 289.25 & 250.000 & 9.086 & 1317.8 \\
\hline 301.65 & 249.999 & 9.900 & 1435.9 \\
\hline 312.22 & 249.999 & 10.401 & 1508.5 \\
\hline 318.55 & 250.001 & 10.663 & 1546.6 \\
\hline 323.06 & 275.003 & 10.401 & 1508.6 \\
\hline 320.02 & 274.998 & 9.729 & 1411.1 \\
\hline 317.80 & 274.999 & 8.863 & 1285.4 \\
\hline 317.34 & 274.998 & 8.103 & 1175.2 \\
\hline 318.04 & 275.000 & 7.291 & 1057.4 \\
\hline 319.67 & 274.999 & 6.527 & 946.7 \\
\hline 322.11 & 274.999 & 5.706 & 827.5 \\
\hline 324.99 & 275.000 & 4.944 & 717.1 \\
\hline 328.62 & 275.001 & 4.100 & 594.6 \\
\hline 332.22 & 274.999 & 3.352 & 486.2 \\
\hline 336.22 & 275.001 & 2.565 & 372.0 \\
\hline 341.01 & 275.000 & 1.664 & 241.4 \\
\hline 342.51 & 275.001 & 1.377 & 199.7 \\
\hline 344.08 & 274.999 & 1.084 & 157.3 \\
\hline 345.45 & 275.000 & 0.833 & 120.8 \\
\hline 346.18 & 274.999 & 0.699 & 101.4 \\
\hline 346.92 & 275.000 & 0.560 & 81.2 \\
\hline
\end{tabular}


Table 13. Speed of sound data for the mixture $0.69944 \mathrm{CH}_{4}+0.30056 \mathrm{CO}_{2}$ (continued).

\begin{tabular}{|c|c|c|c|}
\hline $\begin{array}{l}W_{\text {exp }} \\
\mathrm{m} / \mathrm{s}\end{array}$ & $\begin{array}{l}\text { TEMP } \\
\mathbf{K}\end{array}$ & $\begin{array}{l}\text { PRES } \\
\mathrm{MPa}\end{array}$ & $\begin{array}{l}\text { PRES } \\
\text { psia }\end{array}$ \\
\hline 346.28 & 300.001 & 10.384 & 1506.0 \\
\hline 344.55 & 300.002 & 9.598 & 1392.1 \\
\hline 343.64 & 299.998 & 8.908 & 1291.9 \\
\hline 343.22 & 299.999 & 8.100 & 1174.8 \\
\hline 343.47 & 300.001 & 7.312 & 1060.5 \\
\hline 344.28 & 299.999 & 6.538 & 948.2 \\
\hline 345.70 & 299.998 & 5.685 & 824.5 \\
\hline 347.33 & 299.997 & 4.941 & 716.7 \\
\hline 349.43 & 299.999 & 4.151 & 602.0 \\
\hline 351.82 & 300.000 & 3.357 & 486.9 \\
\hline 354.80 & 299.999 & 2.464 & 357.4 \\
\hline 356.08 & 300.000 & 2.108 & 305.8 \\
\hline 357.43 & 299.999 & 1.733 & 251.3 \\
\hline 358.73 & 300.000 & 1.383 & 200.6 \\
\hline 359.71 & 299.999 & 1.127 & 163.5 \\
\hline 360.81 & 299.999 & 0.830 & 120.4 \\
\hline 361.36 & 300.000 & 0.693 & 100.5 \\
\hline 375.61 & 325.000 & 0.575 & 83.4 \\
\hline 375.12 & 325.000 & 0.748 & 108.5 \\
\hline 374.58 & 325.000 & 0.949 & 137.7 \\
\hline 374.17 & 325.000 & 1.104 & 160.1 \\
\hline 373.78 & 325.000 & 1.241 & 179.9 \\
\hline 373.42 & 325.000 & 1.361 & 197.4 \\
\hline 372.56 & 325.000 & 1.645 & 238.7 \\
\hline 370.92 & 325.000 & 2.340 & 339.4 \\
\hline 369.24 & 325.000 & 3.101 & 449.8 \\
\hline 367.69 & 325.000 & 3.865 & 560.6 \\
\hline 366.82 & 325.000 & 4.389 & 636.6 \\
\hline 366.47 & 325.000 & 4.617 & 669.7 \\
\hline 365.22 & 325.000 & 5.387 & 781.3 \\
\hline 364.63 & 325.000 & 6.183 & 896.7 \\
\hline 364.19 & 325.000 & 6.953 & 1008.4 \\
\hline 364.08 & 325.000 & 7.736 & 1122.0 \\
\hline 364.36 & 325.000 & 8.507 & 1233.8 \\
\hline 364.95 & 325.000 & 9.254 & 1342.2 \\
\hline 366.07 & 325.000 & 10.007 & 1451.4 \\
\hline 367.13 & 325.000 & 10.445 & 1514.9 \\
\hline
\end{tabular}


Table 13. Speed of sound data for the mixture $0.69944 \mathrm{CH}_{4}+0.30056 \mathrm{CO}_{2}$ (continued).

\begin{tabular}{llll}
\hline $\begin{array}{l}\text { W } \\
\text { exp }\end{array}$ & TEMP & $\begin{array}{l}\text { PRES } \\
\text { MPa }\end{array}$ & $\begin{array}{l}\text { PRES } \\
\text { psia }\end{array}$ \\
\hline & K & & \\
\hline 385.53 & 350.000 & 10.372 & 1504.3 \\
384.99 & 350.000 & 9.996 & 1449.8 \\
384.25 & 350.000 & 9.415 & 1365.6 \\
383.26 & 350.000 & 8.541 & 1238.8 \\
382.55 & 350.000 & 7.602 & 1102.6 \\
382.32 & 350.000 & 6.782 & 983.6 \\
382.44 & 350.000 & 5.865 & 850.6 \\
382.85 & 350.000 & 4.937 & 716.0 \\
383.75 & 350.000 & 3.863 & 560.2 \\
384.80 & 350.000 & 2.961 & 429.4 \\
386.10 & 350.000 & 2.063 & 299.3 \\
387.41 & 350.000 & 1.258 & 182.5 \\
388.06 & 350.000 & 0.898 & 130.3 \\
388.50 & 350.000 & 0.632 & 91.6 \\
388.53 & 350.000 & 0.631 & 91.5 \\
\hline
\end{tabular}


Table 14. Speed of sound data for mixture $0.49593 \mathrm{~N}_{2}+0.50407 \mathrm{CO}_{2}$.

\begin{tabular}{|c|c|c|c|}
\hline $\begin{array}{l}W_{\text {exp }} \\
\mathrm{m} / \mathrm{s}\end{array}$ & $\begin{array}{l}\text { TEMP } \\
\mathbf{K}\end{array}$ & $\begin{array}{l}\text { PRES } \\
\mathrm{MPa}\end{array}$ & $\begin{array}{l}\text { PRES } \\
\text { psia }\end{array}$ \\
\hline 256.91 & 250.001 & 3.956 & 573.8 \\
\hline 259.93 & 250.001 & 3.415 & 495.4 \\
\hline 262.03 & 249.998 & 3.019 & 437.8 \\
\hline 264.33 & 249.999 & 2.607 & 378.2 \\
\hline 266.54 & 249.999 & 2.202 & 319.3 \\
\hline 268.40 & 250.000 & 1.873 & 271.7 \\
\hline 270.44 & 249.999 & 1.520 & 220.4 \\
\hline 272.31 & 250.000 & 1.198 & 173.7 \\
\hline 274.29 & 249.999 & 0.836 & 121.2 \\
\hline 272.68 & 275.000 & 10.212 & 1481.1 \\
\hline 271.26 & 275.000 & 9.363 & 1357.9 \\
\hline 270.67 & 275.000 & 8.340 & 1209.6 \\
\hline 271.20 & 275.000 & 7.293 & 1057.8 \\
\hline 272.73 & 275.000 & 6.249 & 906.3 \\
\hline 274.96 & 274.999 & 5.198 & 753.9 \\
\hline 277.76 & 275.000 & 4.128 & 598.7 \\
\hline 280.91 & 275.000 & 3.091 & 448.4 \\
\hline 284.43 & 275.000 & 2.038 & 295.5 \\
\hline 294.17 & 300.000 & 10.029 & 1454.6 \\
\hline 293.69 & 300.000 & 9.685 & 1404.8 \\
\hline 292.91 & 300.000 & 9.053 & 1313.0 \\
\hline 292.31 & 300.000 & 8.270 & 1199.5 \\
\hline 292.11 & 300.000 & 7.536 & 1093.1 \\
\hline 292.38 & 300.000 & 6.554 & 950.6 \\
\hline 293.15 & 300.000 & 5.518 & 800.4 \\
\hline 294.39 & 300.000 & 4.480 & 649.8 \\
\hline 296.07 & 300.000 & 3.443 & 499.4 \\
\hline 298.13 & 300.000 & 2.391 & 346.8 \\
\hline 300.57 & 300.000 & 1.329 & 192.8 \\
\hline 311.93 & 325.000 & 10.015 & 1452.6 \\
\hline 311.49 & 325.000 & 9.675 & 1403.3 \\
\hline 311.46 & 325.000 & 9.674 & 1403.1 \\
\hline 310.04 & 325.000 & 8.201 & 1189.4 \\
\hline 310.12 & 325.000 & 8.169 & 1184.8 \\
\hline 309.66 & 325.000 & 7.396 & 1072.7 \\
\hline 309.46 & 325.000 & 6.633 & 962.1 \\
\hline 309.47 & 325.000 & 5.870 & 851.3 \\
\hline 309.64 & 325.000 & 5.115 & 741.9 \\
\hline
\end{tabular}


Table 14. Speed of sound data for mixture $0.49593 \mathrm{~N}_{2}+0.50407 \mathrm{CO}_{2}$ (continued).

\begin{tabular}{llll}
\hline $\mathbf{W}_{\text {exp }}$ & TEMP & $\begin{array}{l}\text { PRES } \\
\text { MPa }\end{array}$ & $\begin{array}{l}\text { PRES } \\
\text { psia }\end{array}$ \\
& $\mathbf{K}$ & & \\
\hline & & & \\
309.99 & 325.000 & 4.355 & 631.6 \\
310.52 & 325.000 & 3.583 & 519.7 \\
310.54 & 325.000 & 3.584 & 519.8 \\
311.18 & 325.000 & 2.837 & 411.4 \\
312.06 & 325.000 & 2.063 & 299.2 \\
312.99 & 325.000 & 1.383 & 200.6 \\
313.29 & 325.000 & 1.206 & 174.9 \\
313.56 & 325.000 & 1.030 & 149.3 \\
313.87 & 325.000 & 0.852 & 123.5 \\
314.44 & 325.000 & 0.519 & 75.3 \\
& & & \\
328.45 & 350.000 & 10.344 & 1500.3 \\
328.25 & 350.000 & 10.176 & 1475.9 \\
327.31 & 350.000 & 9.607 & 1393.4 \\
327.34 & 350.000 & 9.606 & 1393.2 \\
326.22 & 350.000 & 8.787 & 1274.5 \\
325.43 & 350.000 & 8.023 & 1163.7 \\
325.31 & 350.000 & 7.890 & 1144.4 \\
324.64 & 350.000 & 7.072 & 1025.7 \\
324.65 & 350.000 & 7.059 & 1023.8 \\
324.16 & 350.000 & 6.216 & 901.5 \\
324.19 & 350.000 & 6.213 & 901.1 \\
323.79 & 350.000 & 5.279 & 765.6 \\
323.84 & 350.000 & 4.558 & 661.2 \\
323.81 & 350.000 & 4.095 & 594.0 \\
323.94 & 350.000 & 3.364 & 488.0 \\
324.31 & 350.000 & 2.608 & 378.2 \\
324.87 & 350.000 & 1.752 & 254.1 \\
& & & \\
\hline \hline
\end{tabular}


Table 15. Speed of sound data for Gulf Coast mixture.

\begin{tabular}{|c|c|c|c|}
\hline $\begin{array}{l}W_{\exp } \\
m / s\end{array}$ & $\begin{array}{l}\text { TEMP } \\
\mathrm{K}\end{array}$ & $\begin{array}{l}\text { PRES } \\
\text { MPa }\end{array}$ & $\begin{array}{l}\text { PRES } \\
\text { psia }\end{array}$ \\
\hline 387.91 & 250.000 & 10.708 & 1553.1 \\
\hline 387.86 & 250.000 & 10.700 & 1551.9 \\
\hline 384.19 & 250.000 & 10.409 & 1509.7 \\
\hline 380.41 & 250.000 & 10.065 & 1459.8 \\
\hline 375.87 & 250.000 & 9.571 & 1388.2 \\
\hline 370.18 & 250.000 & 8.672 & 1257.8 \\
\hline 367.62 & 250.000 & 7.853 & 1139.0 \\
\hline 367.24 & 250.000 & 6.923 & 1004.1 \\
\hline 367.43 & 250.000 & 6.802 & 986.5 \\
\hline 369.27 & 250.000 & 5.912 & 857.5 \\
\hline 371.79 & 250.000 & 5.195 & 753.4 \\
\hline 375.64 & 250.000 & 4.366 & 633.3 \\
\hline 378.84 & 250.000 & 3.763 & 545.8 \\
\hline 383.80 & 250.000 & 2.920 & 423.5 \\
\hline 389.44 & 250.000 & 2.036 & 295.4 \\
\hline 394.19 & 250.000 & 1.317 & 191.0 \\
\hline 399.23 & 250.000 & 0.591 & 85.7 \\
\hline 406.91 & 275.000 & 10.325 & 1497.5 \\
\hline 404.02 & 275.000 & 9.715 & 1409.0 \\
\hline 404.03 & 275.000 & 9.714 & 1409.0 \\
\hline 401.33 & 275.000 & 8.935 & 1296.0 \\
\hline 399.69 & 275.000 & 8.165 & 1184.2 \\
\hline 399.00 & 275.000 & 7.369 & 1068.8 \\
\hline 399.19 & 275.000 & 6.521 & 945.8 \\
\hline 400.41 & 275.000 & 5.623 & 815.6 \\
\hline 402.12 & 275.000 & 4.824 & 699.6 \\
\hline 404.36 & 275.000 & 4.047 & 586.9 \\
\hline 407.26 & 275.000 & 3.213 & 466.1 \\
\hline 411.32 & 275.000 & 2.197 & 318.7 \\
\hline 415.73 & 275.000 & 1.209 & 175.3 \\
\hline 419.16 & 275.000 & 0.500 & 72.5 \\
\hline 431.53 & 300.000 & 10.309 & 1495.2 \\
\hline 429.40 & 300.000 & 9.652 & 1399.8 \\
\hline 427.66 & 300.000 & 8.972 & 1301.2 \\
\hline 426.29 & 300.000 & 8.233 & 1194.1 \\
\hline 425.53 & 300.000 & 7.567 & 1097.5 \\
\hline 425.19 & 300.025 & 6.842 & 992.4 \\
\hline 425.25 & 300.004 & 6.053 & 877.9 \\
\hline 425.94 & 300.003 & 5.150 & 746.9 \\
\hline
\end{tabular}


Table 15. Speed of sound data for Gulf Coast mixture (continued).

\begin{tabular}{|c|c|c|c|}
\hline $\begin{array}{l}W_{\text {exp }} \\
\mathrm{m} / \mathrm{s}\end{array}$ & $\begin{array}{l}\text { TEMP } \\
\mathbf{K}\end{array}$ & $\begin{array}{l}\text { PRES } \\
\mathrm{MPa}\end{array}$ & $\begin{array}{l}\text { PRES } \\
\text { psia }\end{array}$ \\
\hline 427.04 & 300.000 & 4.322 & 626.8 \\
\hline 428.72 & 300.004 & 3.433 & 497.9 \\
\hline 430.59 & 300.000 & 2.631 & 381.5 \\
\hline 432.98 & 300.000 & 1.780 & 258.2 \\
\hline 435.83 & 300.000 & 0.856 & 124.1 \\
\hline 437.14 & 300.000 & 0.468 & 67.9 \\
\hline 454.25 & 325.000 & 10.382 & 1505.8 \\
\hline 452.89 & 325.000 & 9.986 & 1448.4 \\
\hline 452.00 & 325.000 & 9.655 & 1400.4 \\
\hline 450.31 & 325.000 & 8.969 & 1300.9 \\
\hline 448.99 & 325.000 & 8.224 & 1192.8 \\
\hline 448.18 & 325.000 & 7.575 & 1098.7 \\
\hline 447.51 & 325.001 & 6.904 & 1001.3 \\
\hline 447.22 & 325.001 & 6.213 & 901.1 \\
\hline 447.08 & 325.000 & 5.535 & 802.8 \\
\hline 447.34 & 325.000 & 4.839 & 701.9 \\
\hline 447.37 & 325.000 & 4.838 & 701.7 \\
\hline 447.85 & 325.000 & 4.103 & 595.2 \\
\hline 448.48 & 325.000 & 3.453 & 500.8 \\
\hline 449.22 & 325.000 & 2.759 & 400.1 \\
\hline 450.42 & 325.000 & 2.065 & 299.6 \\
\hline 451.54 & 325.000 & 1.469 & 213.1 \\
\hline 451.79 & 325.000 & 1.360 & 197.2 \\
\hline 452.11 & 325.000 & 1.201 & 174.3 \\
\hline 453.46 & 325.000 & 0.554 & 80.4 \\
\hline 453.53 & 325.000 & 0.542 & 78.6 \\
\hline 474.04 & 350.000 & 10.395 & 1507.6 \\
\hline 473.09 & 350.000 & 10.051 & 1457.8 \\
\hline 471.82 & 350.000 & 9.559 & 1386.4 \\
\hline 471.11 & 350.000 & 9.246 & 1341.0 \\
\hline 469.54 & 350.000 & 8.481 & 1230.1 \\
\hline 468.29 & 350.000 & 7.721 & 1119.8 \\
\hline 468.07 & 350.000 & 7.544 & 1094.2 \\
\hline 467.14 & 350.000 & 6.741 & 977.7 \\
\hline 466.52 & 350.000 & 5.914 & 857.7 \\
\hline 466.13 & 350.000 & 5.104 & 740.2 \\
\hline 466.04 & 350.000 & 4.336 & 628.9 \\
\hline 466.21 & 350.000 & 3.509 & 508.9 \\
\hline
\end{tabular}


Table 15. Speed of sound data for Gulf Coast mixture (continued).

\begin{tabular}{llll}
\hline $\begin{array}{l}\text { Wexp } \\
\mathbf{m} / \mathbf{s}\end{array}$ & TEMP & $\begin{array}{l}\text { PRES } \\
\mathbf{M P a}\end{array}$ & $\begin{array}{l}\text { PRES } \\
\text { psia }\end{array}$ \\
\hline & & & \\
\hline 466.65 & 350.000 & 2.692 & 390.4 \\
467.33 & 350.000 & 1.875 & 271.9 \\
468.16 & 350.000 & 1.101 & 159.7 \\
468.36 & 350.000 & 0.959 & 139.0 \\
468.56 & 350.000 & 0.820 & 118.9 \\
468.79 & 350.000 & 0.633 & 91.8 \\
& & & \\
\hline
\end{tabular}


Table 16. Speed of sound data for Amarillo mixture.

\begin{tabular}{|c|c|c|c|}
\hline $\begin{array}{l}W_{\text {exp }} \\
\mathrm{m} / \mathrm{s}\end{array}$ & $\begin{array}{l}\text { TEMP } \\
\mathrm{K}\end{array}$ & $\begin{array}{l}\text { PRES } \\
\text { MPa }\end{array}$ & $\begin{array}{l}\text { PRES } \\
\text { psia }\end{array}$ \\
\hline 381.37 & 250.000 & 10.875 & 1577.2 \\
\hline 368.83 & 250.000 & 9.889 & 1434.2 \\
\hline 361.41 & 250.000 & 9.024 & 1308.8 \\
\hline 356.65 & 250.000 & 7.988 & 1158.5 \\
\hline 355.87 & 250.000 & 6.933 & 1005.6 \\
\hline 358.11 & 250.000 & 5.878 & 852.5 \\
\hline 362.43 & 250.000 & 4.806 & 697.1 \\
\hline 367.92 & 250.000 & 3.784 & 548.8 \\
\hline 374.33 & 250.000 & 2.750 & 398.8 \\
\hline 381.31 & 250.000 & 1.713 & 248.5 \\
\hline 388.72 & 250.000 & 0.667 & 96.8 \\
\hline 396.65 & 275.009 & 10.474 & 1519.1 \\
\hline 392.67 & 275.000 & 9.699 & 1406.7 \\
\hline 388.98 & 275.019 & 8.559 & 1241.4 \\
\hline 387.62 & 275.000 & 7.606 & 1103.2 \\
\hline 387.71 & 275.000 & 6.563 & 952.0 \\
\hline 389.24 & 275.000 & 5.517 & 800.1 \\
\hline 395.47 & 275.000 & 3.415 & 495.3 \\
\hline 399.66 & 275.000 & 2.405 & 348.9 \\
\hline 404.43 & 275.000 & 1.366 & 198.1 \\
\hline 407.95 & 274.998 & 0.650 & 94.3 \\
\hline 551.16 & 298.00 & 23.390 & 3392.4 \\
\hline 519.41 & 298.00 & 21.030 & 3050.1 \\
\hline 468.08 & 298.00 & 16.869 & 2446.6 \\
\hline 431.20 & 298.00 & 12.806 & 1857.4 \\
\hline 415.23 & 298.00 & 9.531 & 1382.4 \\
\hline 411.40 & 298.00 & 6.888 & 999.0 \\
\hline 420.09 & 300.000 & 10.433 & 1513.2 \\
\hline 417.49 & 300.000 & 9.653 & 1400.0 \\
\hline 414.82 & 300.000 & 8.524 & 1236.3 \\
\hline 413.31 & 300.000 & 7.204 & 1044.8 \\
\hline 413.36 & 300.003 & 6.191 & 897.9 \\
\hline 414.17 & 300.003 & 5.174 & 750.4 \\
\hline 415.84 & 300.001 & 4.095 & 593.9 \\
\hline 418.31 & 300.000 & 2.992 & 433.9 \\
\hline 421.06 & 300.000 & 2.000 & 290.1 \\
\hline 424.29 & 300.000 & 0.986 & 143.0 \\
\hline 425.77 & 300.000 & 0.557 & 80.8 \\
\hline
\end{tabular}


Table 16. Speed of sound data for Amarillo mixture (continued).

\begin{tabular}{|c|c|c|c|}
\hline $\begin{array}{l}W_{\text {exp }} \\
\mathrm{m} / \mathrm{s}\end{array}$ & $\begin{array}{l}\text { TEMP } \\
\mathbf{K}\end{array}$ & $\begin{array}{l}\text { PRES } \\
\mathrm{MPa}\end{array}$ & $\begin{array}{l}\text { PRES } \\
\text { psia }\end{array}$ \\
\hline 441.73 & 325.000 & 0.686 & 99.5 \\
\hline 441.29 & 325.000 & 0.893 & 129.6 \\
\hline 440.96 & 325.000 & 1.036 & 150.3 \\
\hline 440.65 & 325.000 & 1.190 & 172.6 \\
\hline 440.24 & 325.000 & 1.366 & 198.1 \\
\hline 440.00 & 325.000 & 1.464 & 212.3 \\
\hline 439.53 & 325.000 & 1.654 & 239.9 \\
\hline 438.20 & 325.000 & 2.411 & 349.7 \\
\hline 437.08 & 325.000 & 3.113 & 451.5 \\
\hline 436.15 & 325.000 & 3.799 & 551.1 \\
\hline 435.96 & 325.000 & 4.136 & $59) .8$ \\
\hline 435.80 & 325.000 & 4.149 & 601.7 \\
\hline 435.94 & 325.000 & 4.149 & 601.8 \\
\hline 435.35 & 324.999 & 4.805 & 696.8 \\
\hline 435.25 & 325.000 & 5.197 & 753.8 \\
\hline 435.16 & 325.000 & 5.201 & 754.3 \\
\hline 435.22 & 325.000 & 5.543 & 803.9 \\
\hline 435.09 & 325.000 & 6.234 & 904.1 \\
\hline 435.41 & 325.000 & 6.905 & 1001.4 \\
\hline 435.99 & 325.000 & 7.590 & 1100.8 \\
\hline 436.93 & 325.000 & 8.254 & 1197.1 \\
\hline 438.12 & 325.000 & 8.975 & 1301.6 \\
\hline 439.74 & 325.000 & 9.653 & 1400.1 \\
\hline 440.74 & 325.000 & 10.002 & 1450.7 \\
\hline 442.06 & 325.002 & 10.426 & 1512.2 \\
\hline 462.27 & 350.000 & 10.642 & 1543.5 \\
\hline 459.49 & 350.000 & 9.597 & 1391.9 \\
\hline 456.82 & 350.000 & 8.331 & 1208.4 \\
\hline 455.40 & 350.000 & 7.337 & 1064.2 \\
\hline 454.28 & 350.000 & 6.185 & 897.1 \\
\hline 453.90 & 350.000 & 5.343 & 775.0 \\
\hline 453.84 & 350.000 & 5.185 & 752.0 \\
\hline 453.85 & 350.000 & 4.116 & 597.0 \\
\hline 454.13 & 350.000 & 3.435 & 498.2 \\
\hline 454.30 & 350.000 & 3.100 & 449.6 \\
\hline 455.17 & 350.000 & 2.052 & 297.6 \\
\hline 455.16 & 350.000 & 2.052 & 297.6 \\
\hline 455.97 & 350.000 & 1.338 & 194.1 \\
\hline 456.11 & 350.000 & 1.212 & 175.9 \\
\hline
\end{tabular}


Table 16. Speed of sound data for Amarillo mixture (continued).

\begin{tabular}{llll}
\hline $\begin{array}{l}\mathbf{W}_{\text {exp }} \\
\mathbf{m} / \mathbf{s}\end{array}$ & $\begin{array}{l}\text { TEMP } \\
\mathbf{K}\end{array}$ & $\begin{array}{l}\text { PRES } \\
\mathbf{M P a}\end{array}$ & $\begin{array}{l}\text { PRES } \\
\text { psia }\end{array}$ \\
\hline & & & \\
456.16 & 350.000 & 1.190 & 172.5 \\
456.25 & 350.000 & 1.155 & 167.5 \\
456.42 & 350.000 & 1.001 & 145.1 \\
456.57 & 350.000 & 0.856 & 124.1 \\
456.58 & 350.000 & 0.856 & 124.1 \\
\hline
\end{tabular}


Table 17. Speed of sound data for the Statoil dry gas mixture.

\begin{tabular}{|c|c|c|c|}
\hline $\begin{array}{l}W_{\text {exp }} \\
\mathrm{m} / \mathrm{s}\end{array}$ & $\begin{array}{l}\text { TEMP } \\
\mathbf{K}\end{array}$ & $\begin{array}{l}\text { PRES } \\
\mathrm{MPa}\end{array}$ & $\begin{array}{l}\text { PRES } \\
\text { psia }\end{array}$ \\
\hline 365.54 & 249.999 & 10.337 & 1499.3 \\
\hline 358.57 & 249.999 & 10.020 & 1453.3 \\
\hline 352.92 & 249.999 & 9.740 & 1412.7 \\
\hline 352.75 & 250.000 & 9.737 & 1412.3 \\
\hline 340.94 & 249.999 & 9.043 & 1311.5 \\
\hline 333.62 & 250.001 & 8.337 & 1209.3 \\
\hline 330.15 & 250.000 & 7.630 & 1106.6 \\
\hline 329.89 & 250.000 & 7.016 & 1017.6 \\
\hline 331.76 & 249.999 & 6.200 & 899.2 \\
\hline 335.47 & 250.000 & 5.531 & 802.2 \\
\hline 339.81 & 250.000 & 4.846 & 702.9 \\
\hline 345.05 & 249.999 & 4.152 & 602.2 \\
\hline 350.65 & 250.000 & 3.477 & 504.3 \\
\hline 356.77 & 250.000 & 2.756 & 399.8 \\
\hline 362.76 & 250.000 & 2.086 & 302.5 \\
\hline 369.42 & 250.000 & 1.325 & 192.2 \\
\hline 370.23 & 249.999 & 1.237 & 179.3 \\
\hline 371.35 & 250.000 & 1.109 & 160.8 \\
\hline 372.47 & 250.000 & 0.976 & 141.5 \\
\hline 374.09 & 250.001 & 0.799 & 115.8 \\
\hline 375.14 & 275.000 & 10.419 & 1511.2 \\
\hline 368.69 & 275.000 & 9.413 & 1365.3 \\
\hline 364.99 & 275.000 & 8.300 & 1203.9 \\
\hline 364.49 & 275.000 & 7.260 & 1052.9 \\
\hline 365.93 & 275.000 & 6.240 & 905.1 \\
\hline 369.37 & 275.000 & 5.155 & 747.7 \\
\hline 373.94 & 275.000 & 4.112 & 596.4 \\
\hline 379.43 & 275.000 & 3.060 & 443.8 \\
\hline 385.81 & 275.000 & 1.963 & 284.7 \\
\hline 392.19 & 275.000 & 0.953 & 138.2 \\
\hline 394.95 & 275.000 & 0.520 & 75.4 \\
\hline 398.02 & 299.999 & 10.385 & 1506.3 \\
\hline 397.68 & 300.000 & 10.297 & 1493.4 \\
\hline 394.17 & 300.000 & 9.209 & 1335.6 \\
\hline 393.71 & 300.000 & 8.973 & 1301.4 \\
\hline 392.40 & 300.000 & 8.121 & 1177.9 \\
\hline 392.10 & 300.000 & 7.321 & 1061.8 \\
\hline 392.51 & 299.999 & 6.486 & 940.6 \\
\hline 393.68 & 300.000 & 5.699 & 826.6 \\
\hline
\end{tabular}


Table 17. Speed of sound data for the Statoil dry gas mixture (continued).

\begin{tabular}{|c|c|c|c|}
\hline $\begin{array}{l}W_{\exp } \\
\mathrm{m} / \mathrm{s}\end{array}$ & $\begin{array}{l}\text { TEMP } \\
\mathbf{K}\end{array}$ & $\begin{array}{l}\text { PRES } \\
\mathrm{MPa}\end{array}$ & $\begin{array}{l}\text { PRES } \\
\text { psia }\end{array}$ \\
\hline 395.51 & 300.000 & 4.841 & 702.2 \\
\hline 397.77 & 300.000 & 4.023 & 583.5 \\
\hline 400.59 & 300.000 & 3.194 & 463.2 \\
\hline 403.66 & 300.000 & 2.371 & 343.9 \\
\hline 403.65 & 300.000 & 2.398 & 347.8 \\
\hline 406.42 & 300.000 & 1.738 & 252.0 \\
\hline 406.39 & 300.000 & 1.748 & 253.5 \\
\hline 406.84 & 300.000 & 1.653 & 239.8 \\
\hline 407.43 & 300.000 & 1.502 & 217.8 \\
\hline 408.07 & 300.001 & 1.357 & 196.9 \\
\hline 408.70 & 299.999 & 1.211 & 175.6 \\
\hline 409.28 & 299.999 & 1.080 & 156.6 \\
\hline 409.86 & 300.000 & 0.955 & 138.5 \\
\hline 410.39 & 300.000 & 0.834 & 120.9 \\
\hline 411.12 & 300.000 & 0.675 & 97.9 \\
\hline 411.75 & 300.000 & 0.526 & 76.3 \\
\hline 427.57 & 325.000 & 0.466 & 67.6 \\
\hline 427.03 & 325.000 & 0.631 & 91.5 \\
\hline 427.00 & 325.000 & 0.634 & 91.9 \\
\hline 426.48 & 325.000 & 0.792 & 114.8 \\
\hline 426.34 & 325.000 & 0.832 & 120.6 \\
\hline 426.00 & 325.000 & 0.935 & 135.7 \\
\hline 425.50 & 325.000 & 1.093 & 158.5 \\
\hline 424.85 & 325.000 & 1.295 & 187.8 \\
\hline 424.32 & 325.000 & 1.441 & 209.0 \\
\hline 421.62 & 325.000 & 2.352 & 341.1 \\
\hline 419.73 & 325.000 & 3.104 & 450.3 \\
\hline 418.33 & 325.000 & 3.791 & 549.9 \\
\hline 416.84 & 325.000 & 4.542 & 658.8 \\
\hline 415.87 & 325.000 & 5.239 & 759.9 \\
\hline 415.43 & 325.000 & 5.725 & 830.3 \\
\hline 415.20 & 325.000 & 5.923 & 859.0 \\
\hline 414.77 & 325.000 & 6.625 & 960.9 \\
\hline 414.88 & 325.000 & 7.306 & 1059.6 \\
\hline 415.31 & 325.000 & 8.025 & 1163.9 \\
\hline 416.24 & 325.000 & 8.721 & 1264.9 \\
\hline 417.42 & 325.000 & 9.426 & 1367.1 \\
\hline 420.13 & 325.000 & 10.399 & 1508.3 \\
\hline 420.28 & 325.000 & 10.402 & 1508.7 \\
\hline
\end{tabular}


Table 17. Speed of sound data for the

Statoil dry gas mixture (continued).

\begin{tabular}{llll}
\hline $\begin{array}{l}\text { Wexp } \\
\mathbf{m} / \mathbf{s}\end{array}$ & $\begin{array}{l}\text { TEMP } \\
\mathbf{K}\end{array}$ & $\begin{array}{l}\text { PRES } \\
\mathbf{M P a}\end{array}$ & $\begin{array}{l}\text { PRES } \\
\text { psia }\end{array}$ \\
\hline & & & \\
\hline 440.17 & 350.000 & 10.412 & 1510.1 \\
439.19 & 350.000 & 10.028 & 1454.4 \\
437.10 & 350.000 & 8.969 & 1300.8 \\
435.62 & 350.000 & 7.902 & 1146.1 \\
434.89 & 350.000 & 6.885 & 998.6 \\
434.74 & 350.000 & 5.864 & 850.4 \\
434.79 & 350.000 & 5.665 & 821.6 \\
434.89 & 350.000 & 5.589 & 810.6 \\
435.18 & 350.000 & 4.823 & 699.5 \\
436.13 & 350.000 & 3.783 & 548.7 \\
437.56 & 350.000 & 2.753 & 399.3 \\
439.35 & 350.000 & 1.715 & 248.8 \\
440.36 & 350.000 & 1.241 & 180.0 \\
& & & \\
\hline
\end{tabular}


Table 18. Speed of sound data for the Statoil Statvordgass mixture.

\begin{tabular}{|c|c|c|c|}
\hline $\begin{array}{l}W_{\text {exp }} \\
\mathrm{m} / \mathrm{s}\end{array}$ & $\begin{array}{l}\text { TEMP } \\
\mathbf{K}\end{array}$ & $\begin{array}{l}\text { PRES } \\
\text { MPa }\end{array}$ & $\begin{array}{l}\text { PRES } \\
\text { psia }\end{array}$ \\
\hline 340.98 & 300.000 & 10.379 & 1505.3 \\
\hline 340.94 & 300.001 & 10.369 & 1503.9 \\
\hline 336.19 & 300.000 & 9.662 & 1401.4 \\
\hline 332.90 & 300.000 & 8.893 & 1289.8 \\
\hline 331.49 & 300.000 & 8.201 & 1189.4 \\
\hline 331.44 & 300.000 & 7.462 & 1082.2 \\
\hline 332.72 & 300.000 & 6.704 & 972.4 \\
\hline 335.14 & 300.000 & 5.940 & 861.5 \\
\hline 338.38 & 300.000 & 5.196 & 753.6 \\
\hline 342.80 & 300.000 & 4.369 & 633.6 \\
\hline 347.60 & 300.000 & 3.576 & 518.6 \\
\hline 352.73 & 300.000 & 2.803 & 406.6 \\
\hline 359.32 & 300.000 & 1.863 & 270.2 \\
\hline 360.09 & 325.000 & 9.895 & 1435.2 \\
\hline 358.24 & 325.000 & 9.207 & 1335.3 \\
\hline 357.89 & 325.000 & 9.047 & 1312.2 \\
\hline 356.97 & 325.000 & 8.281 & 1201.1 \\
\hline 357.10 & 325.000 & 7.291 & 1057.4 \\
\hline 358.12 & 325.000 & 6.496 & 942.2 \\
\hline 359.94 & 325.000 & 5.710 & 828.2 \\
\hline 362.66 & 325.000 & 4.837 & 701.6 \\
\hline 365.89 & 325.000 & 3.999 & 580.1 \\
\hline 369.72 & 325.000 & 3.132 & 454.2 \\
\hline 374.08 & 325.000 & 2.246 & 325.8 \\
\hline 378.67 & 325.000 & 1.380 & 200.2 \\
\hline 381.90 & 325.000 & 0.799 & 115.8 \\
\hline 384.01 & 325.000 & 0.421 & 61.1 \\
\hline 382.53 & 350.000 & 10.436 & 1513.7 \\
\hline 381.64 & 350.000 & 10.110 & 1466.4 \\
\hline 381.64 & 350.000 & 10.111 & 1466.4 \\
\hline 379.81 & 350.000 & 9.217 & 1336.9 \\
\hline 378.86 & 350.000 & 8.336 & 1209.0 \\
\hline 378.69 & 350.000 & 7.922 & 1149.0 \\
\hline 378.68 & 350.000 & 7.497 & 1087.3 \\
\hline 379.11 & 350.000 & 6.711 & 973.4 \\
\hline 379.86 & 350.000 & 6.054 & 878.1 \\
\hline 381.03 & 350.000 & 5.335 & 773.8 \\
\hline 382.84 & 350.000 & 4.535 & 657.8 \\
\hline 384.94 & 350.000 & 3.767 & 546.3 \\
\hline
\end{tabular}


Table 18. Speed of sound data for the Statoil

Statvordgass mixture (continued).

\begin{tabular}{llll}
\hline $\begin{array}{l}\mathbf{W}_{\text {exp }} \\
\mathbf{m} / \mathbf{s}\end{array}$ & $\begin{array}{l}\text { TEMP } \\
\mathbf{K}\end{array}$ & $\begin{array}{l}\text { PRES } \\
\mathbf{M P a}\end{array}$ & $\begin{array}{l}\text { PRES } \\
\text { psia }\end{array}$ \\
\hline & & & \\
\hline 387.41 & 350.000 & 2.993 & 434.0 \\
390.55 & 350.000 & 2.120 & 307.4 \\
394.04 & 350.000 & 1.232 & 178.7 \\
395.40 & 350.000 & 0.901 & 130.7 \\
396.46 & 350.000 & 0.641 & 92.9 \\
\hline
\end{tabular}




\section{Table 19}

\section{Coefficients for NGAS, Equation (3).}

$$
\begin{aligned}
& \mathrm{N} 1=0.4028026168525523 \\
& \mathrm{~N} 2=1.330664037447985 \\
& \mathrm{~N} 3=-0.421496580014783 \\
& \mathrm{~N} 4=5.103260154147587 \\
& \mathrm{~N} 5=-2.129821889124908 \\
& \mathrm{~N} 6=3.564066437091546 \\
& \mathrm{~N} 7=0.009443037461497888 \\
& \mathrm{~N} 8=0.02542066675120573 \\
& \mathrm{~N} 9=-8.750142463225309 \\
& \mathrm{~N} 10=-0.001743056843897025 \\
& \mathrm{~N} 11=-0.005437139298301518 \\
& \mathrm{~N} 12=-0.05503389605760647 \\
& \mathrm{~N} 13=0.003230999234097726 \\
& \mathrm{~N} 14=0.02841672170278382 \\
& \mathrm{~N} 15=-0.03114196639310562
\end{aligned}
$$

$$
\begin{aligned}
& \mathrm{N} 16=-1.653616778945739 \\
& \mathrm{~N} 17=-0.8397783943523482 \\
& \mathrm{~N} 18=-2.974276333189398 \\
& \mathrm{~N} 19=8.677270045092660 \\
& \mathrm{~N} 20=-2.240332292886185 \\
& \mathrm{~N} 21=-2.052230326096707 \\
& \mathrm{~N} 22=-0.01331823010826499 \\
& \mathrm{~N} 23=0.545603758634985 \\
& \mathrm{~N} 24=0.5227913163185369 \\
& \mathrm{~N} 25=0.01364977052660393 \\
& \mathrm{~N} 26=-0.1259685485968104 \\
& \mathrm{~N} 27=0.05448705971188284 \\
& \mathrm{~N} 28=-0.003685997072874476 \\
& \mathrm{~N} 29=-0.02840000533935336 \\
& \mathrm{~N} 30=0.02476372085670182
\end{aligned}
$$

\section{Component}

$1=$ nitrogen

2 = carbon dioxide

3 = methane

4 = ethane

$5=$ propane

$6=$ normal butane

$7=$ isobutane

$8=$ normal pentane

9 = isopentane

$10=$ normal hexane

$11=$ normal neptane

$12=$ octane

$13=$ normal nonane

$14=$ normal decane

\section{Crit Temp}

$\varepsilon_{\mathrm{i}}$

126.26
304.212
190.555
305.334
369.85
425.16
407.85
469.7
460.4
507.3
538.4
567.5
594.54
615.5

\section{Crit Dens \\ $\sigma^{3}$}

0.4168

0.45523

0.098629

0.14545

0.5848

0.63422

0.63748

0.63

0.63

0.68

0.7

0.7

0.77

0.8
Mol Wt

28.0134

44.01

16.043

30.07

44.097

58.123

58.123

72.15

72.15

86.177

100.204

114.231

128.285

142.285 
Values of Gk:

Component

$\begin{array}{ll}1 & -0.1379680254 \\ 2 & 1.343746237 \\ 3 & 1.0 \\ 4 & 0.9954648789 \\ 5 & 1.444150683 \\ 6 & 2.262240243 \\ 7 & 1.929806053 \\ 8 & 2.14591850 \\ 9 & 1.889485197 \\ 10 & 2.042454505 \\ 11 & 2.301477499 \\ 12 & 2.361724346 \\ 13 & 2.50780996 \\ 14 & 2.611495198\end{array}$

Component

$\begin{array}{ll}1 & -5.214016927 \\ 2 & -8.398770437 \\ 3 & 1.0\end{array}$

$4 \quad 1.492840378$

$5 \quad-3.374892231$

$6 \quad-17.77894353$

$7 \quad 0.8505330491$

$8 \quad-3.227381796$

$9 \quad-0.4229016591$

$10 \quad-4.798074499$

$11 \quad-0.3922091741$

$12 \quad 0.8087676095$

$13 \quad 0.7061197568$

$14 \quad 0.1322694817$
G4

$-6.489193563$

$-0.4374445576$

1.0

0.9984104253

2.44323019

2.543607824

1.750437195

3.775088823

1.324890237

2.535866056

1.660952157

1.842959663

1.579803181

2.471380059

\section{G7}

$-55.25891745$

$-21.65642766$

1.0

5.777994243

$-8.420869082$

45.67847097

1.168709277

29.53337376

$-15.25498097$

$-11.05517674$

$-0.3846575238$

14.22796425

7.888704149

$-1.002974962$
G5

$-11.82111721$

$-6.275691346$

1.0

1.11243239

2.815679962

$-8.337742816$

0.3686574747

$-3.658214800$

0.1474343421

3.265853765

$-0.8595200515$

$-0.6012849579$

$-0.8381601652$

$-1.157843638$

G8

$-24.10351873$

$-0.7492990156$

1.0

0.9402037520

0.7041873252

0.8369555875

$-4.044894166$

$-18.66585785$

1.095166830

6.640963739

$-0.6775239571$

$-6.860930093$

$-3.737466433$

$-2.279161978$

\section{Component}

$\begin{array}{lrl}1 & 19.956846 & 43 \\ 2 & 0.2601856076 \\ 3 & 1.0 & \\ 4 & -0.299 & 5886026 \\ 5 & 0.887 & 3446878 \\ 6 & -12.32090154 \\ 7 & 6.015 & 133981 \\ 8 & 19.73846926 \\ 9 & 1.181912388 \\ 10 & -0.6637357127 \\ 11 & 1.371781956 \\ 12 & 4.627715335 \\ 13 & 2.959502580 \\ 14 & -4.102453851\end{array}$

G10

$-1602.900815$

52.022136397

1.0

$-82.22159566$

$-0.2457234145$

1829.107816

$-868.6477399$

$-2616.867323$

$-111.5835115$

$-66.81906952$

$-128.6682986$

$-942.0947444$

$-517.9166255$

$-187.9137342$
G11

957.6112759

12.68101596

1.0

$-4.544856988$

5.463328055

$-174.3629879$

655.0233236

2767.027867

118.9025629

103.6692040

120.7078503

429.3494259

268.3113027

63.29269208 
Values of interaction parameters, $u_{i j}$ :

All $\mathrm{u}_{\mathrm{ij}}=\mathrm{u}_{\mathrm{ji}}$, and all $\mathrm{u}_{\mathrm{ij}}$ not specified are 1.0.

$$
\begin{aligned}
& \mathrm{u}_{1,2}=0.5 \\
& u_{2,3}=0.8526452078 \\
& u_{1,3}=0.559847933 \\
& u_{1,4}=0.805299176 \\
& \left(\mathrm{u}_{3, \mathrm{j}}, \mathrm{j}=4 \text { to } 14\right) \\
& 0.775434442977 \\
& \mathrm{u}_{2,4}=0.9914 \\
& \mathrm{u}_{2,5}=0.9957 \\
& 0.59709366850 \\
& 1.182954569 \\
& 0.6475447996 \\
& 0.6467821411 \\
& u_{4,6}=-0.354556
\end{aligned}
$$

Values of interaction parameters, $v_{i j}$ :

All $\mathrm{v}_{\mathrm{ij}}=\mathrm{v}_{\mathrm{ji}}$, and all $\mathrm{v}_{\mathrm{ij}}$ not specified are 1.0.

$$
\begin{aligned}
& \mathrm{v}_{1,2}=0.5 \\
& v_{2,3}=0.7564367672 \\
& \left(v_{3, j}, j=4 \text { to } 14\right) \\
& 1.0205613777 \\
& v_{1,3}=0.878785412 \quad v_{1,4}=1.08642559 \\
& 0.7698806791 \\
& 0.8528924512 \\
& 0.8954629540 \\
& v_{4,5}=0.816674999
\end{aligned}
$$

Values of interaction parameters, $w_{i j}$ :

All $w_{i j}=w_{j i}$, and all $w_{i j}$ not specified are 1.0.

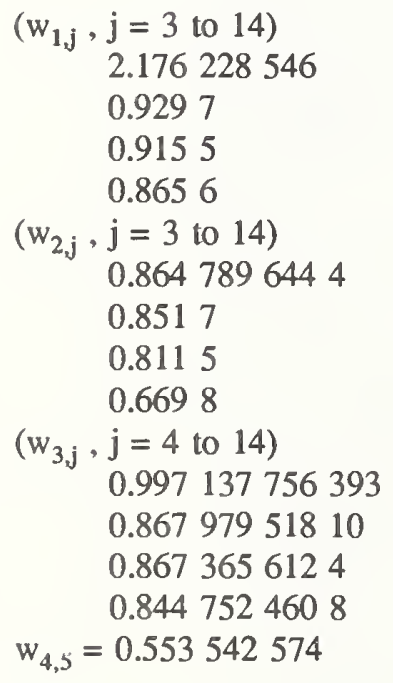

$\begin{array}{ll}0.7806439167 & 0.9416 \\ 0.9274 & 0.9161 \\ 0.8981 & 0.8806 \\ 0.8499 & 0.8335 \\ & \\ 0.9199 & 0.8944 \\ 0.8454 & 0.8131 \\ 0.762 & 0.7124 \\ 0.6252 & 0.5786 \\ & \\ 0.9219576266 & 0.9168223186 \\ 0.9037974903 & 0.8545821957 \\ 0.8474980509 & 0.8436806486 \\ 0.8349259037 & \end{array}$


Figures

00000 AGA 8

$* * * * *$ NGAS

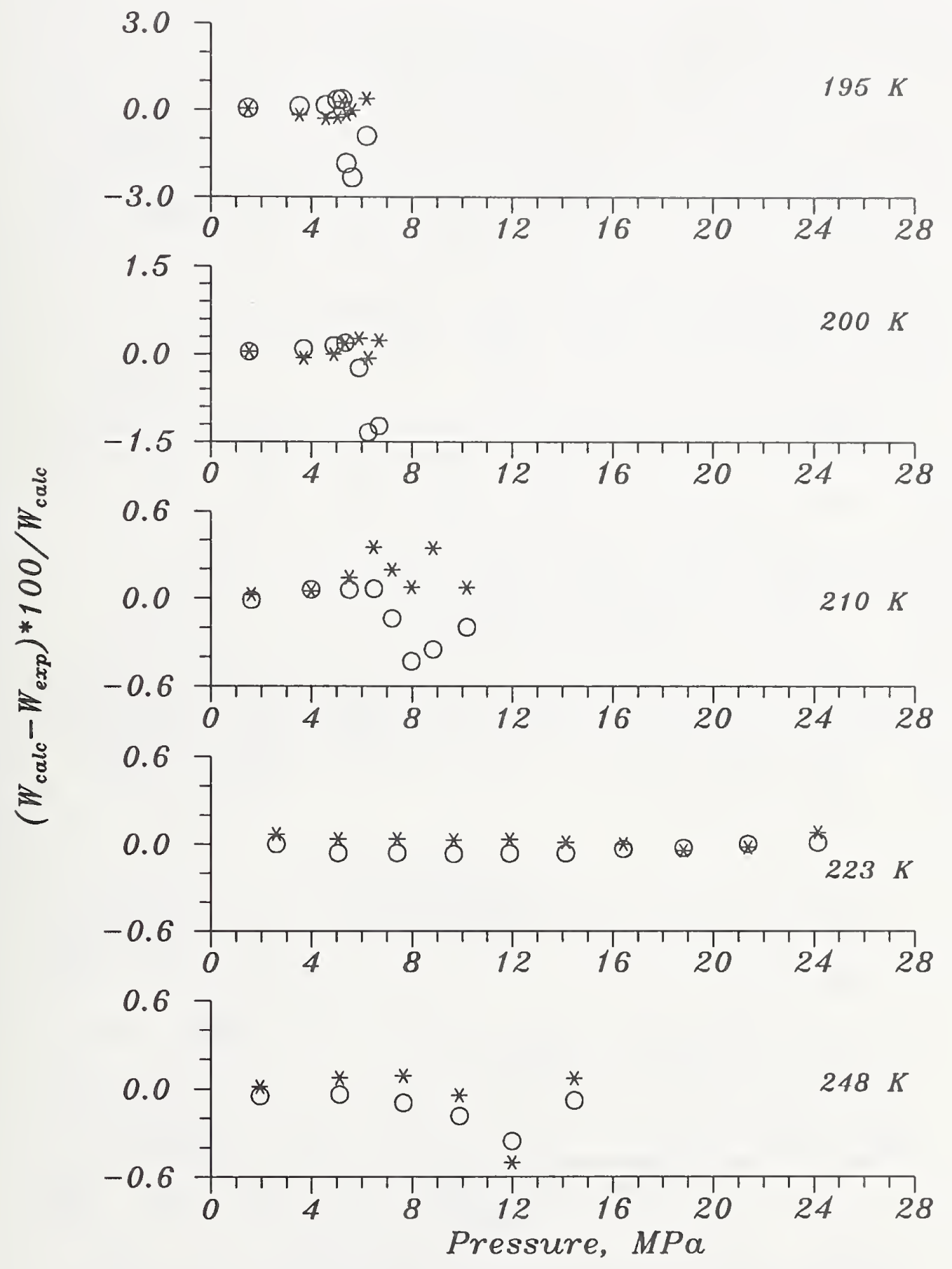

Figure 1a. Deviations of speed of sound computed by $A G A B$ and NGAS from the experimental values for the pure methare data of Sivaraman and Gammon [8]. 


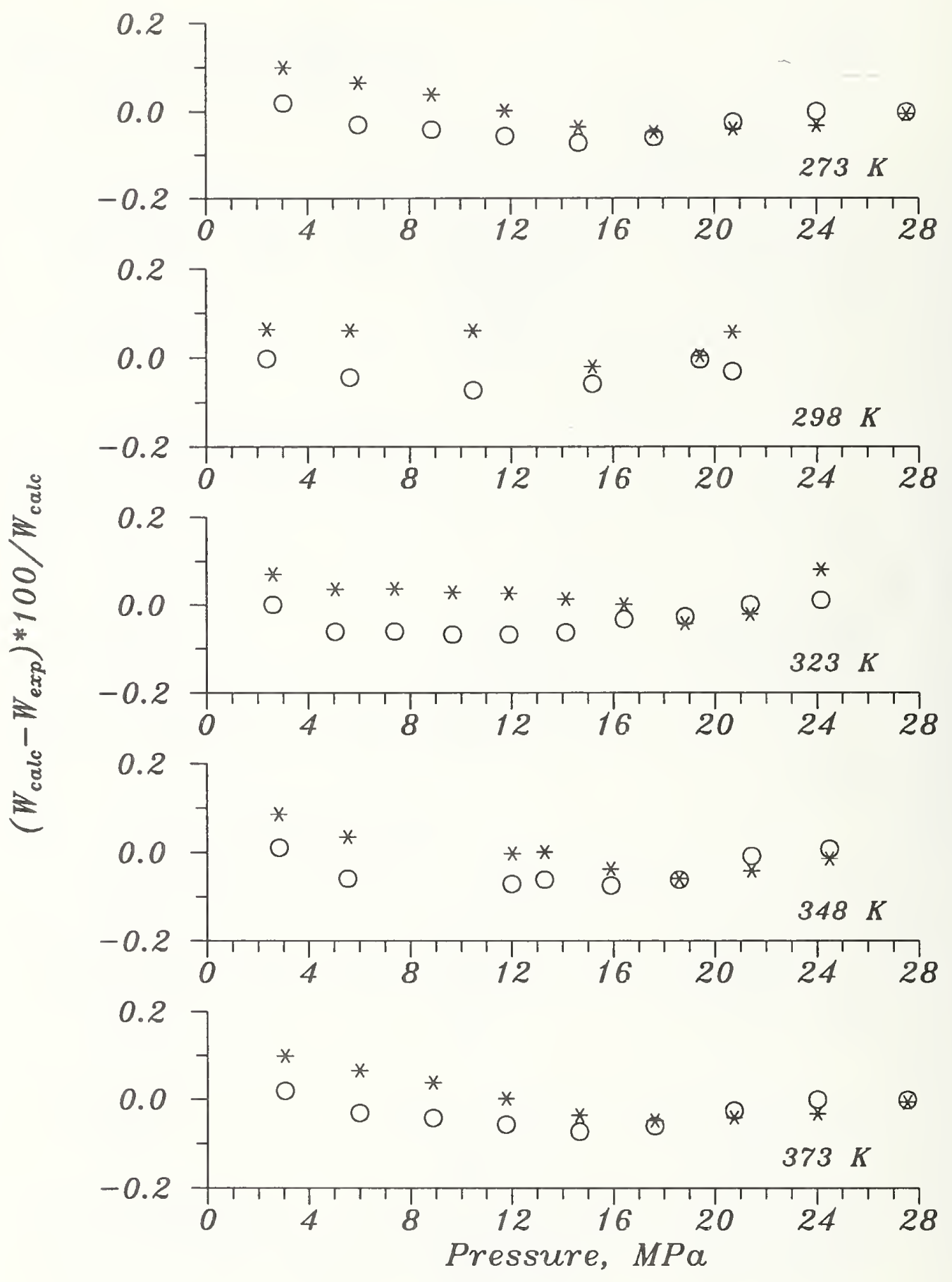

Figure 1b. Deviations of speed of sound computed by $A G A 8$ and NGAS from the experimental values for the pure methane data of Sivaraman and Gammon [8]. 
OOOOO AGA 8

$* * * * *$ NGAS

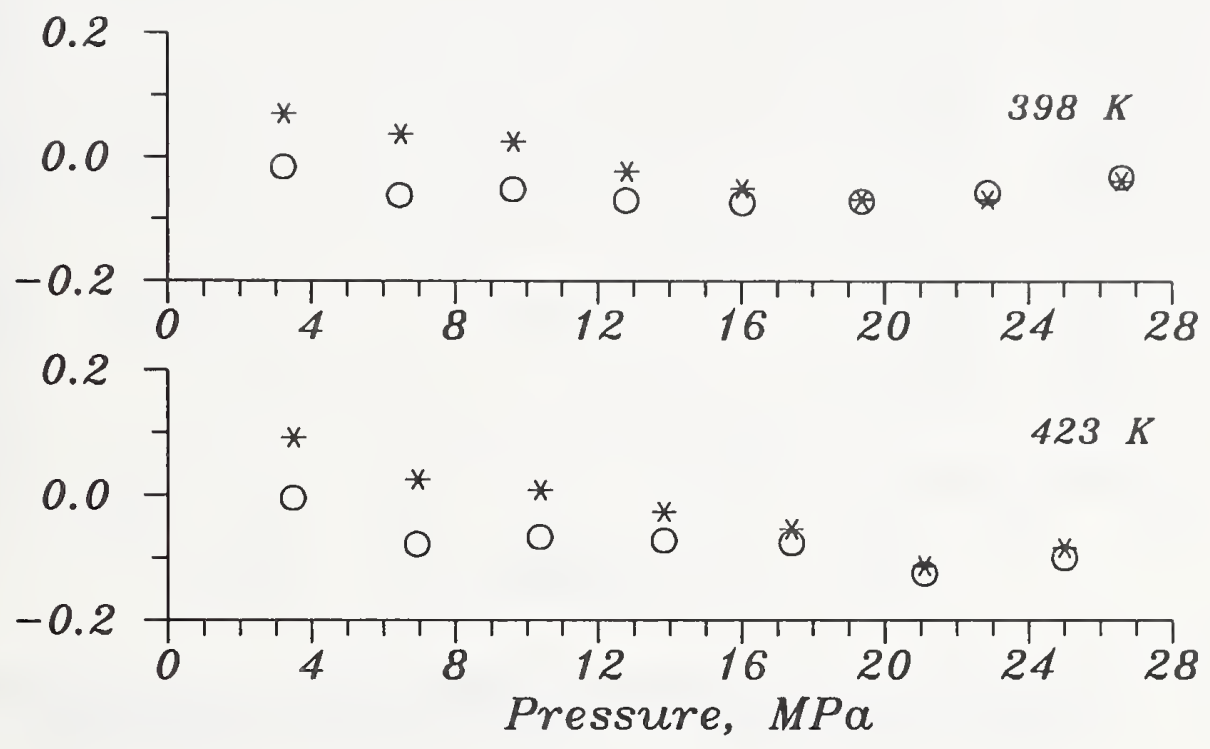

Figure 1c. Deviations of speed of sound computed by $A G A 8$ and NGAS from the experimental values for the pure methane data of Sivaraman and Gammon [8].

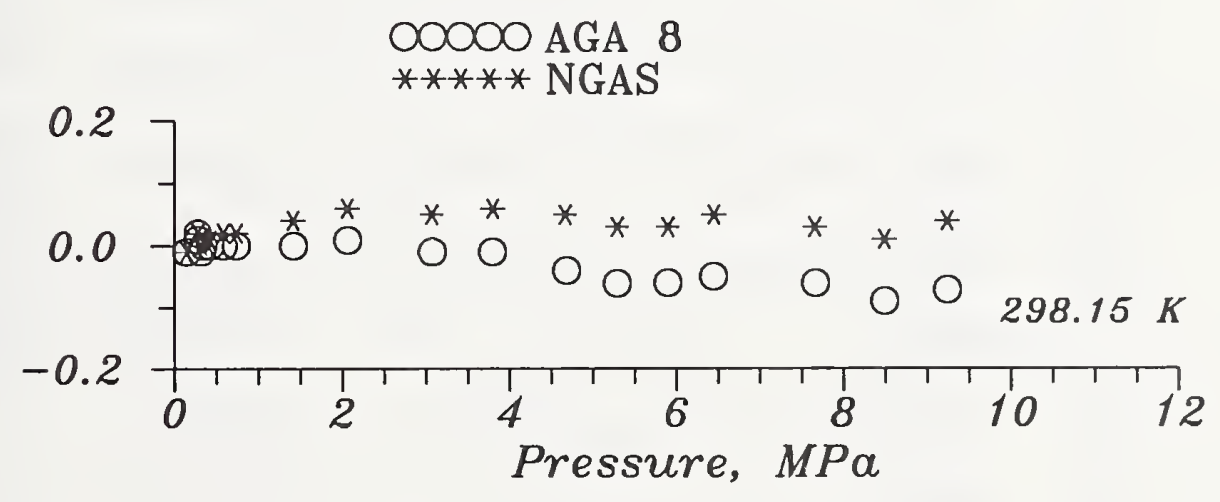

Figure 2. Deviations of speed of sound computed by $A G A 8$ and NGAS from the NIST experimental values for pure methane. 


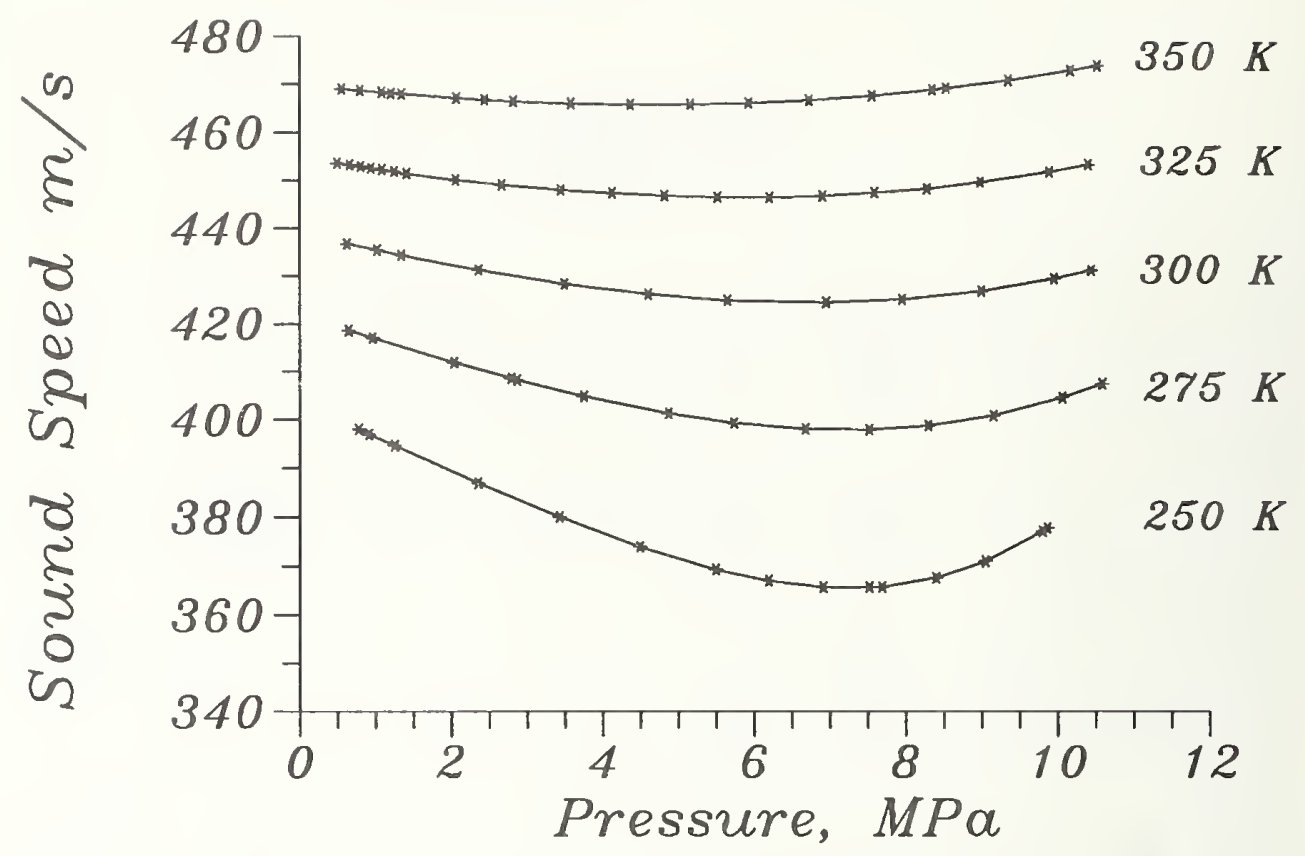

Figure 3. Experimental speed of sound for the binary mixture methane 0.95 - ethane 0.05 .

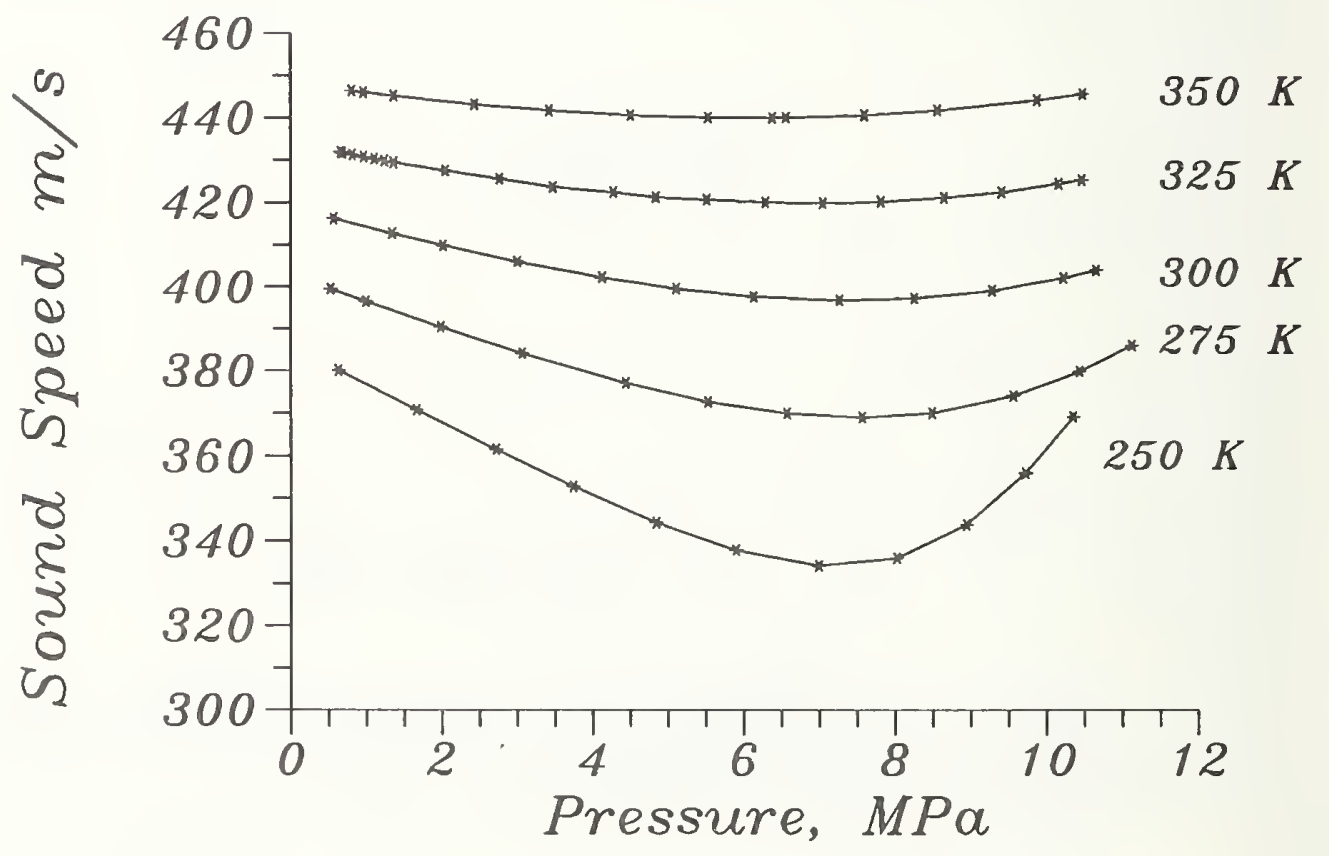

Figure 4. Experimental speed of sound for the binary mixture methane 0.85 - ethane 0.15 . 


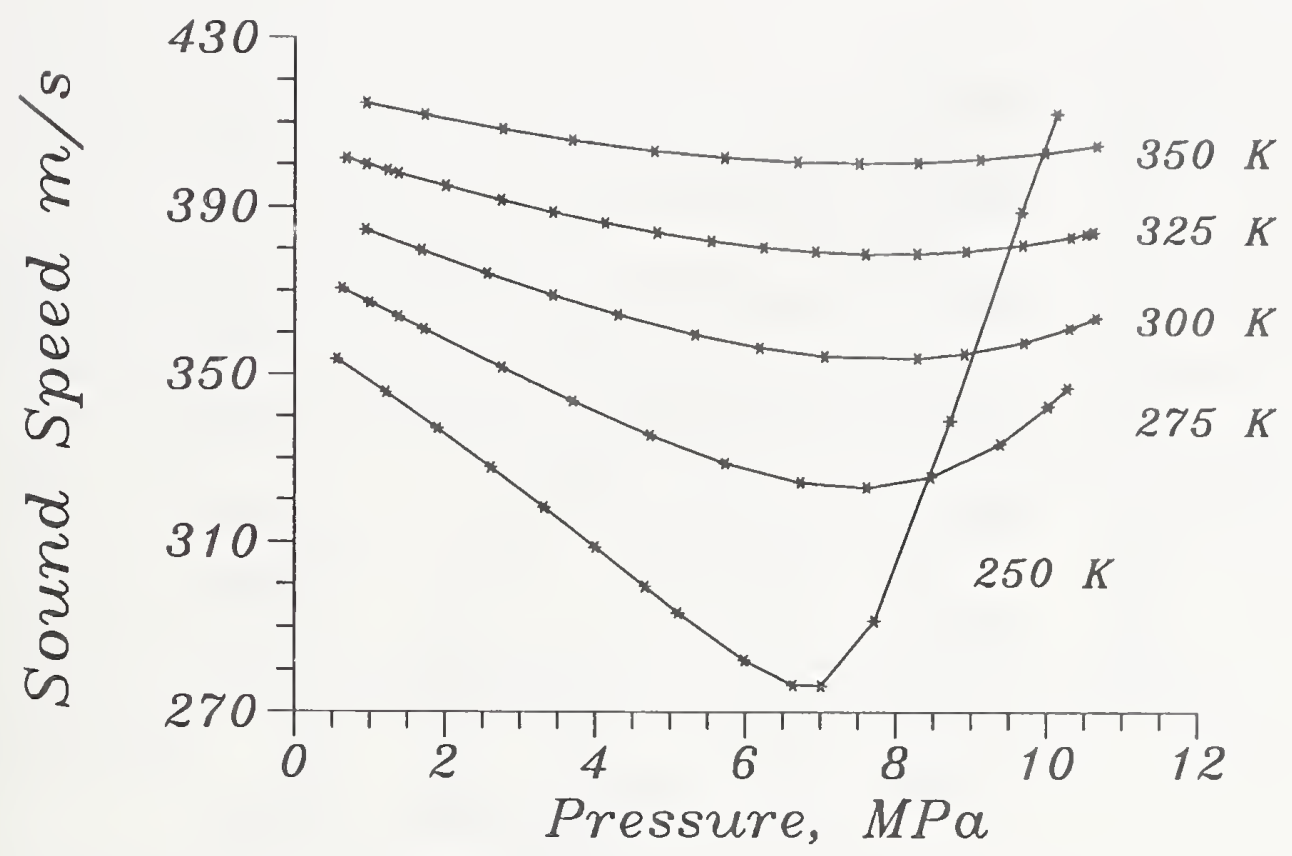

Figure 5. Experimental speed of sound for the binary mixture methane 0.69 - ethane 0.31 .

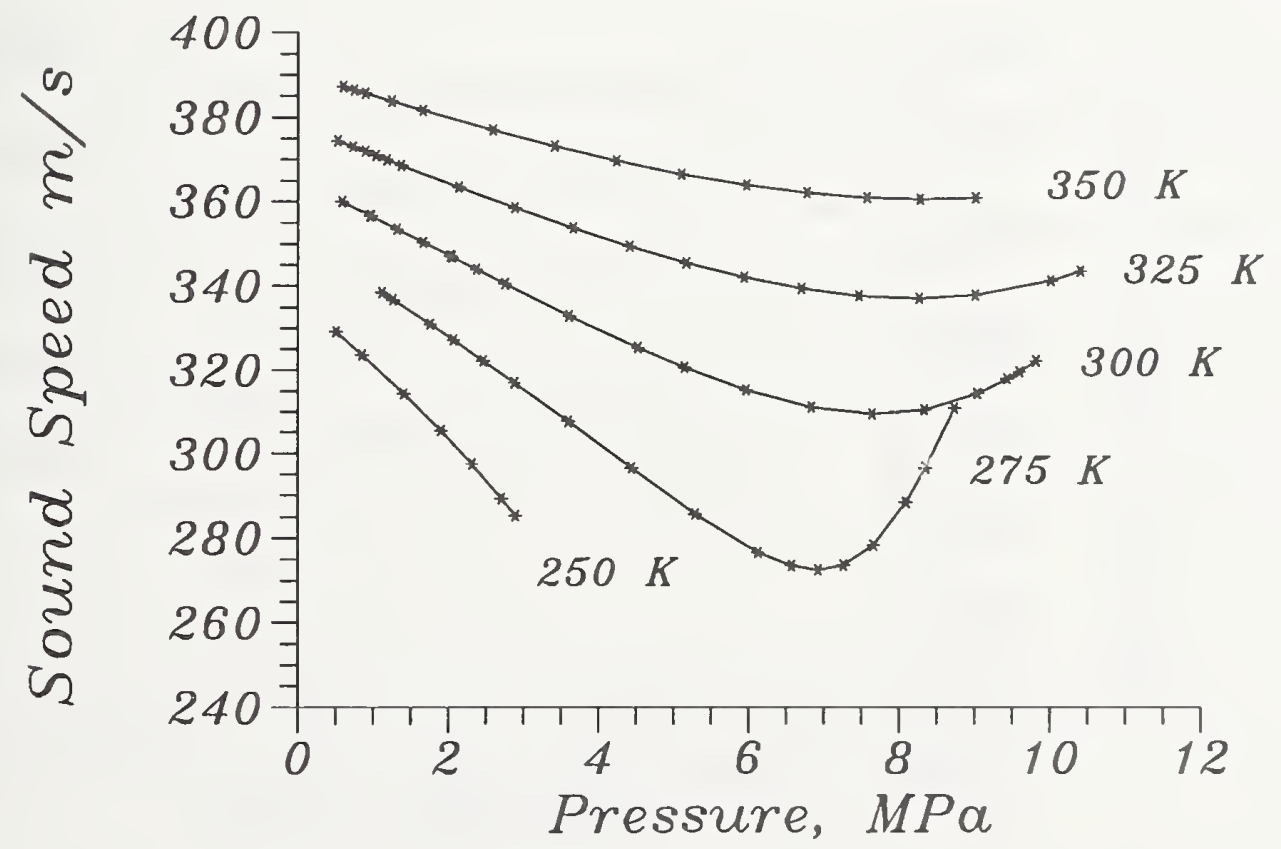

Figure 6. Experimental speed of sound for the binary mixture methane 0.50 - ethane 0.50 . 


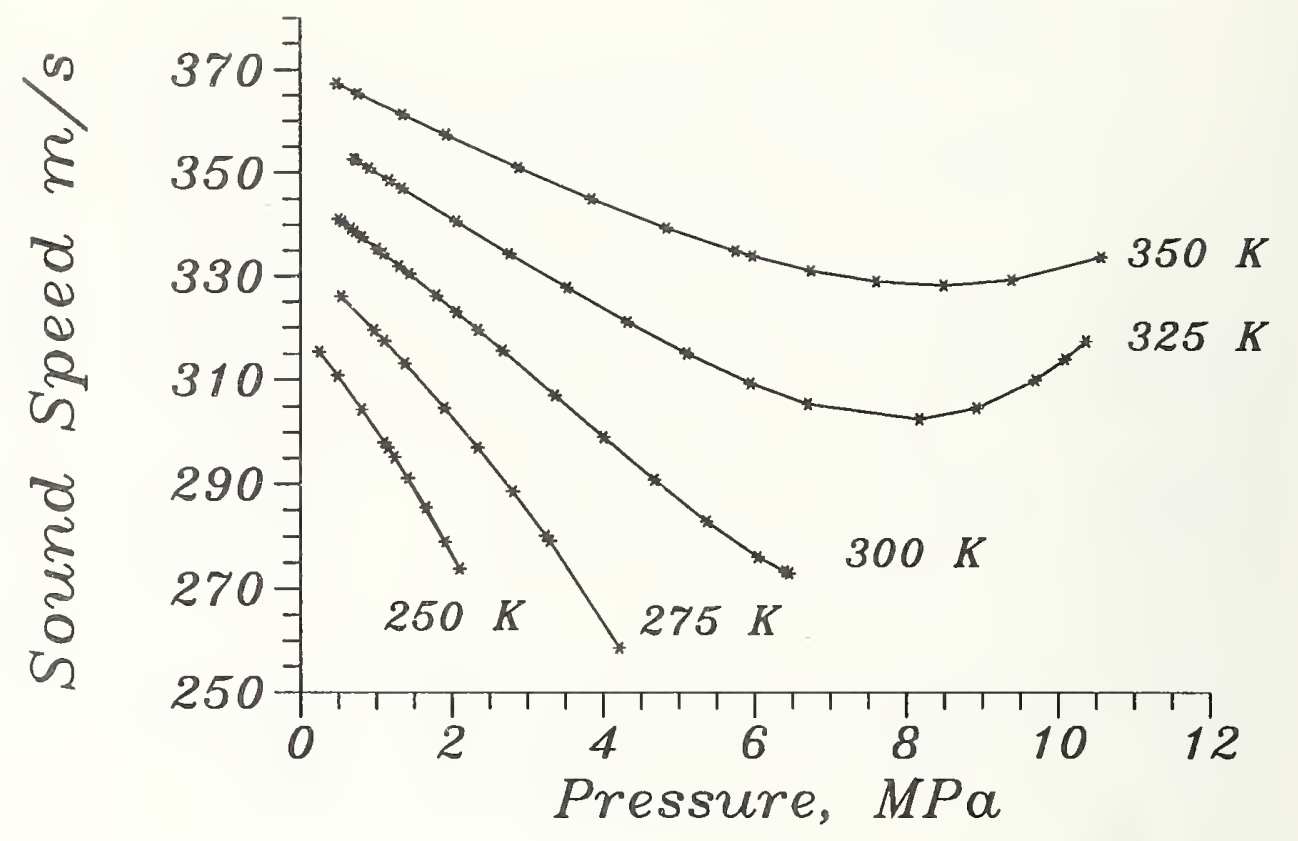

Figure 7. Experimental speed of sound for the binary mixture methane 0.35 - ethane 0.65 .

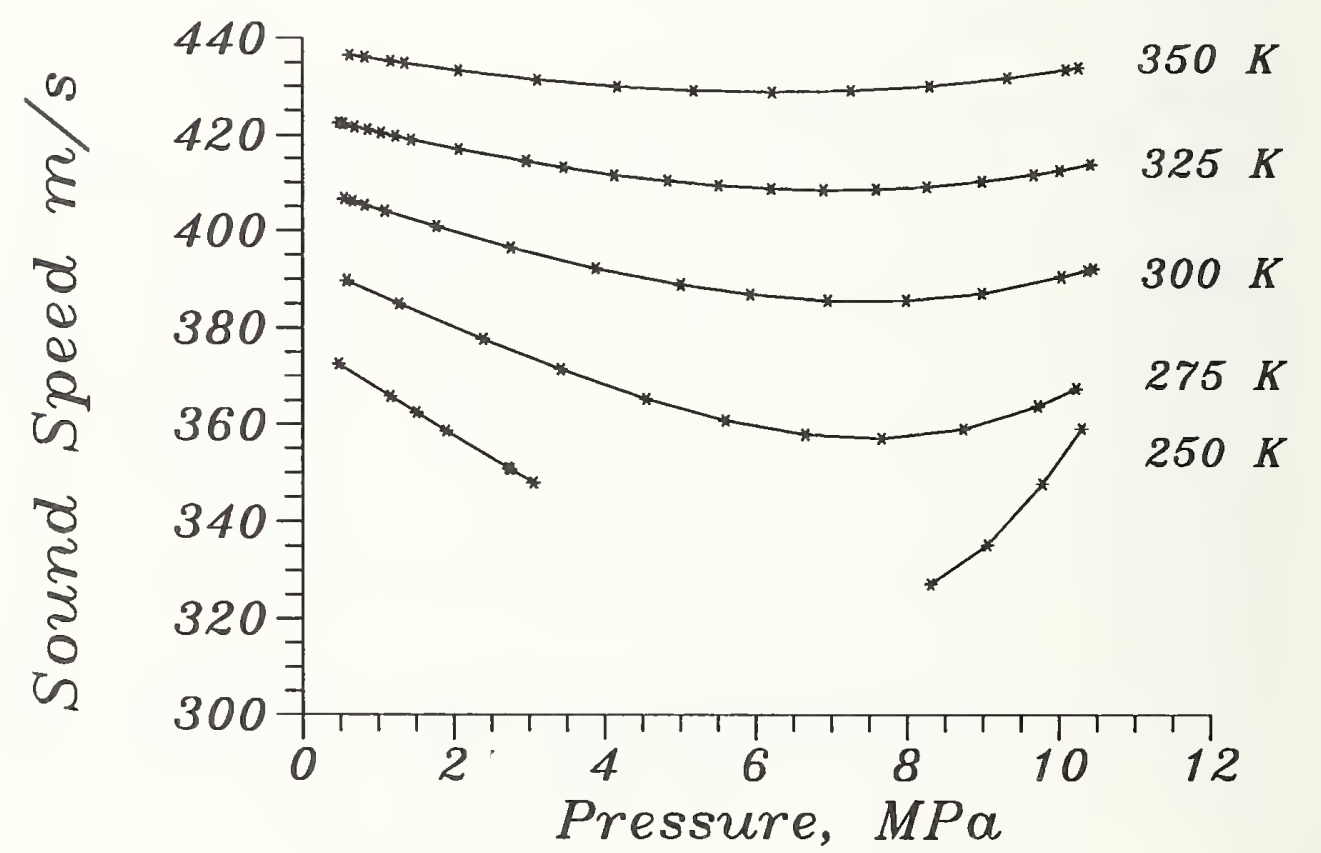

Figure 8. Experimental speed of sound for the binary mixture methane 0.90 - propane 0.10 . 


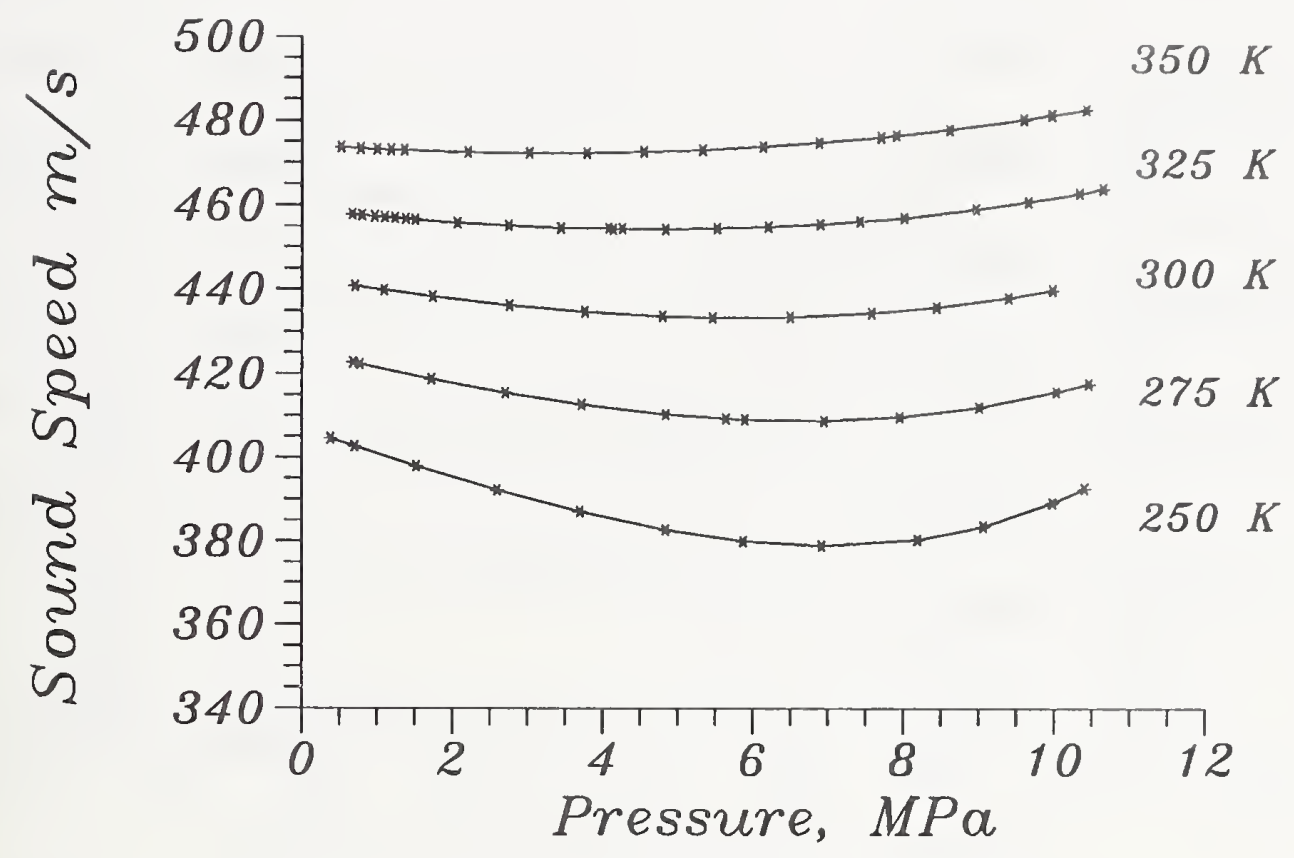

Figure 9. Experimental speed of sound for the binary mixture methane 0.95 - nitrogen 0.05 .

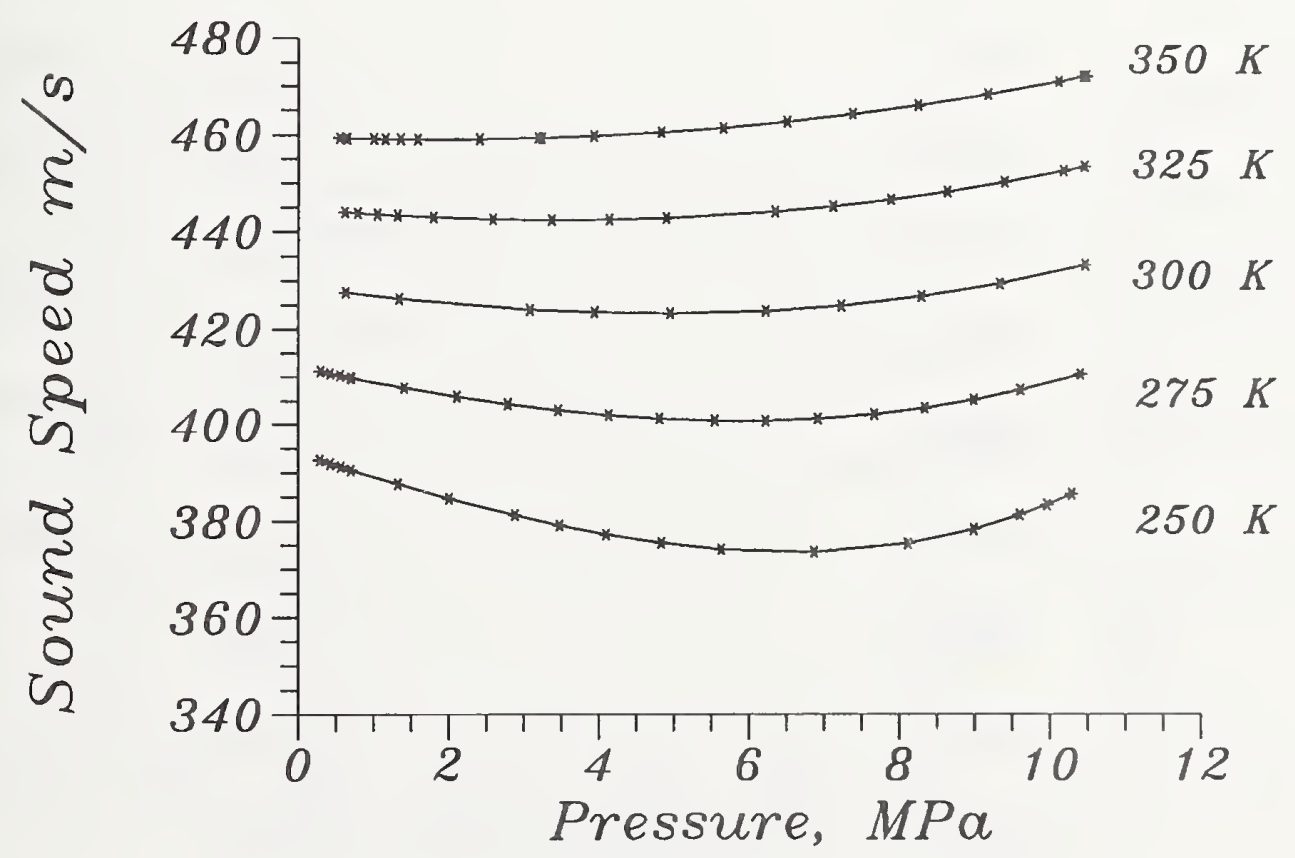

Figure 10. Experimental speed of sound for the binary mixture methane 0.85 - nitrogen 0.15 . 


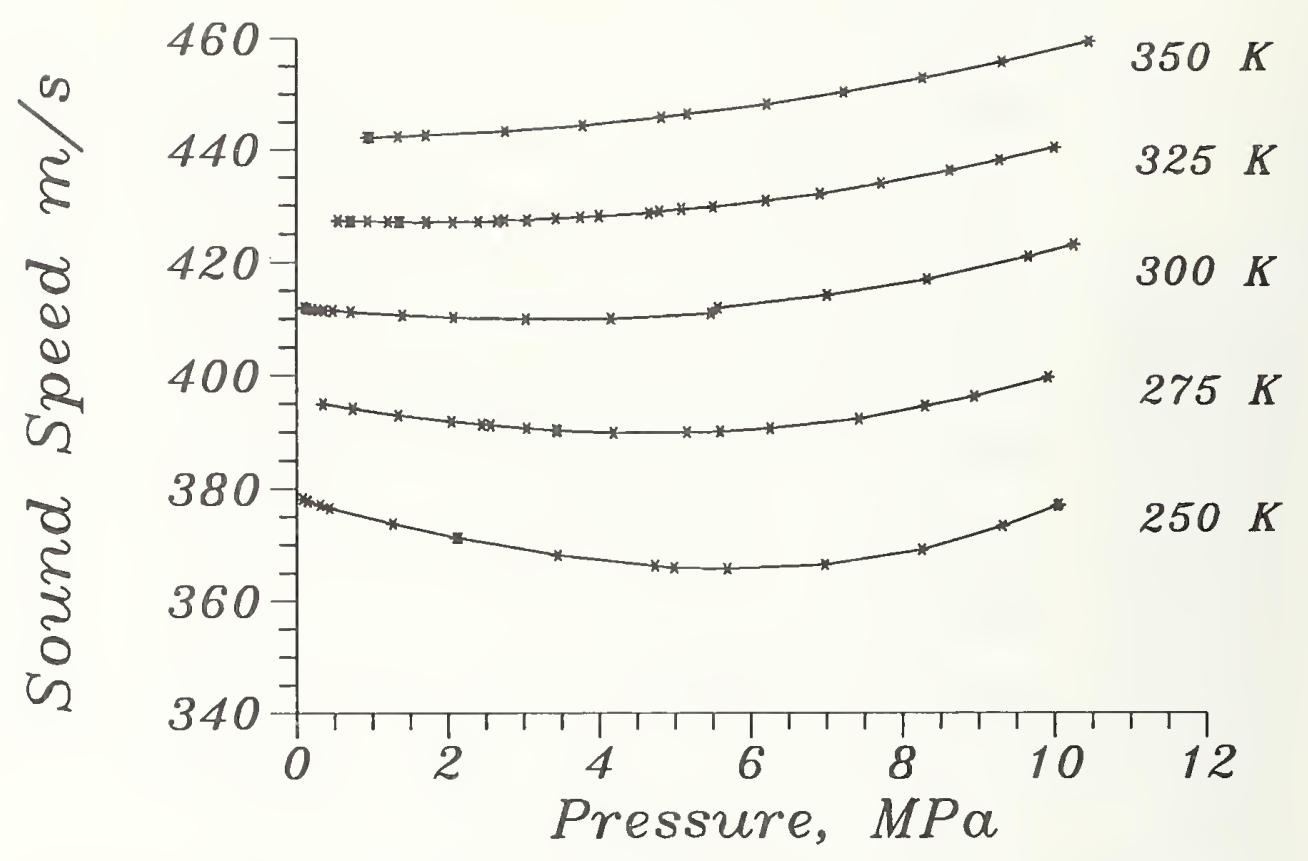

Figure 11. Experimental speed of sound for the binary mixture methane 0.71 - nitrogen 0.29.

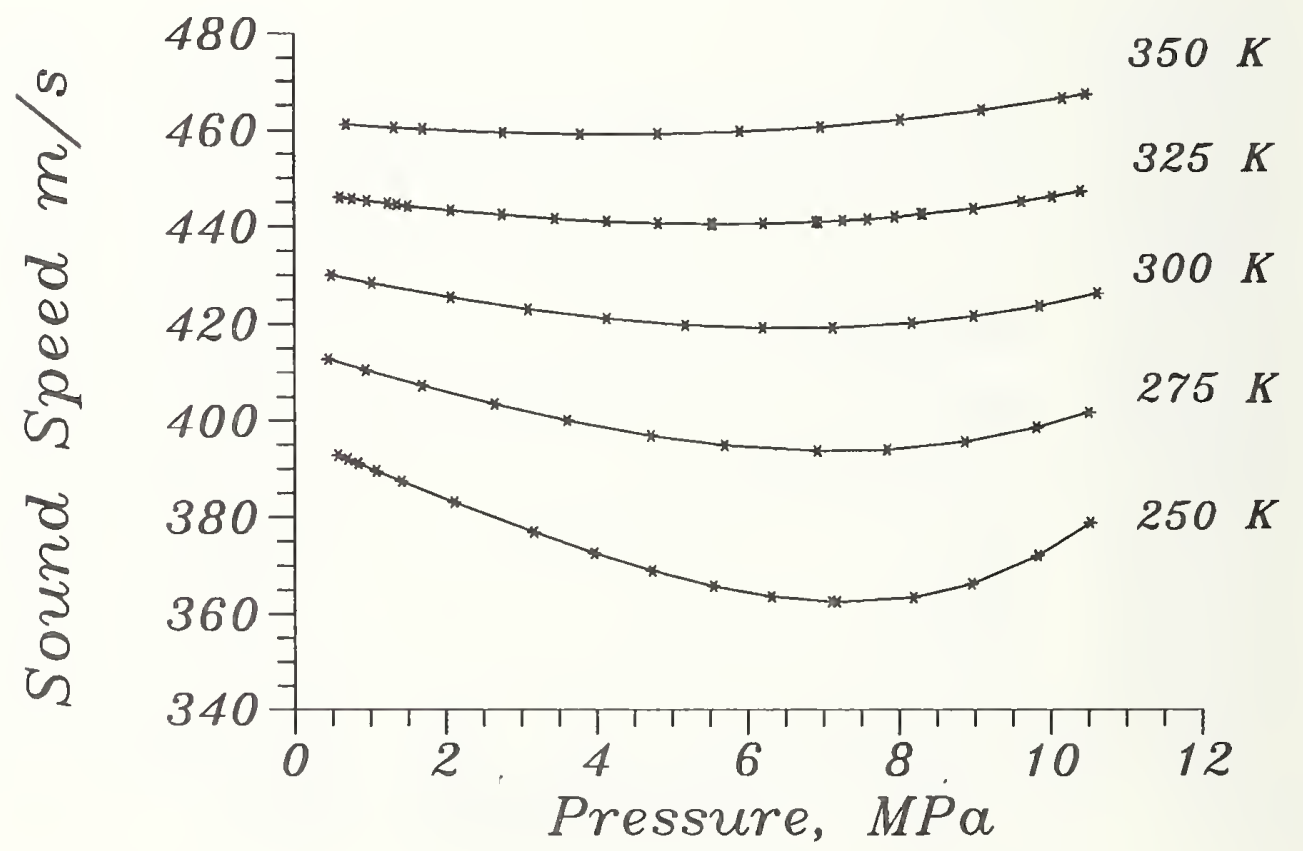

Figure 12. Experimental speed of sound for the binary mixture methane 0.95 - carbon dioxide 0.05 . 


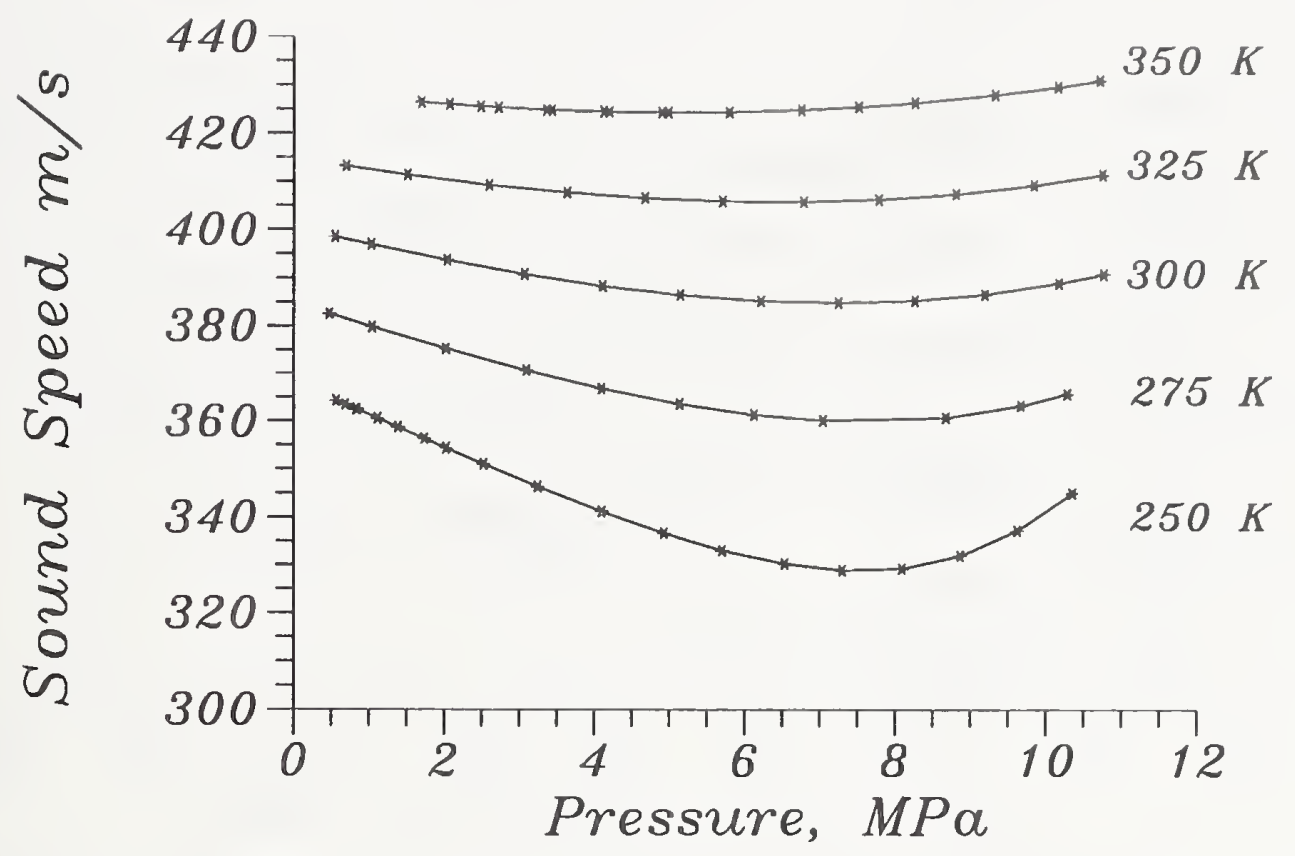

Figure 13. Experimental speed of sound for the binary mixture methane 0.85 - carbon dioxide 0.15 .

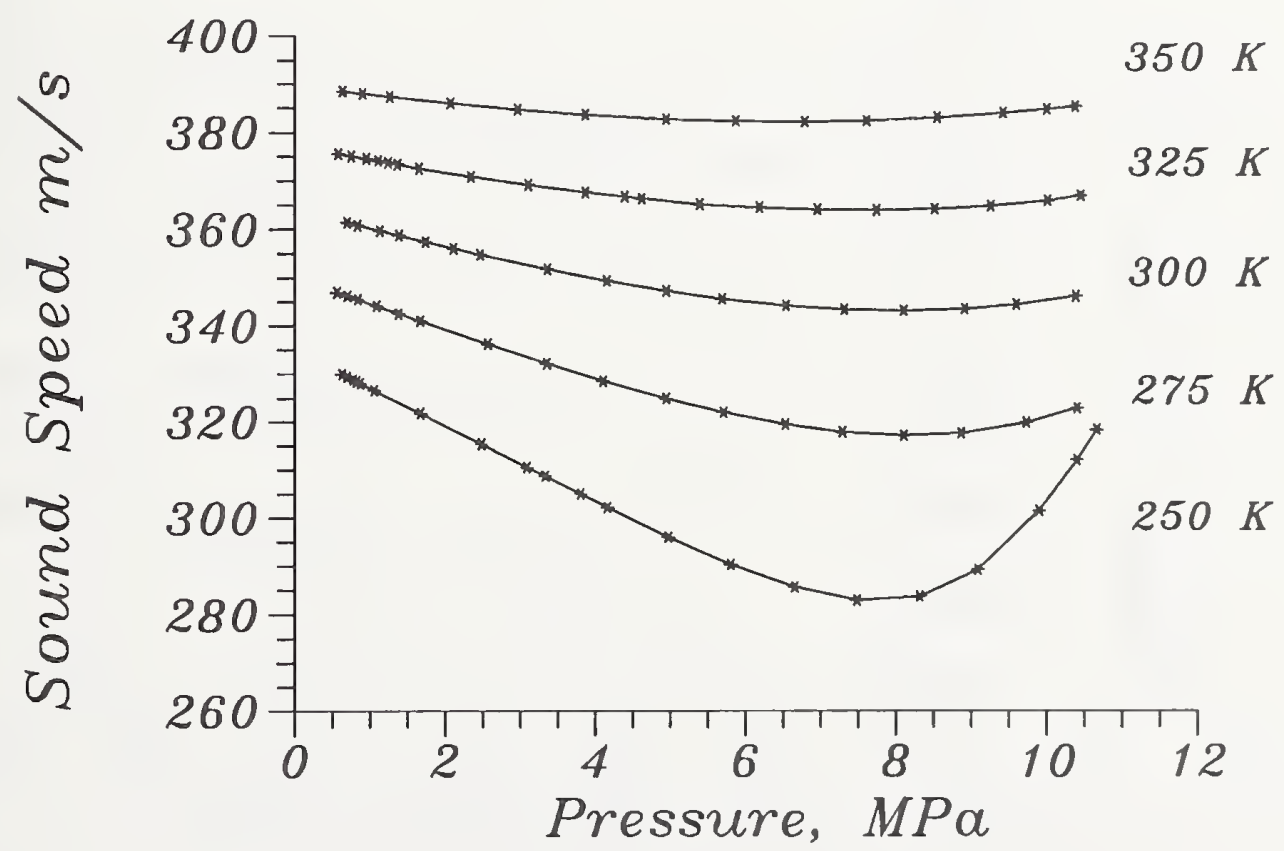

Figure 14. Experimental speed of sound for the binary mixture methane 0.70 - carbon dioxide 0.30 . 


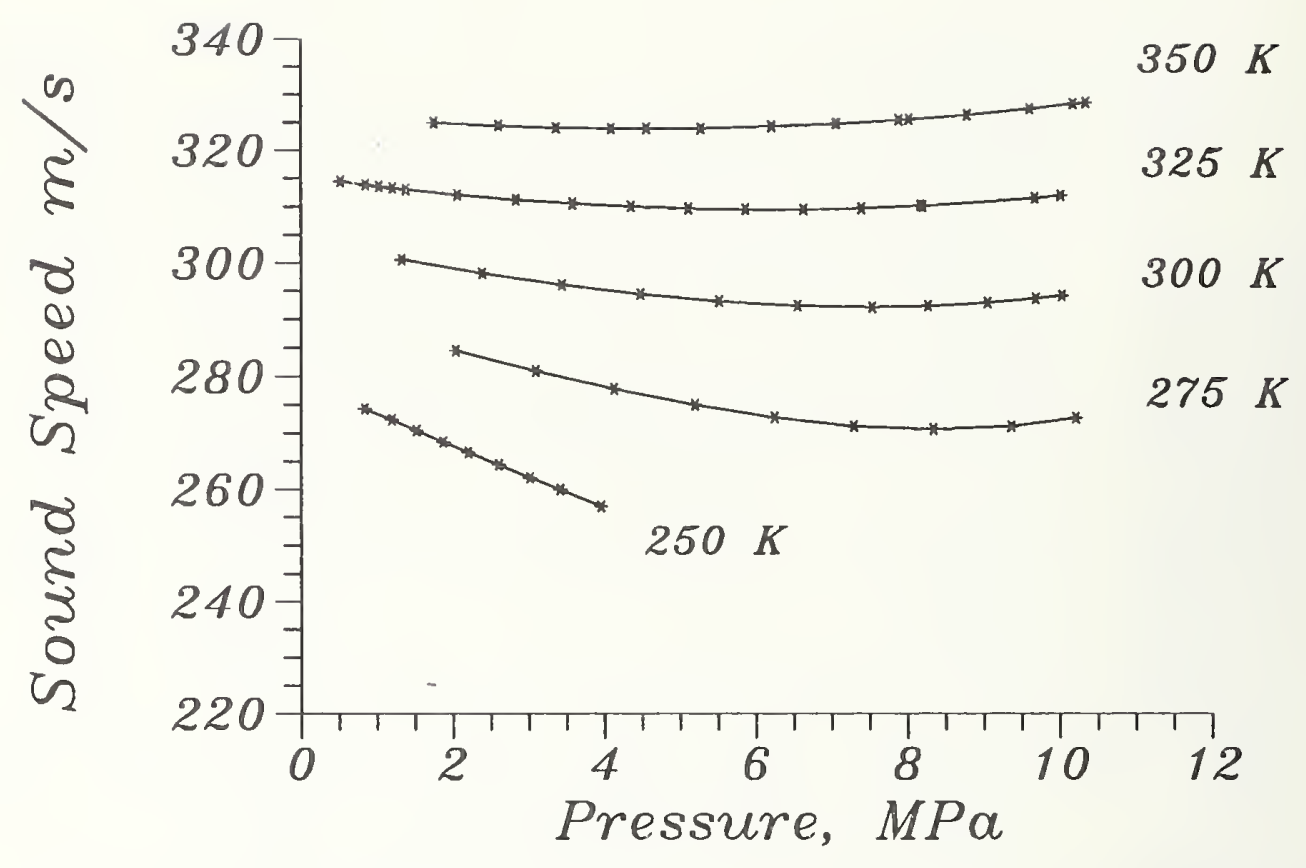

Figure 15. Experimental speed of sound for the binary mixture nitrogen 0.50 - carbon dioxide 0.50 .

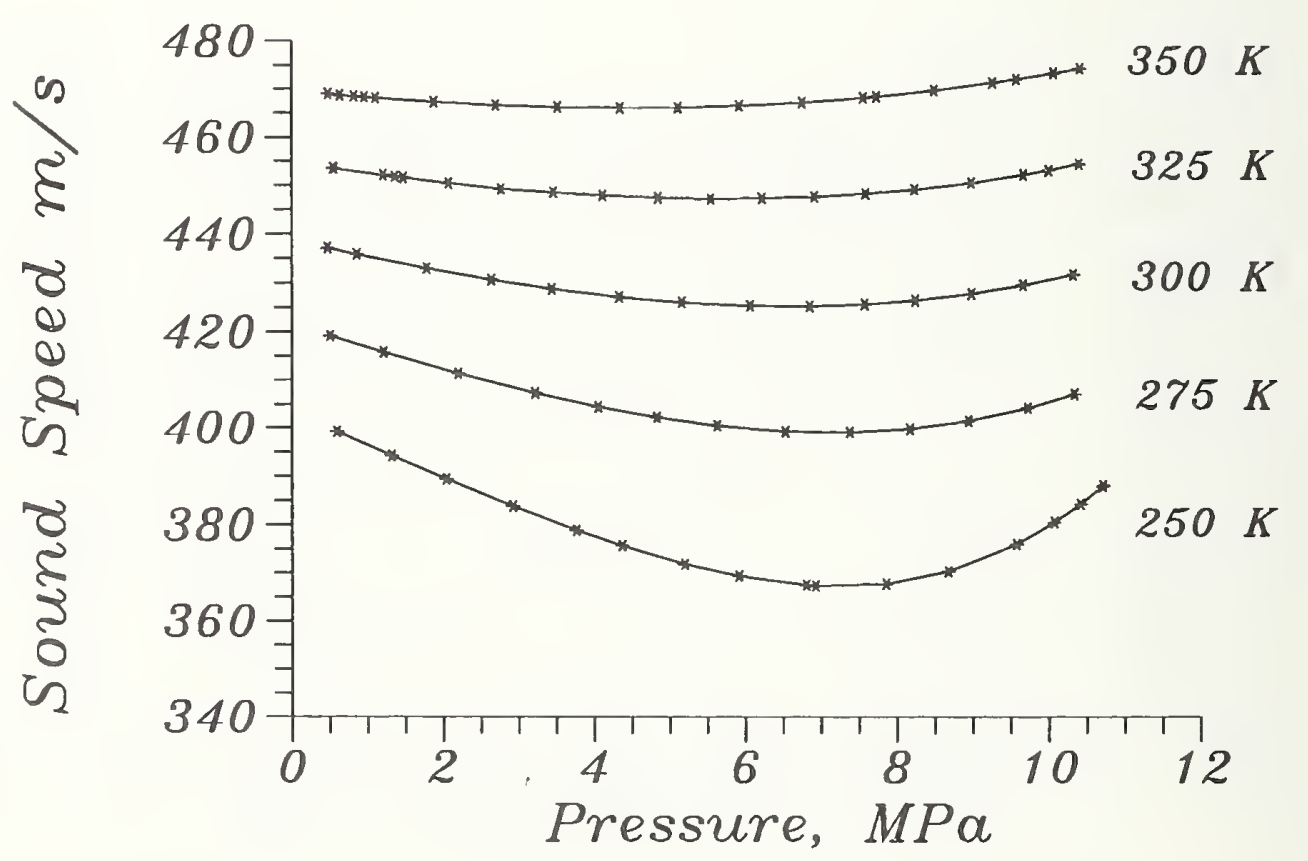

Figure 16. Experimental speed of sound for the Gulf Coast mixture. 


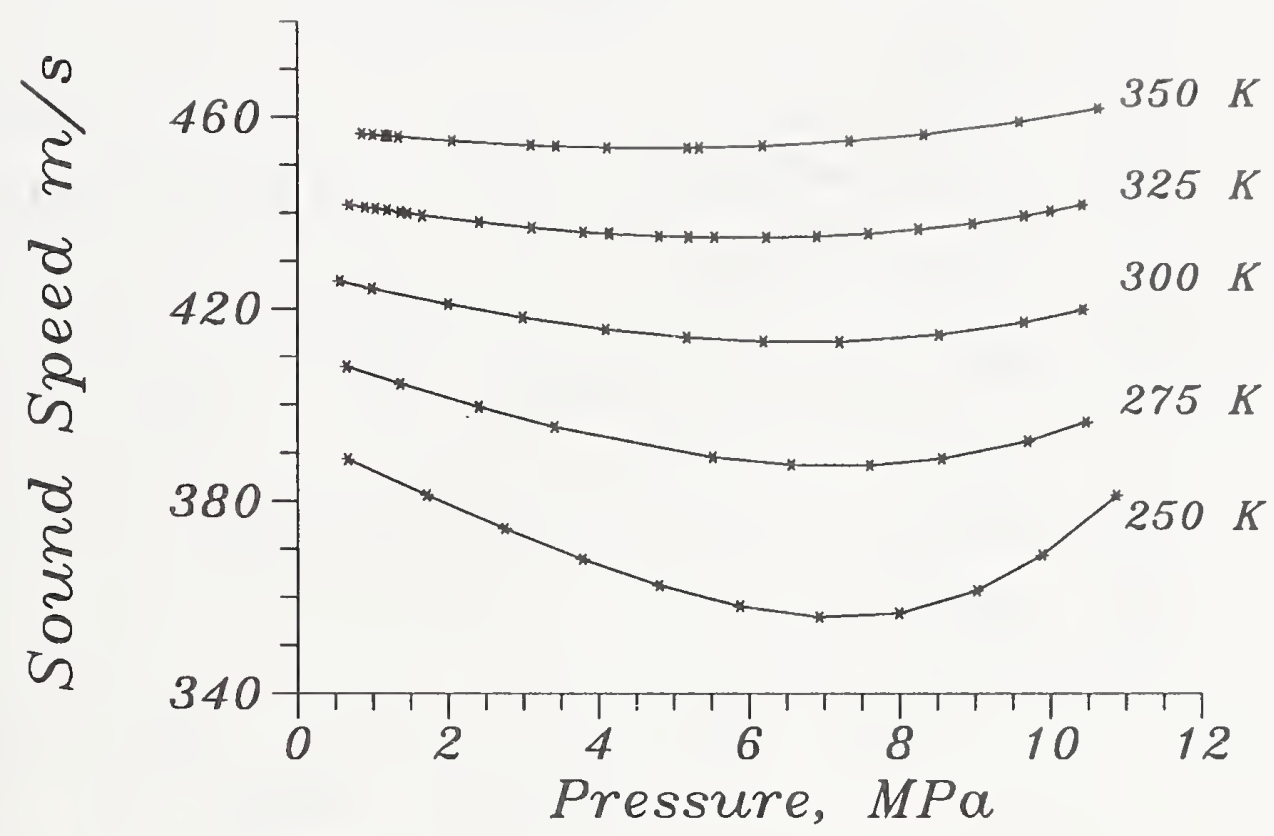

Figure 17. Experimental speed of sound for the Amarillo mixture.

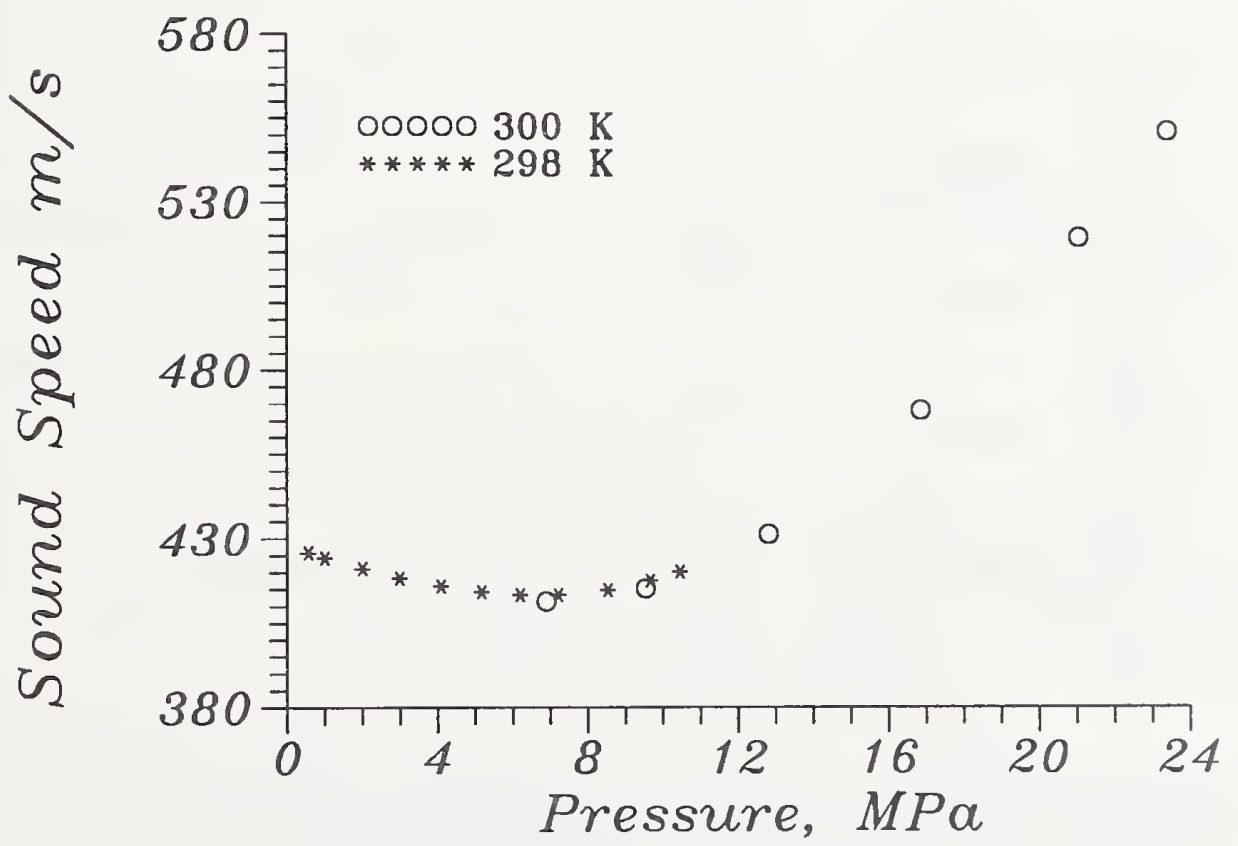

Figure 18. Experimental speed of sound for the Amarillo mixture. Comparing the $300 \mathrm{~K}$ mun to the high pressure isotherm at $298 \mathrm{~K}$. 


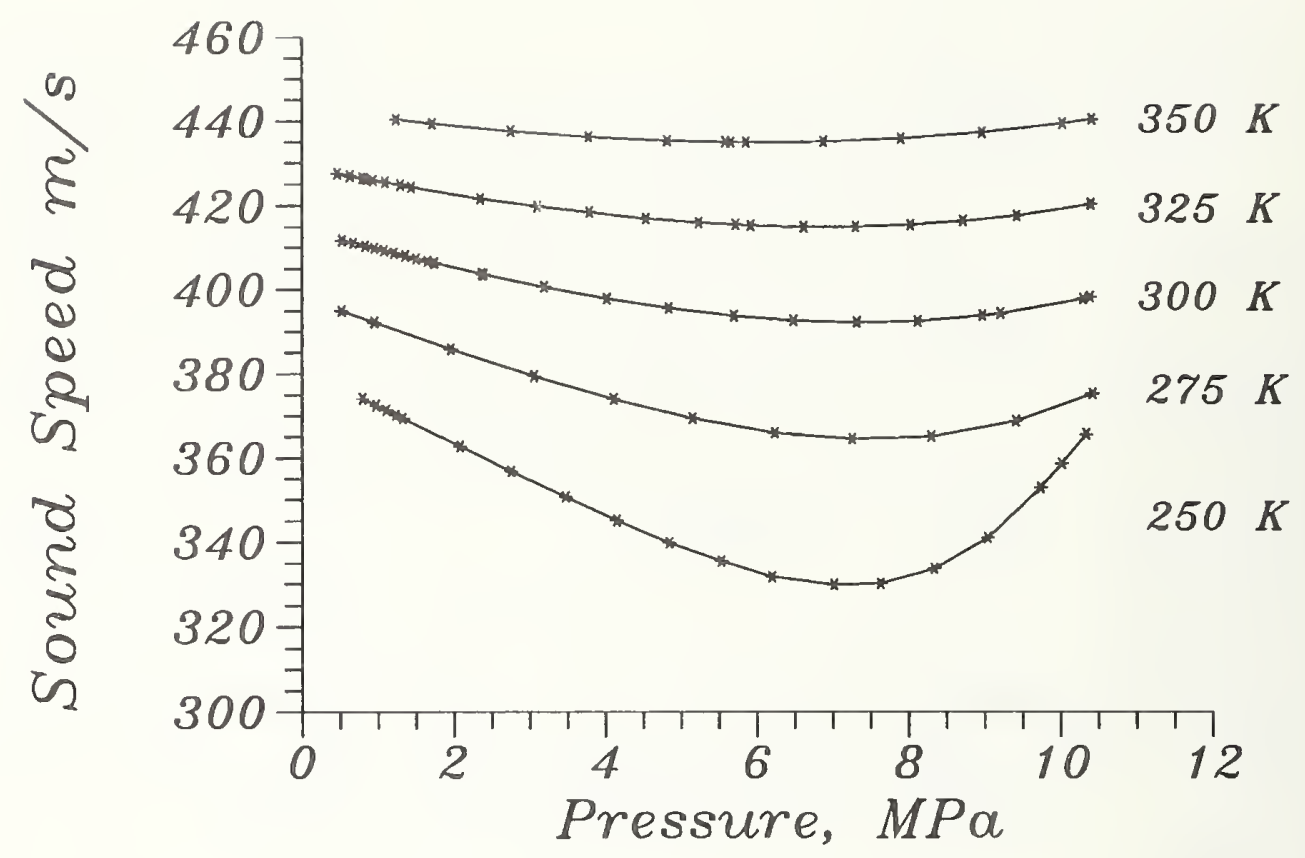

Figure 19. Experimental speed of sound for the Statoil dry gas mixture.

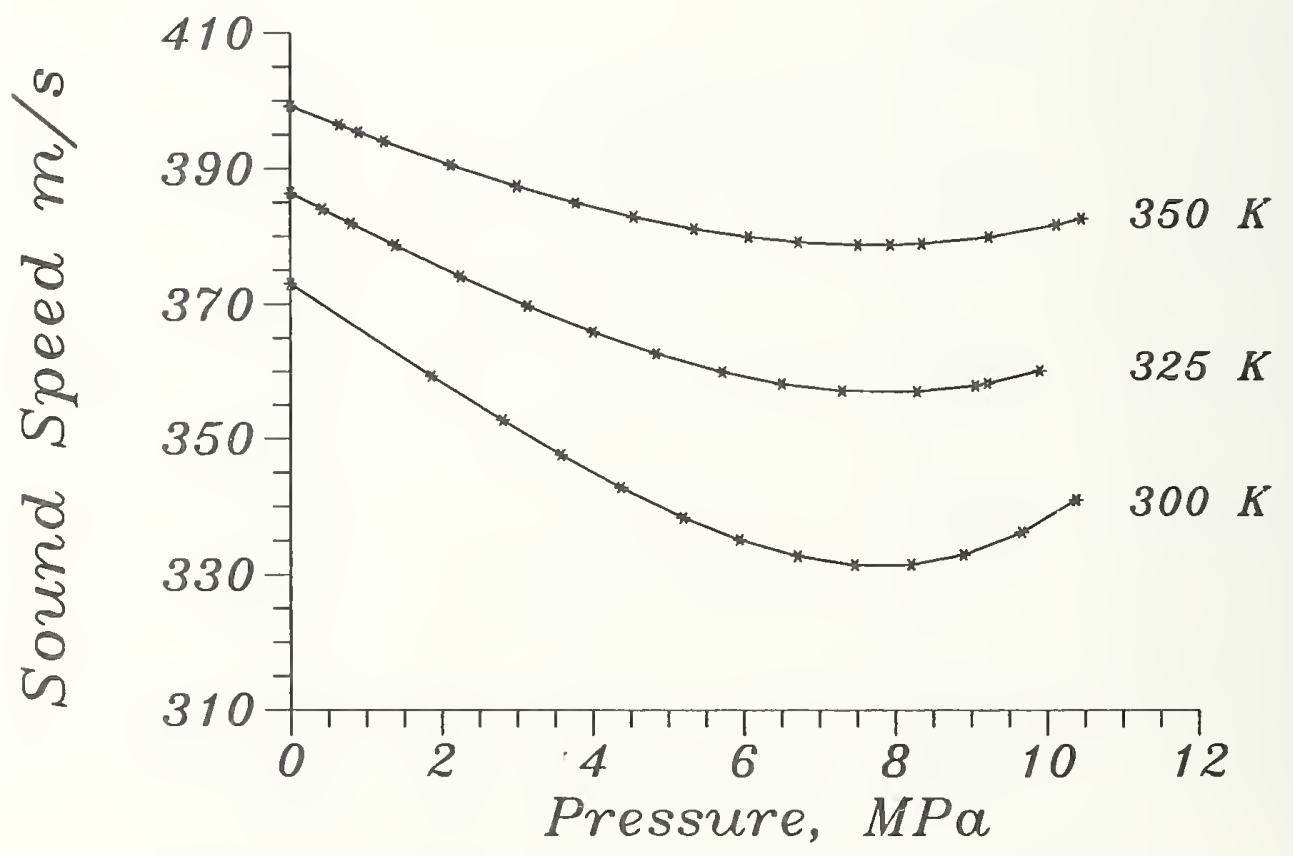

Figure 20. Experimental speed of sound for the Statoil Statvordgass mixture. 


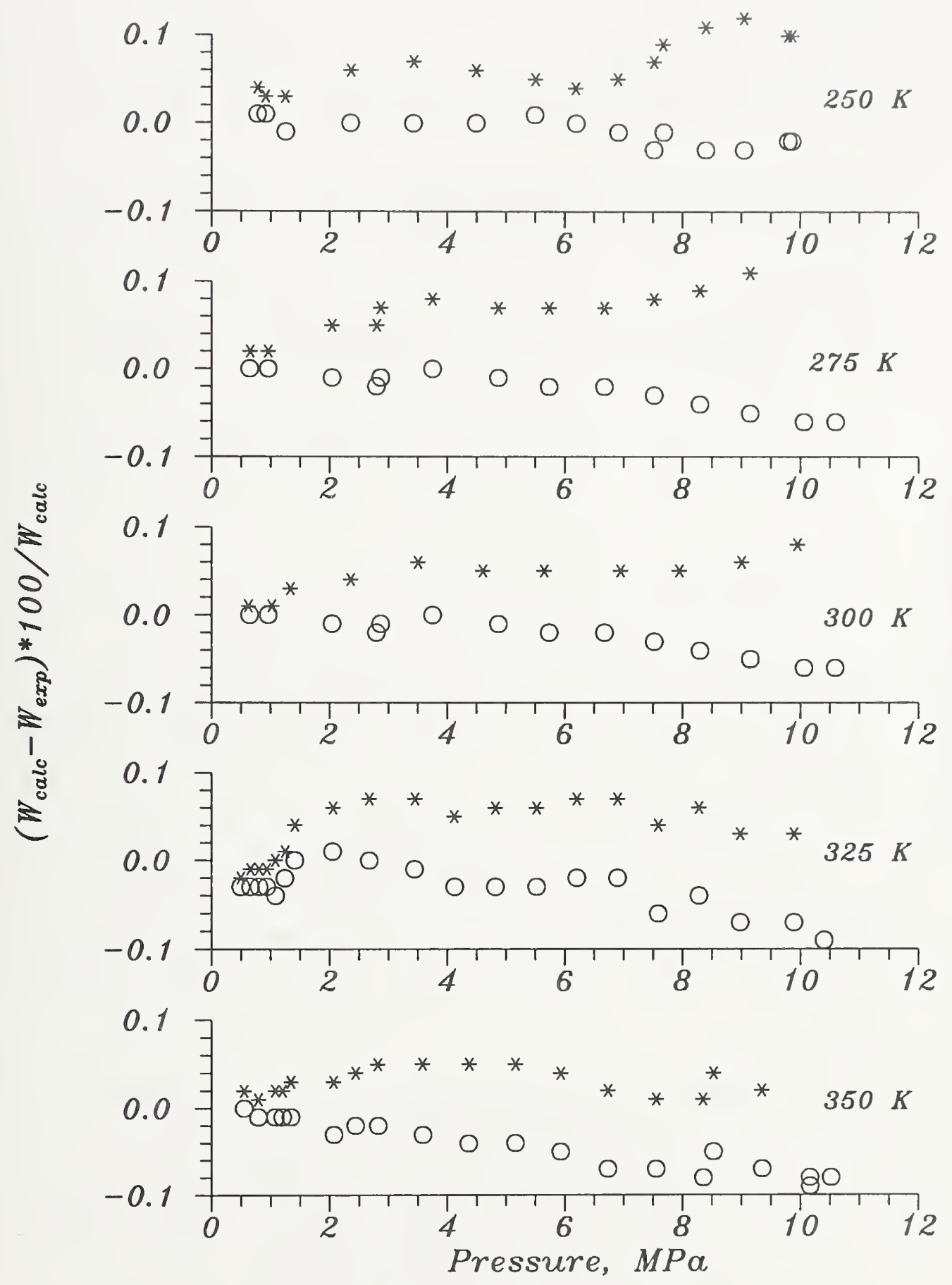

Figure 21. Deviations of speed of sound computed by AGA 8 and NGAS from the experimental values for the binary mixture, methane 0.95 ethane 0.05 . 


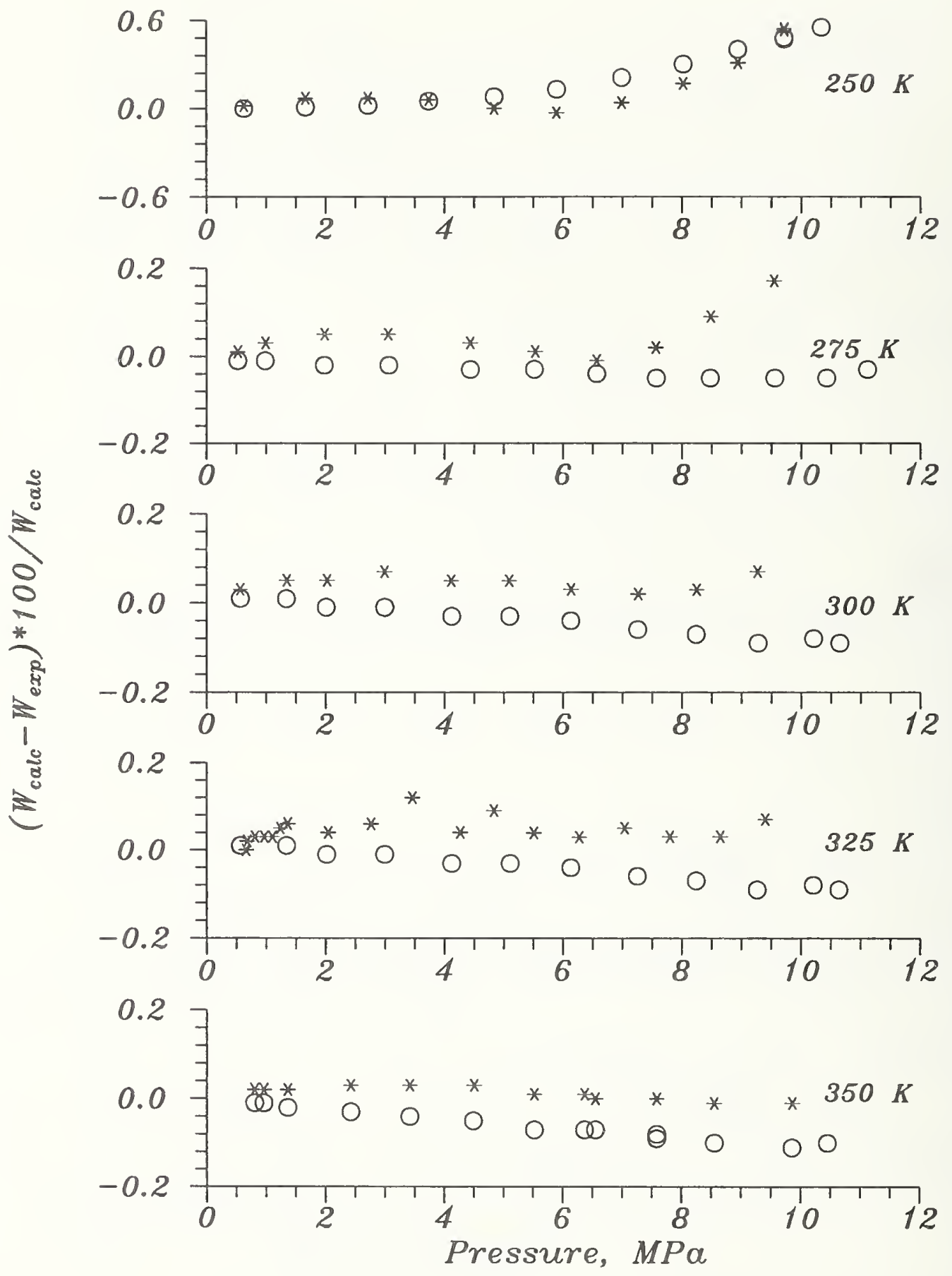

Figure 22. Deviations of speed of sound computed by $A G A 8$ and NGAS from the experimental values for the binary mixture, methane 0.85 - ethane 0.15 . 


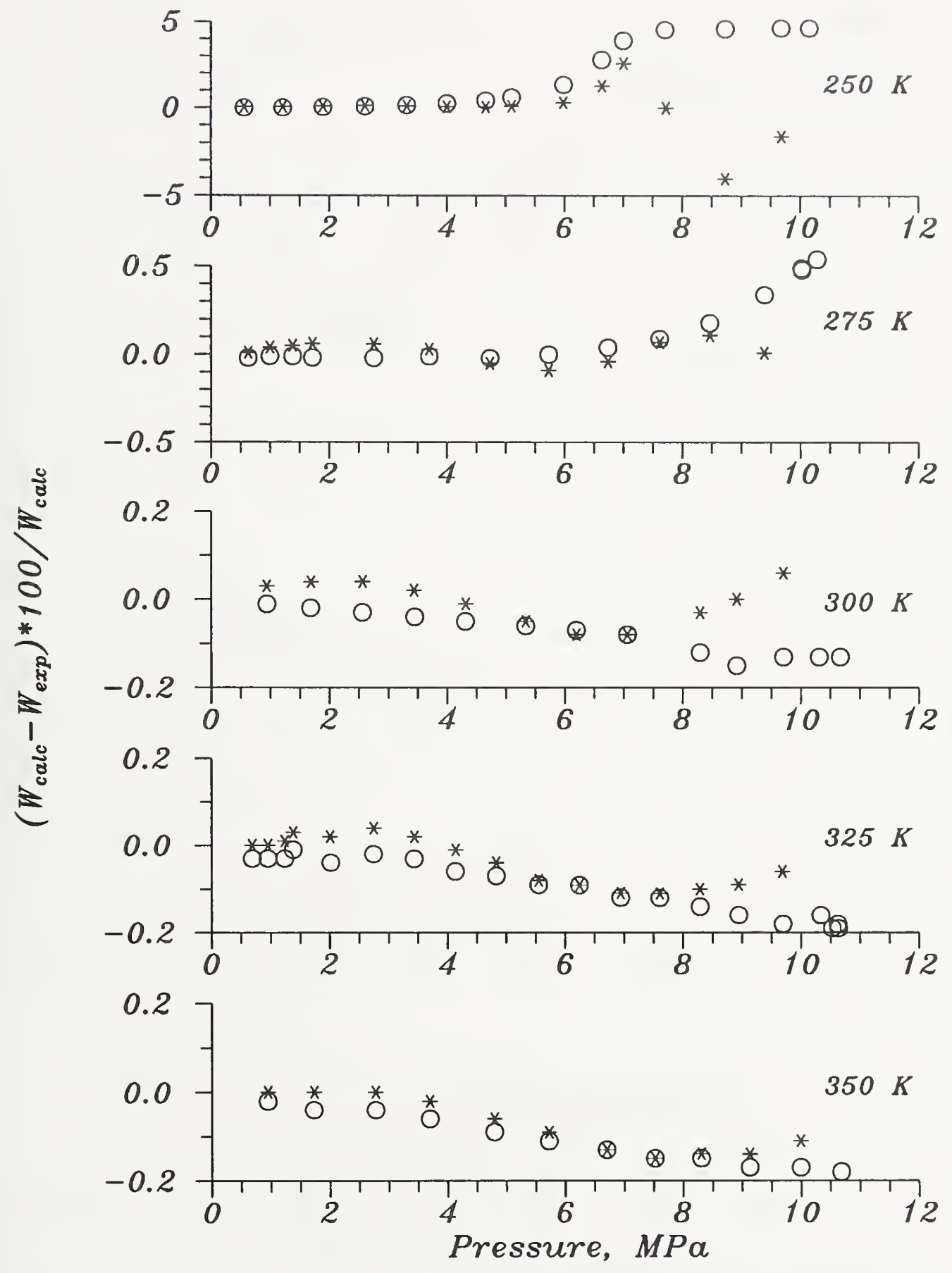

Figure 23. Deviations of speed of sound computed by $A G A 8$ and NGAS from the experimental values for the binary mixture, methane 0.69 - ethane 0.31 . 


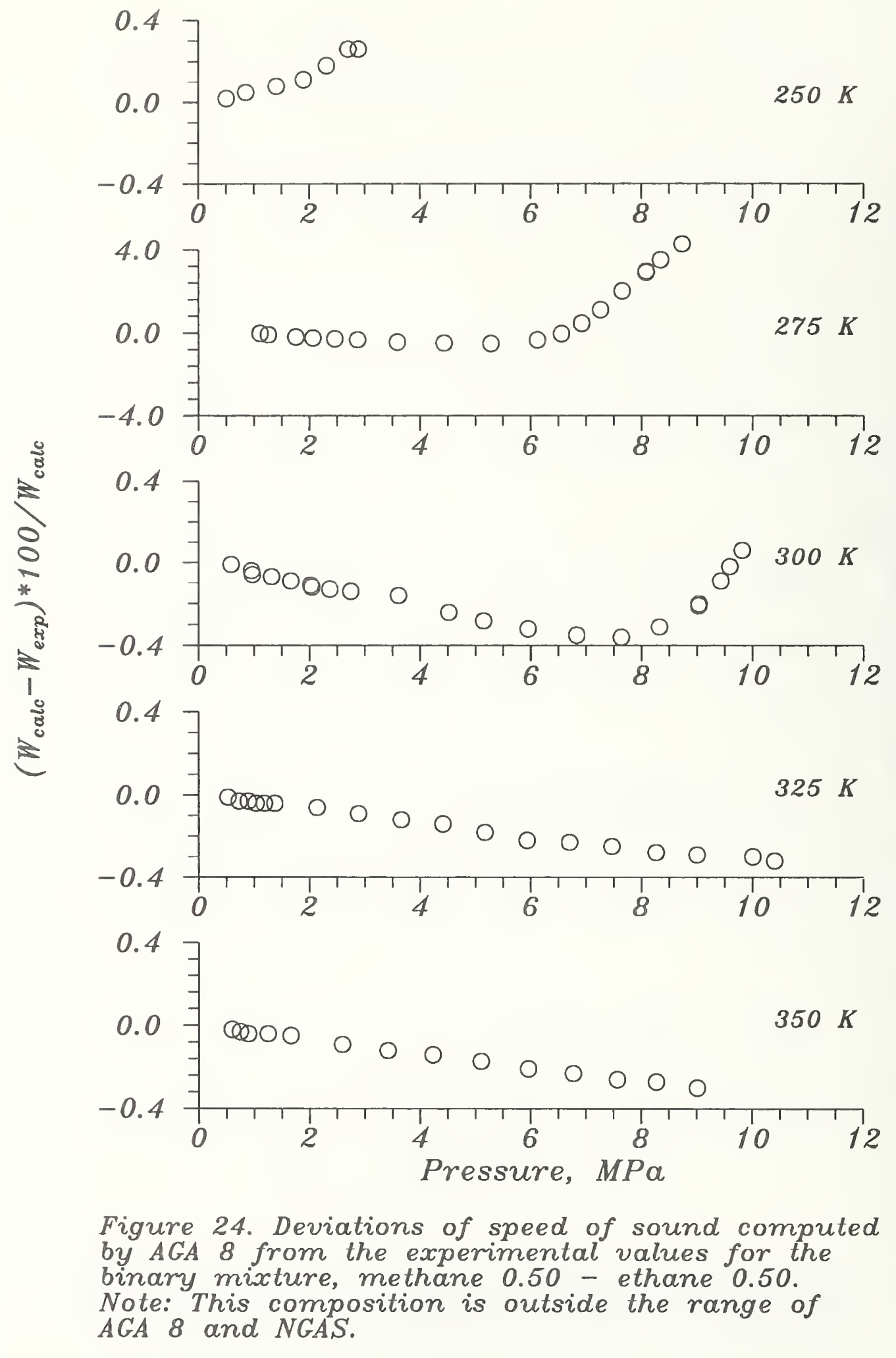




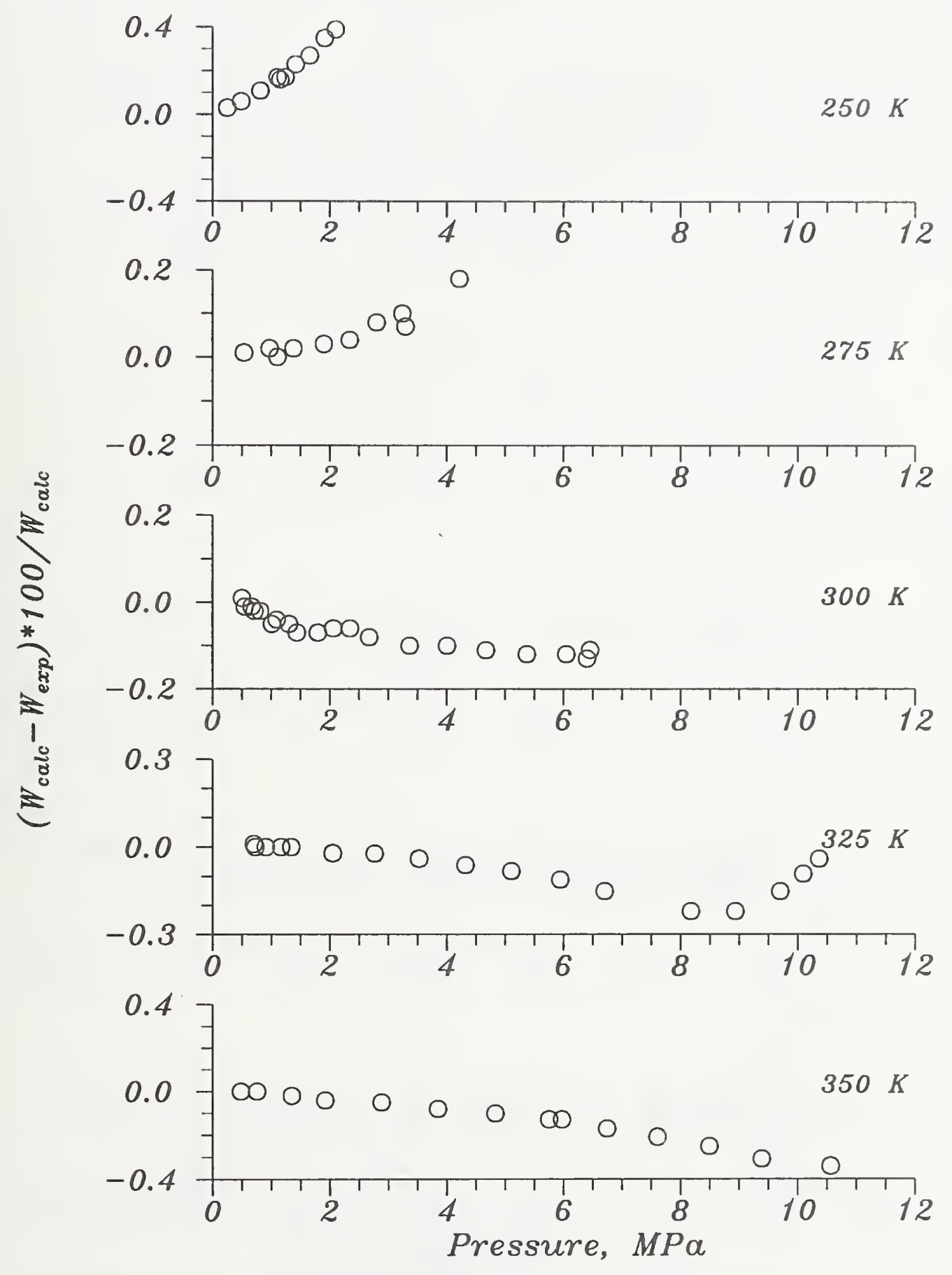

Figure 25. Deviations of speed of sound computed by $A G A 8$ from the experimental values for the binary mixture, methane 0.35 - ethane 0.65 . NOTE: This composition is outside the range of $A G A \&$ and NGAS. 


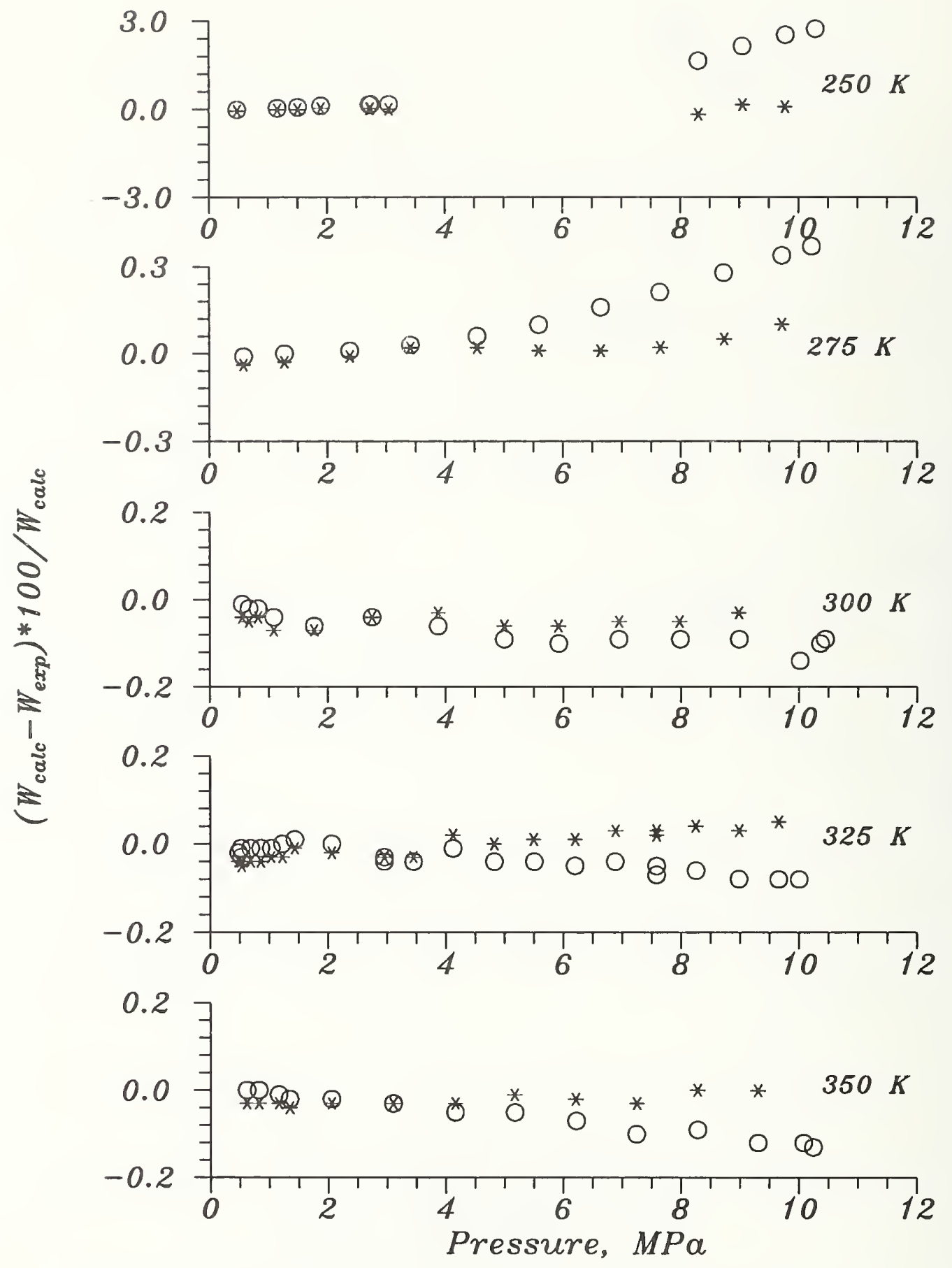

Figure 26. Deviations of speed of sound computed by $A G A 8$ and NGAS from the experimental values for the binary mixture, methane 0.90 - propane 0.10 .

NOTE: This composition is outside the range of $A G A \&$ and NGAS. 


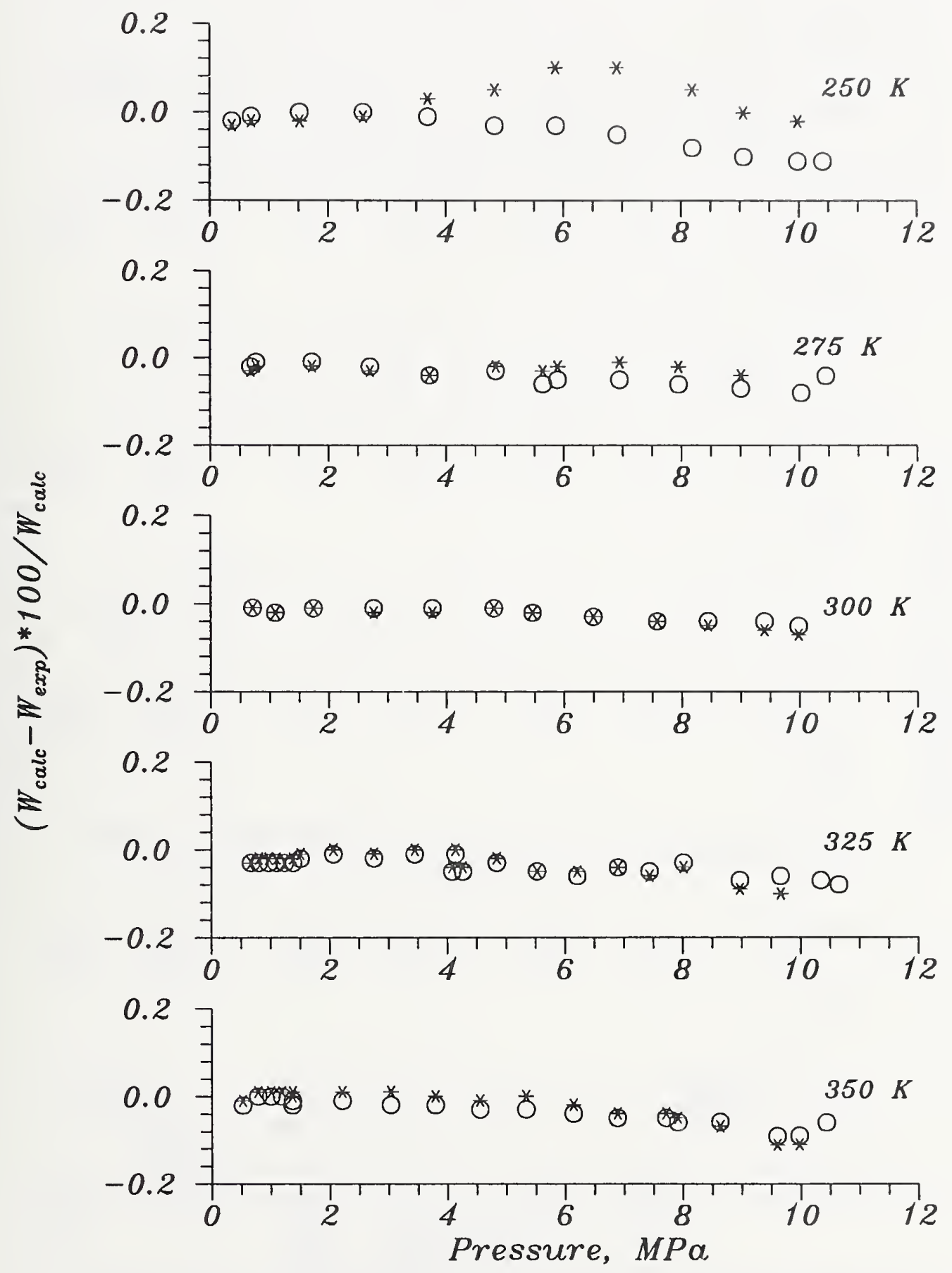

Figure 27. Deviations of speed of sound computed by $A G A 8$ and NGAS from the experimental values for the binary mixture, methane 0.95 - nitrogen 0.05 . 


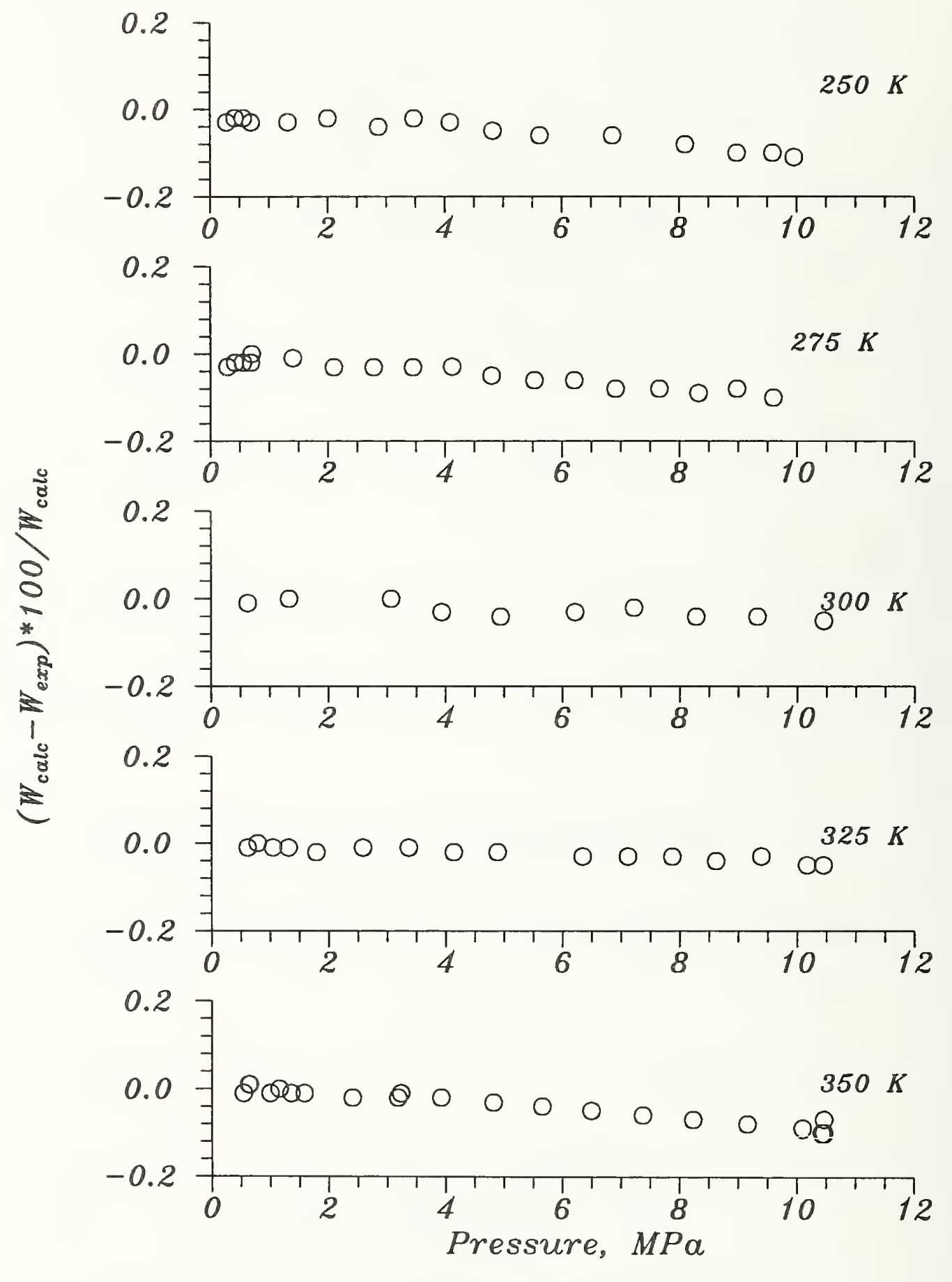

Figure 28. Deviations of speed of sound computed by $A G A 8$ from the experimental values for the binary mixture, methane 0.85 - nitrogen 0.15 . NOTE: This composition is outside the range of NGAS. 


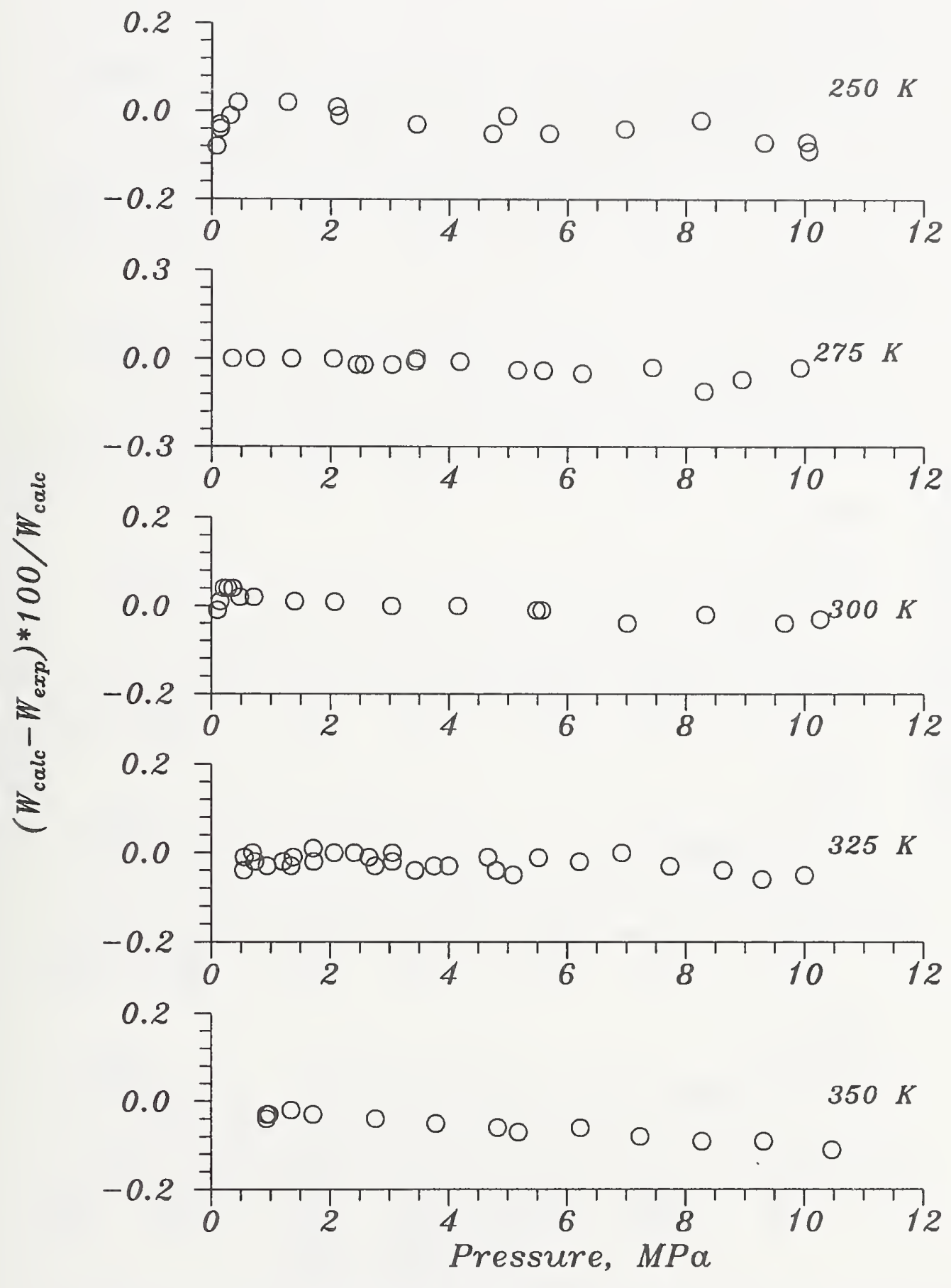

Figure 29. Deviations of speed of sound computed by $A G A 8$ from the experimental values for the binary mixture, methane 0.71 - nitrogen 0.29. NOTE: This composition is outside the range of NGAS. 


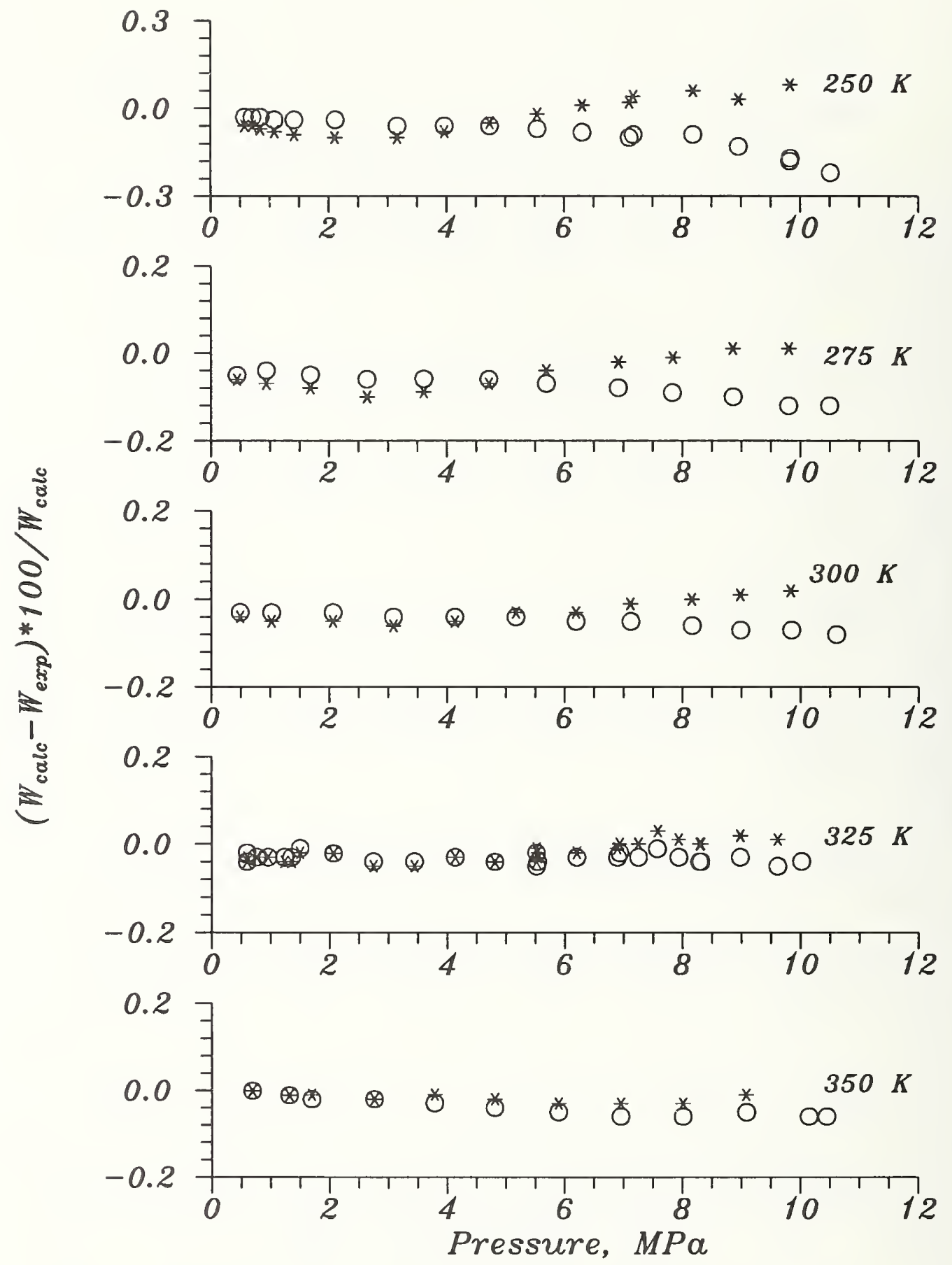

Figure 30. Deviations of speed of sound computed by $A G A 8$ and NGAS from the experimental values for the binary mixture, methane 0.95 carbon dioxide 0.05 . 


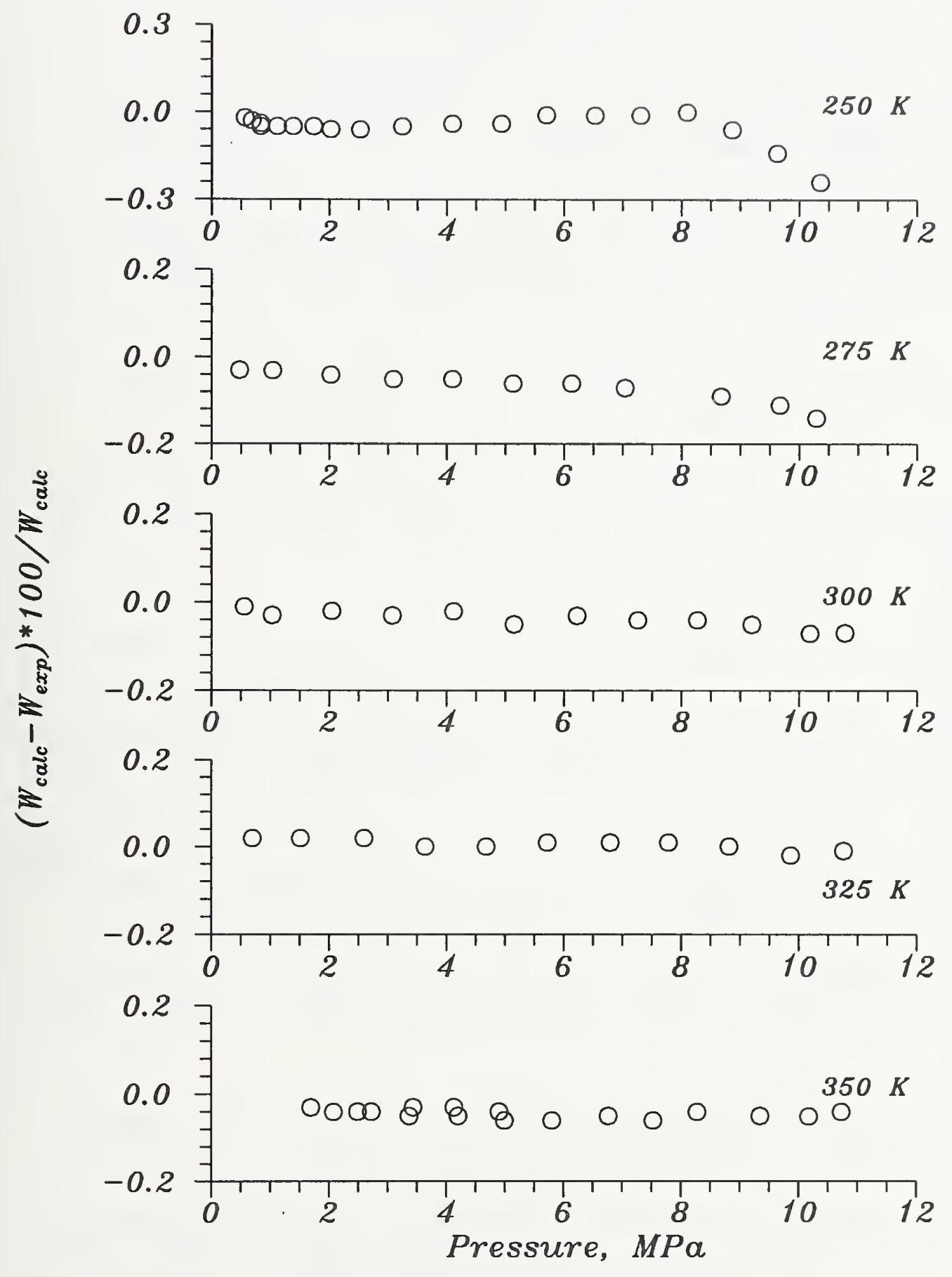

Figure 31. Deviations of speed of sound computed by $A G A B$ and NGAS from the experimental values for the binary mixture, methane 0.85 - carbon dioxide 0.15 . NOTE: This composition is outside the range of NGAS. 


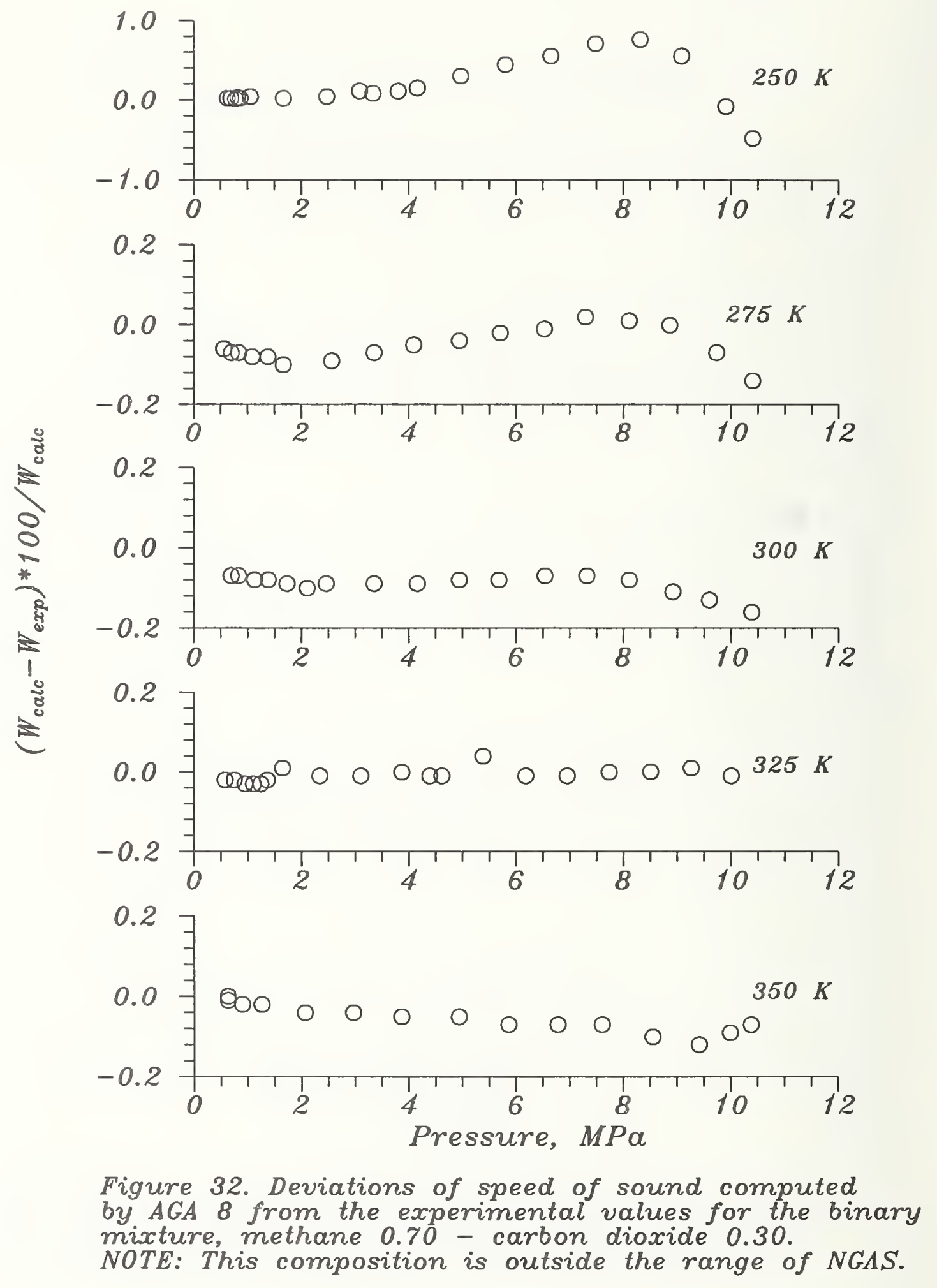




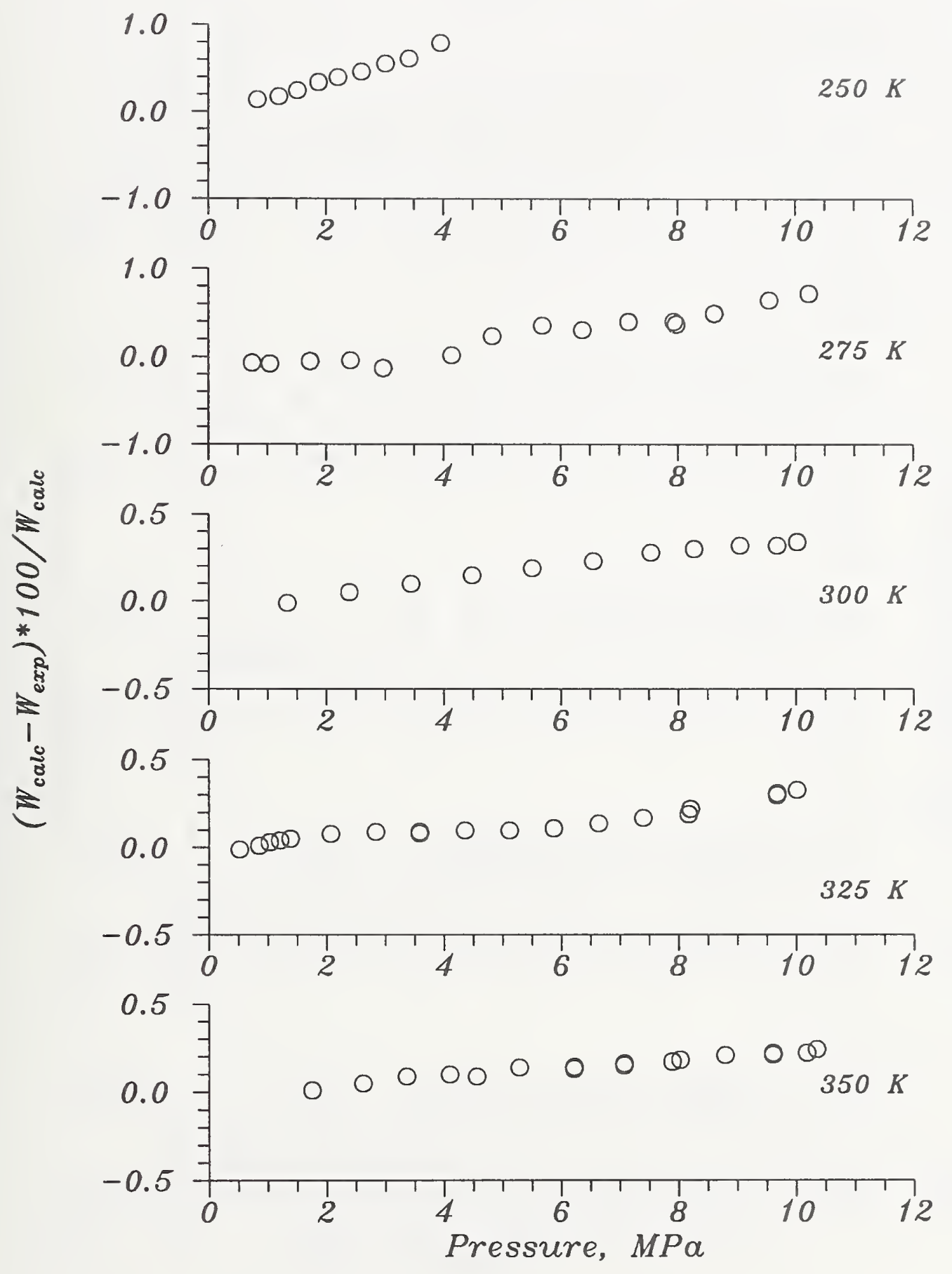

Figure 33. Deviations of speed of sound computed by $A G A 8$ from the experimental values for the binary mixture, nitrogen 0.50 - carbon dioxide 0.50 . NOTE: This composition is outside the range of $A G A 8$ and NGAS. 


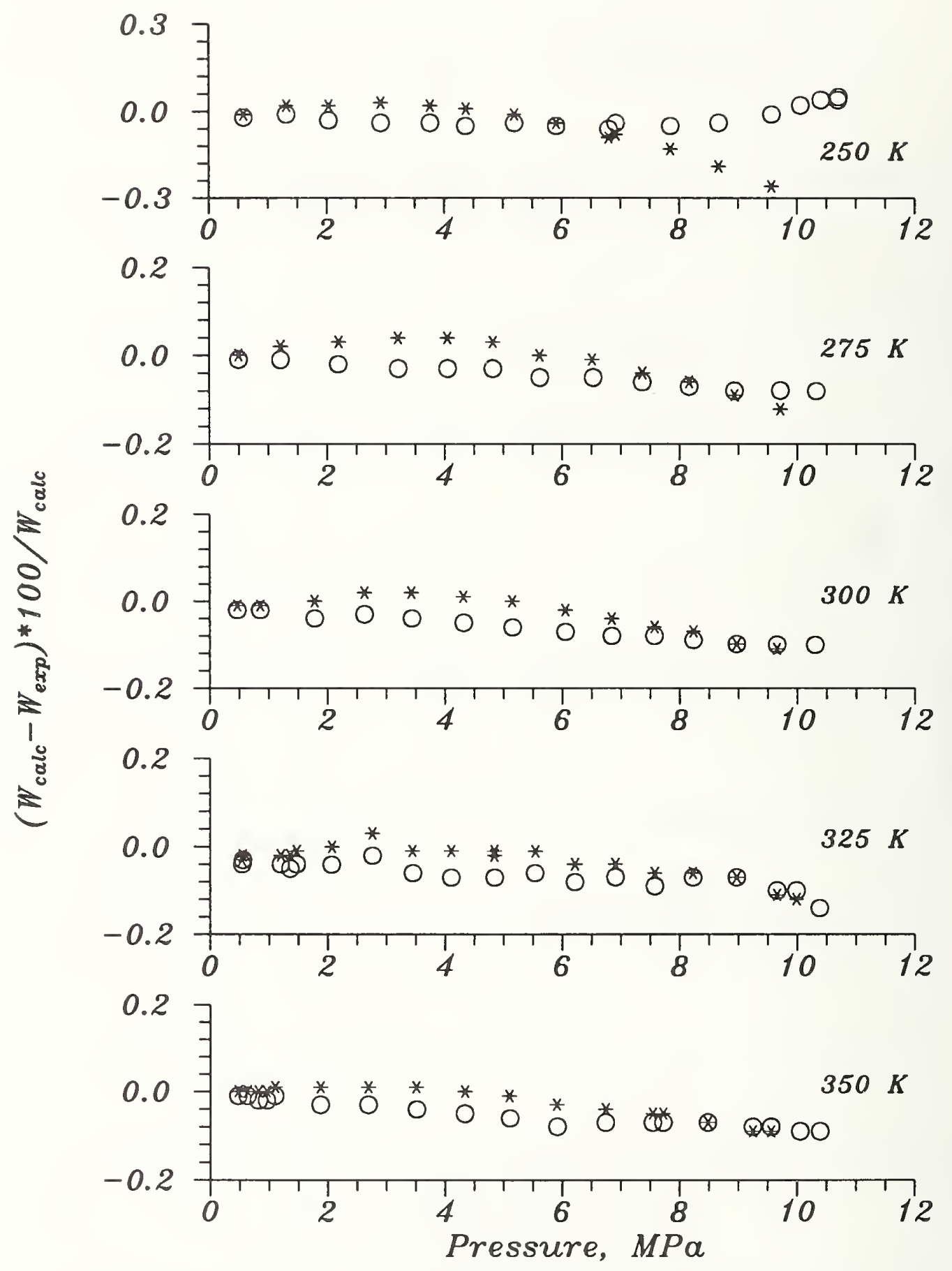

Figure 34. Deviations of speed of sound computed by AGA 8 and NGAS from the experimental values for the Gulf Coast mixture. 


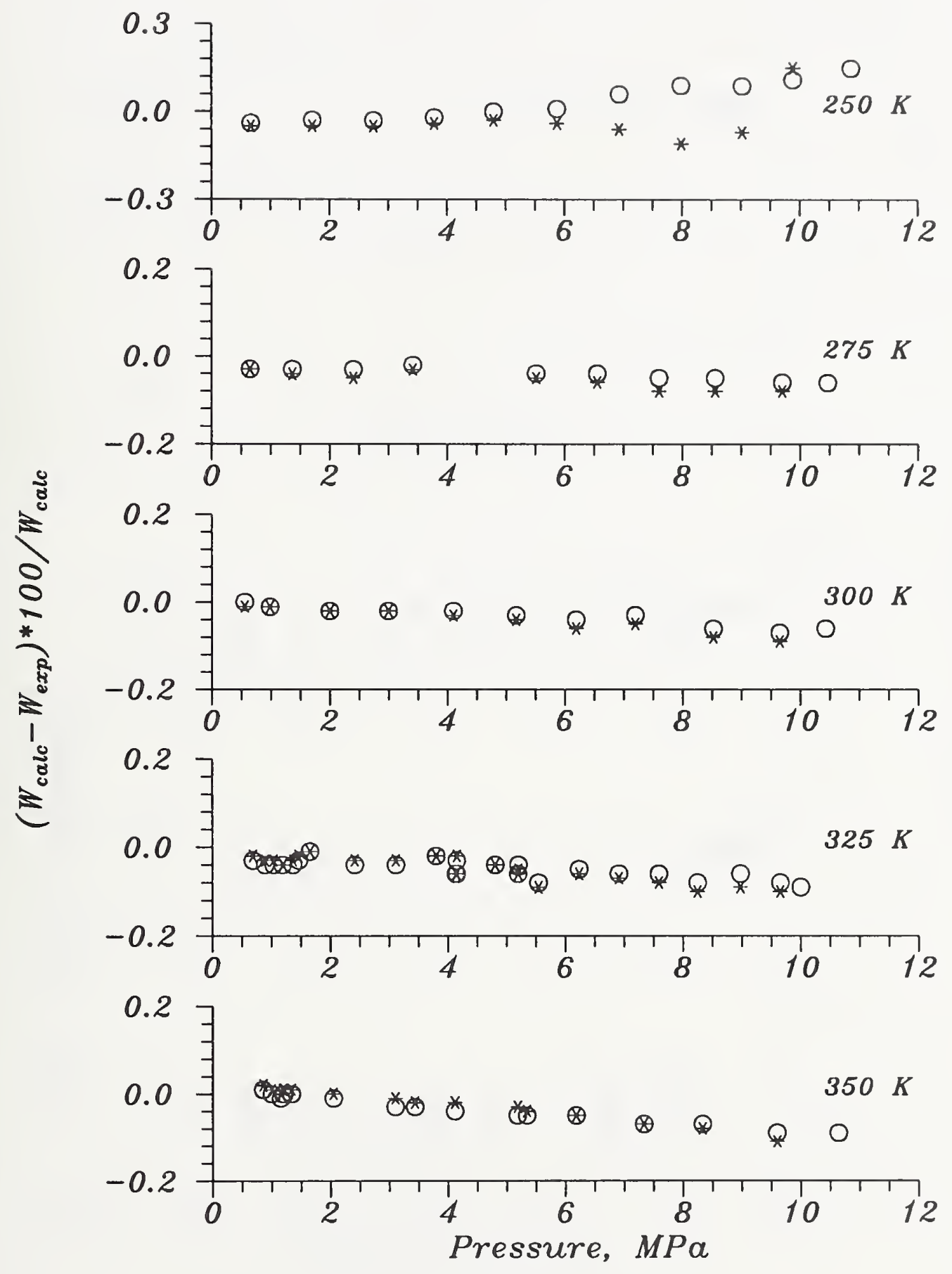

Figure 35. Deviations of speed of sound computed by $A G A 8$ and NGAS from the experimental values for the Amarillo mixture. 


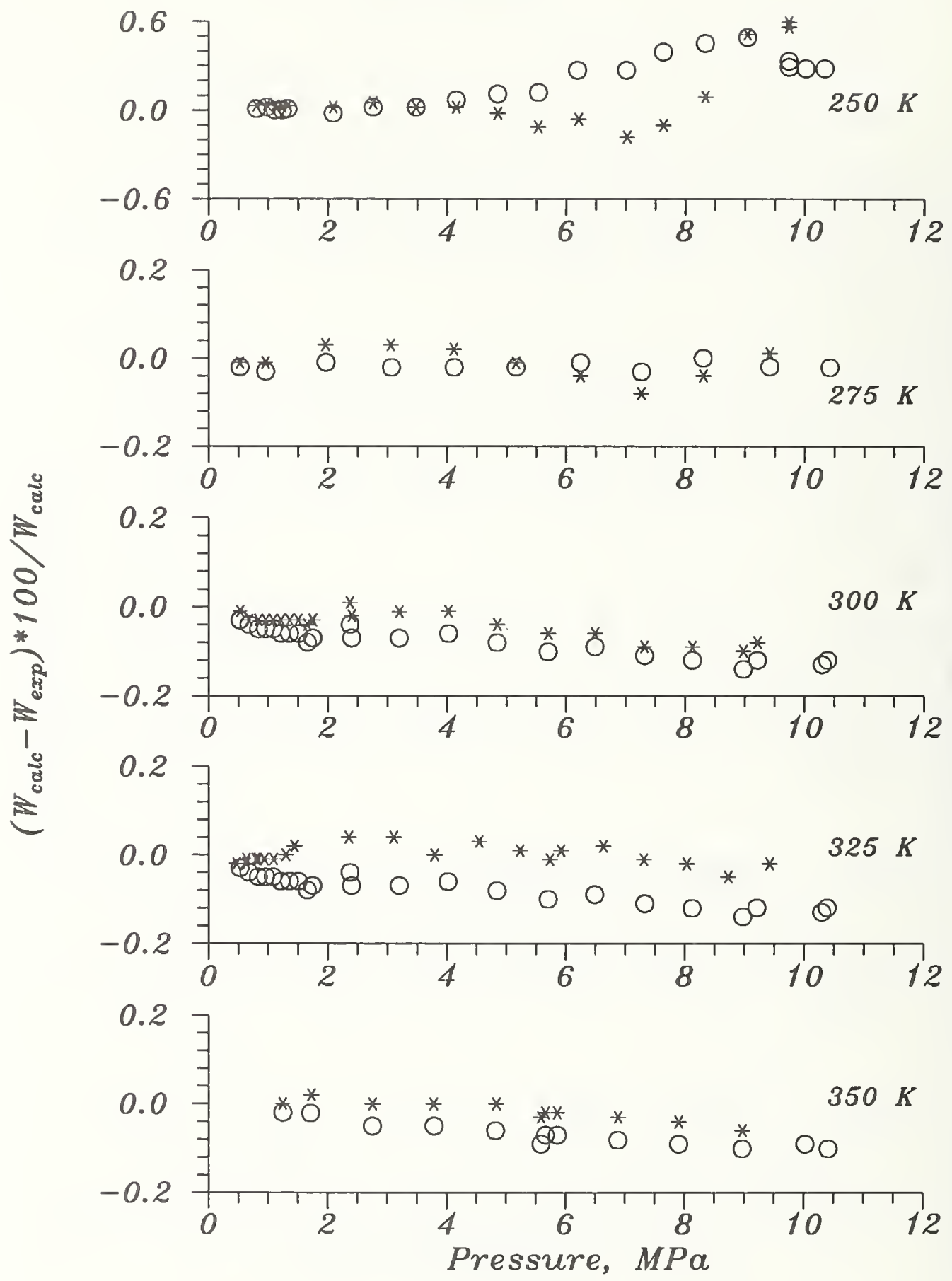

Figure 36. Deviations of speed of sound computed by AGA 8 and NGAS from the experimental values for the Statoil dry gas mixture. 
00000 AGA 8

$* * * * * \mathrm{NGAS}$

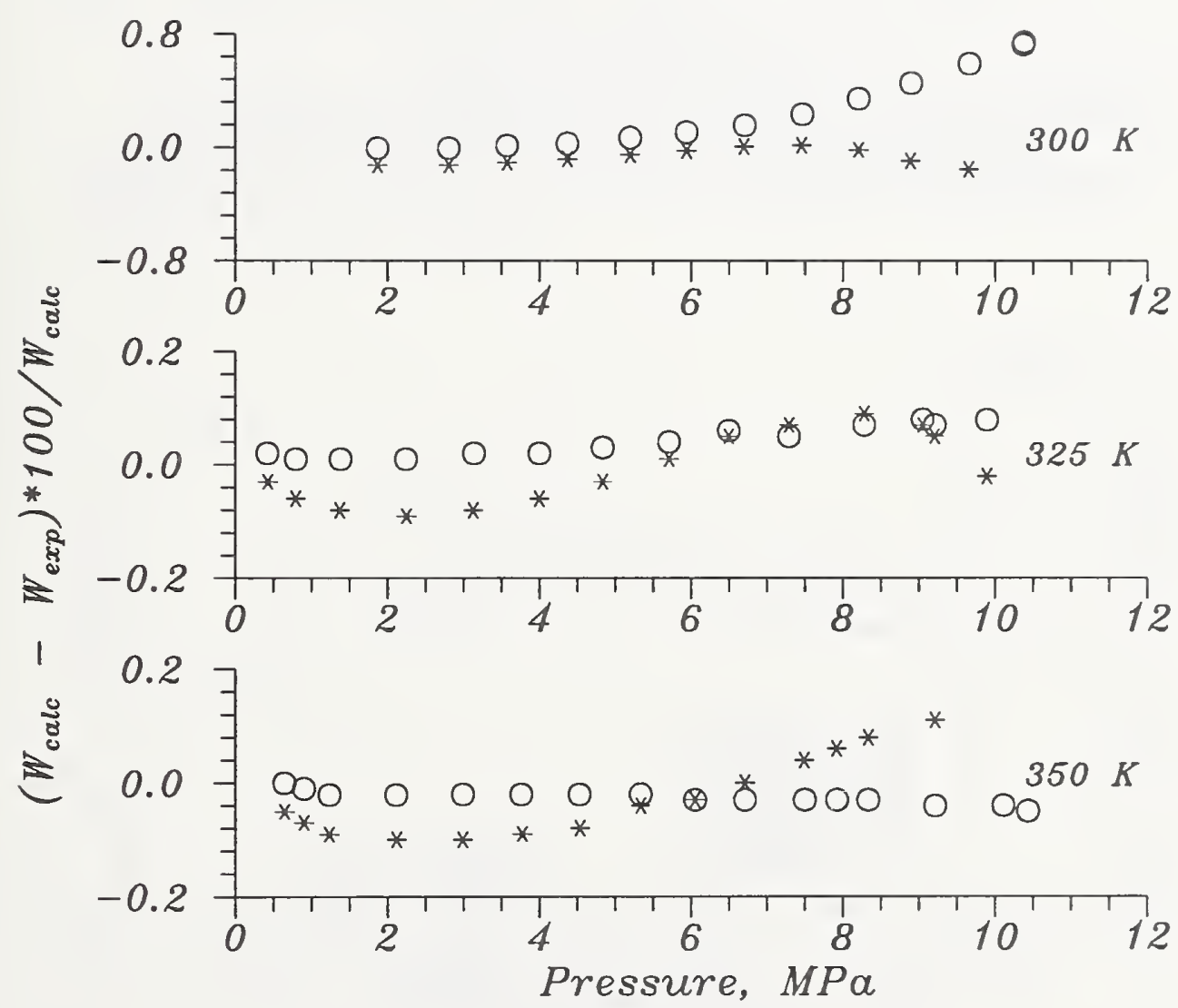

Figure 37. Deviations of speed of sound computed by $A G A 8$ and NGAS from the experimental values for the Statoil Statvordgass mixture. 


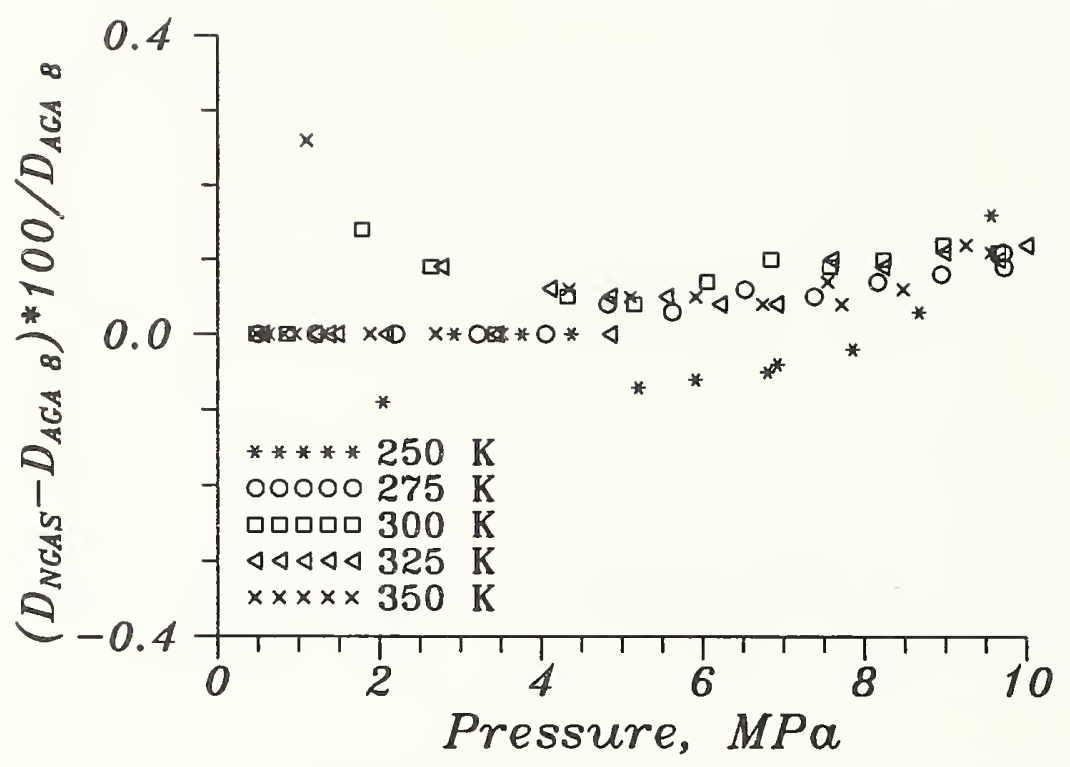

Figure 38. Deviations of the densities computed by NGAS from those computed using $A G A 8$ for the Gulf Coast mixture.

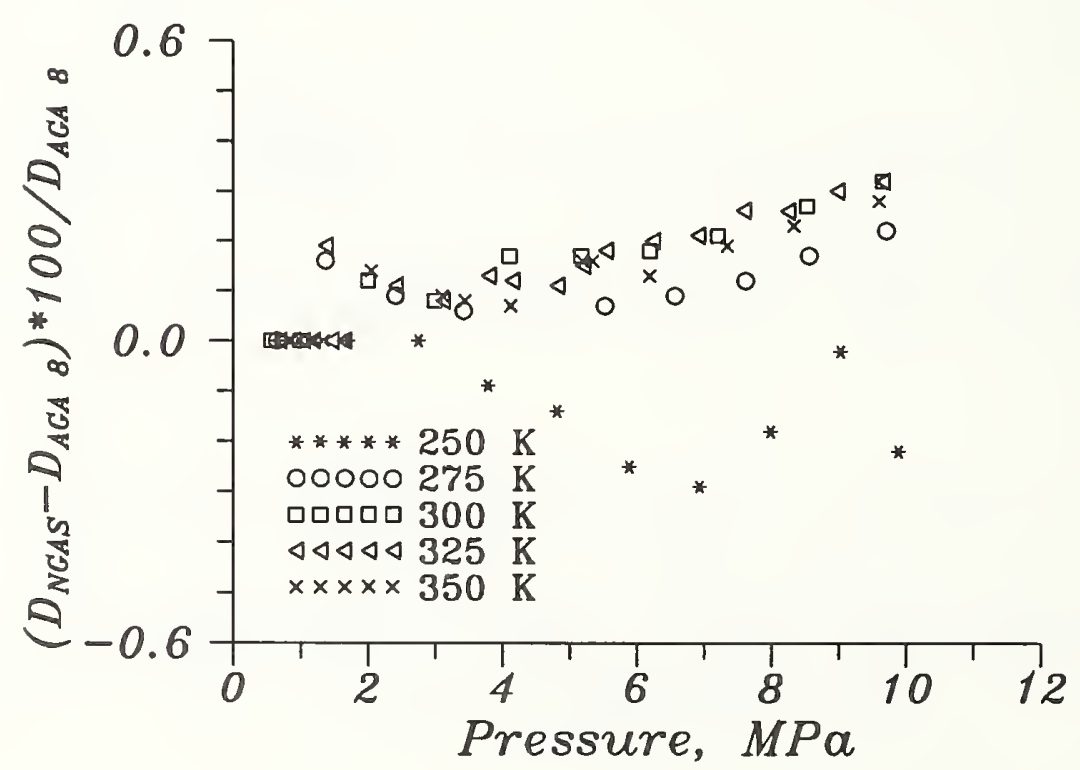

Figure 39. Deviations of the densities computed by NGAS from those computed using $A G A \&$ for the Amarillo mixture. 


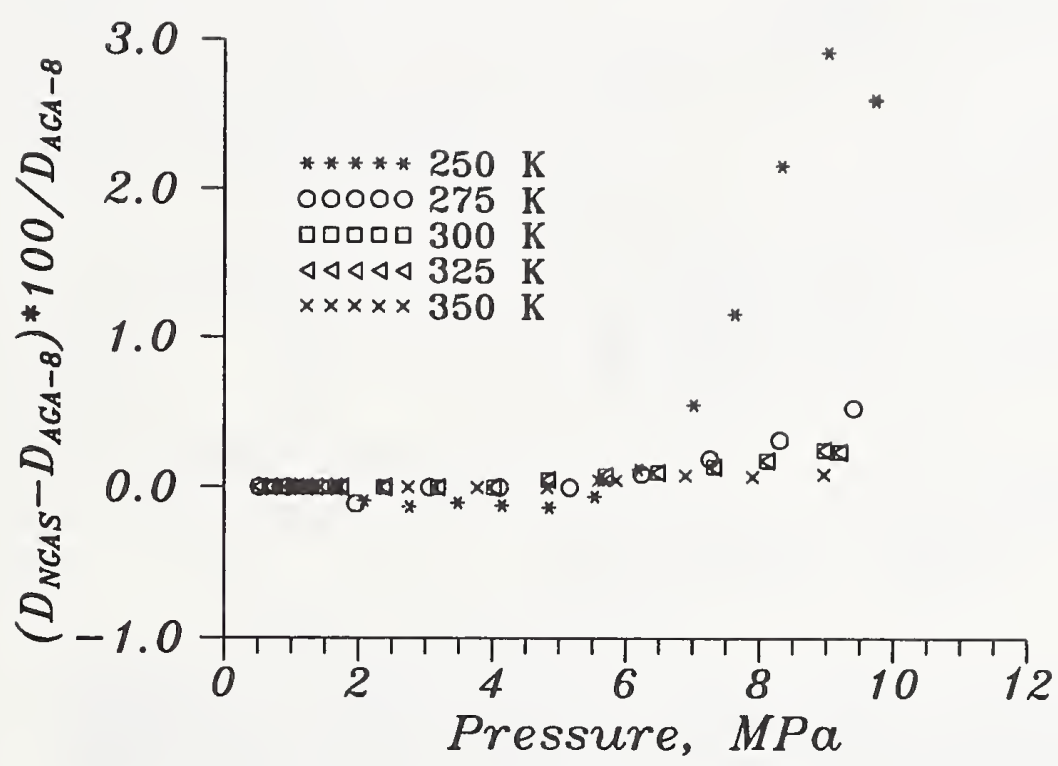

Figure 40. Deviations of the densities computed by NGAS from those computed using AGA 8 for the Statoil dry gas mixture.

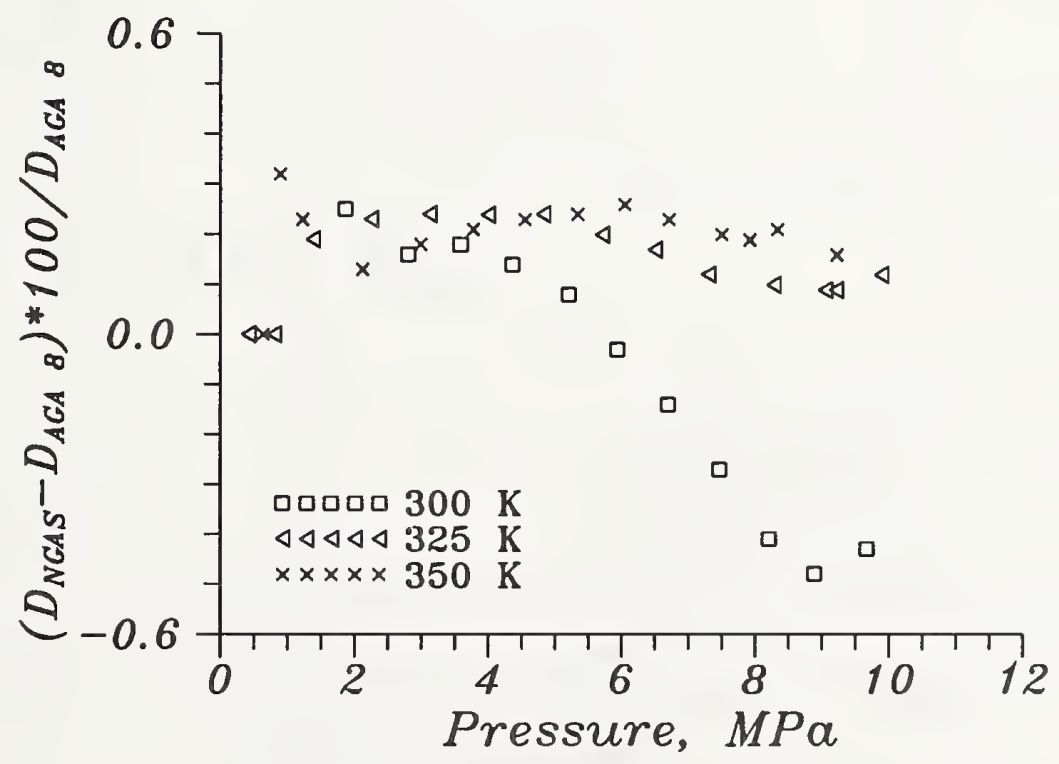

Figure 41. Deviations of the densities computed by NGAS from those computed using $A G A \&$ for the Statoil Statvordgass mixture. 


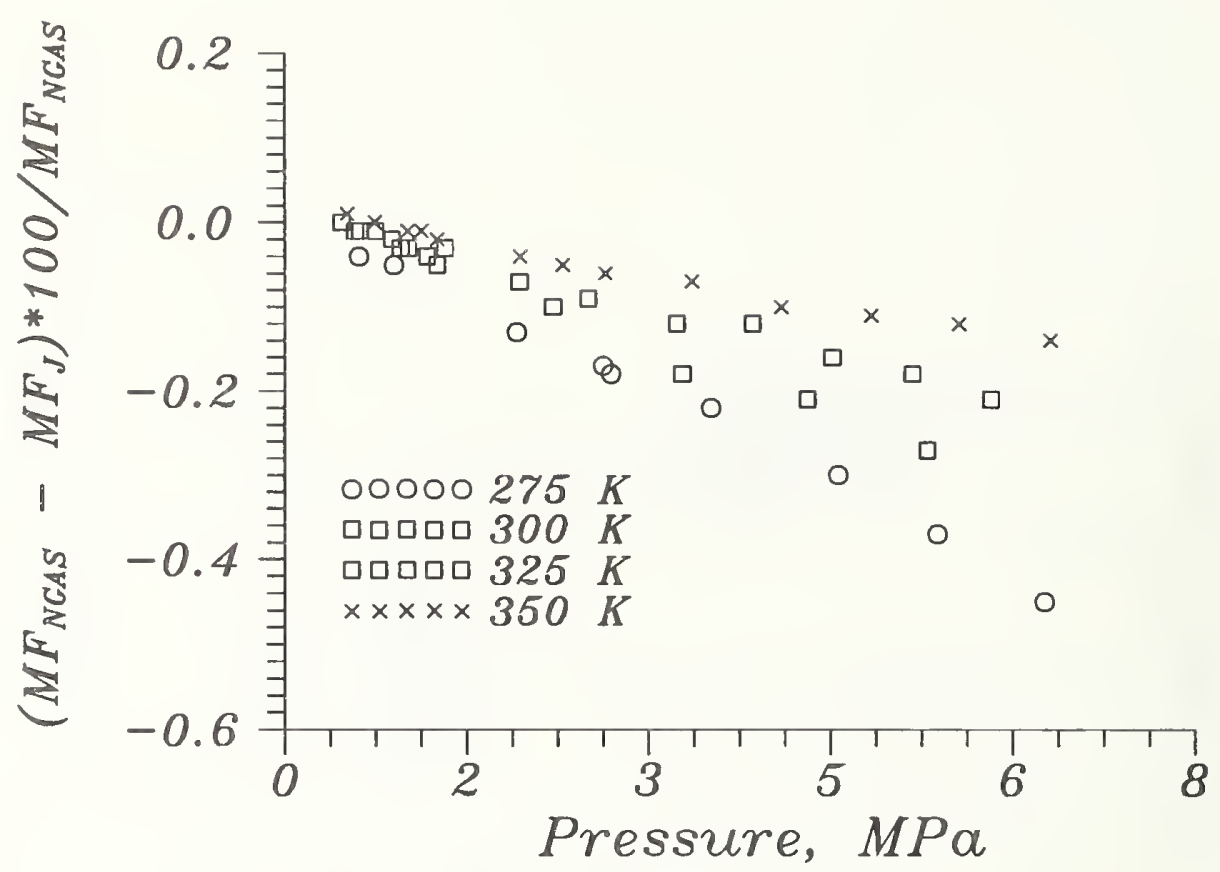

Figure 42. Deviations of mass flow computed by the Johnson equation from that computed by NGAS for methane 0.95 - ethane 0.05 .

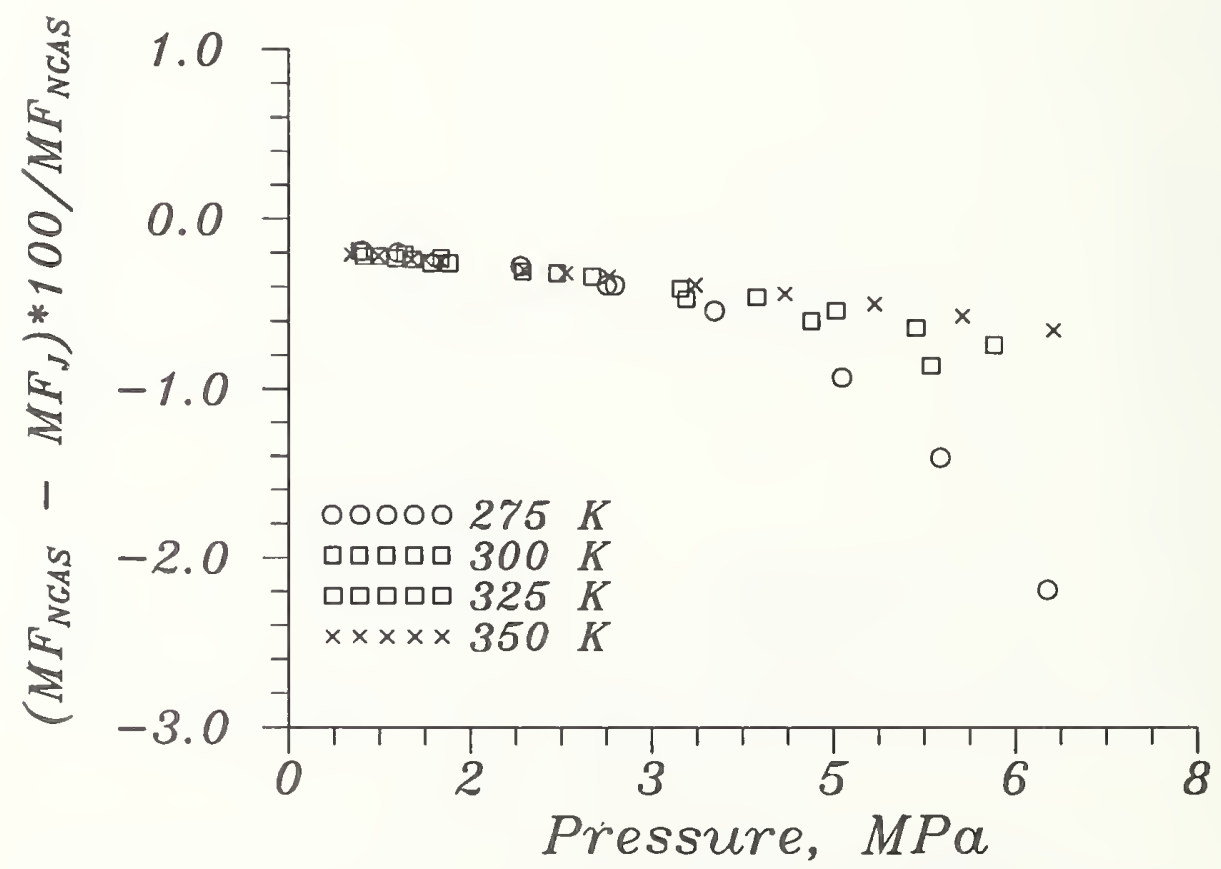

Figure 43. Deviations of mass flow computed by the Johnson equation from that computed by NGAS for methane 0.70 - ethane 0.30 . 


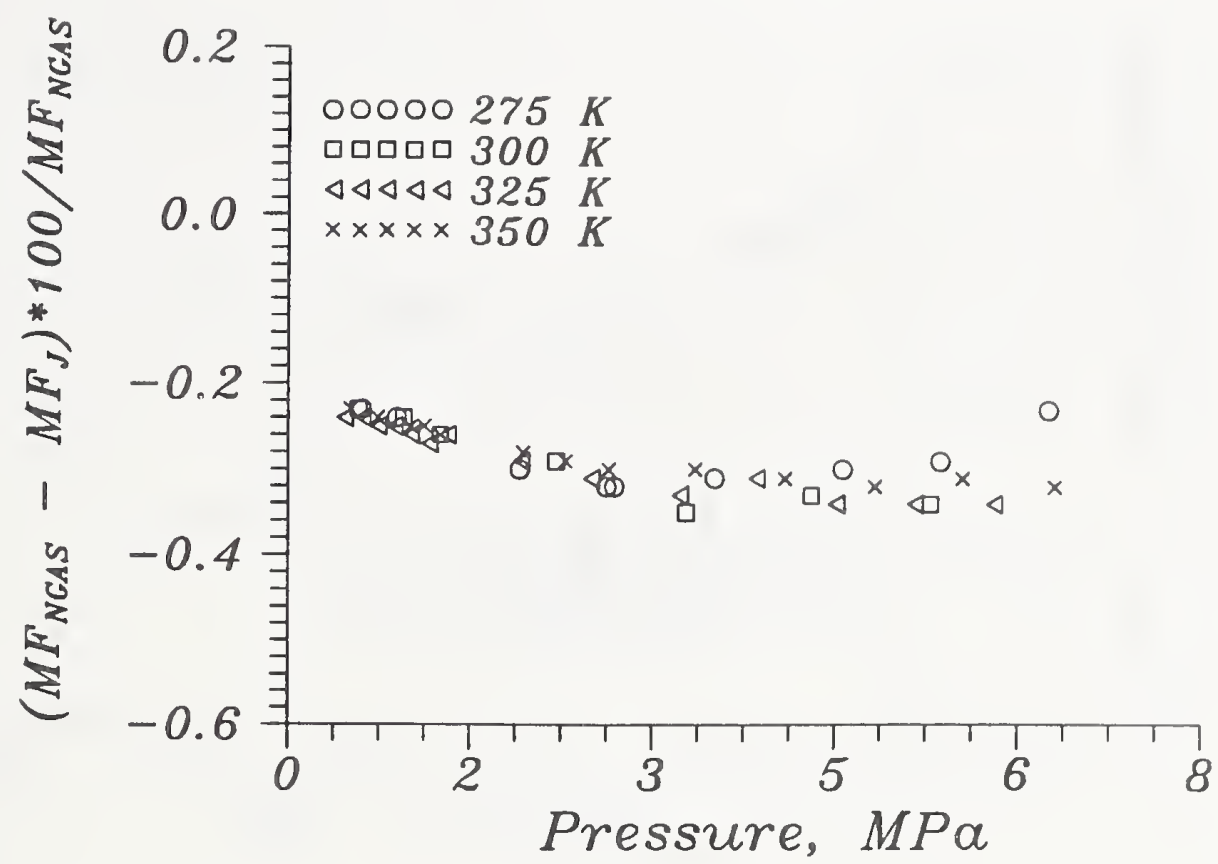

Figure 44. Deviations of mass flow computed by the Johnson equation from that computed by NGAS for methane 0.95 - carbon dioxide 0.05 .

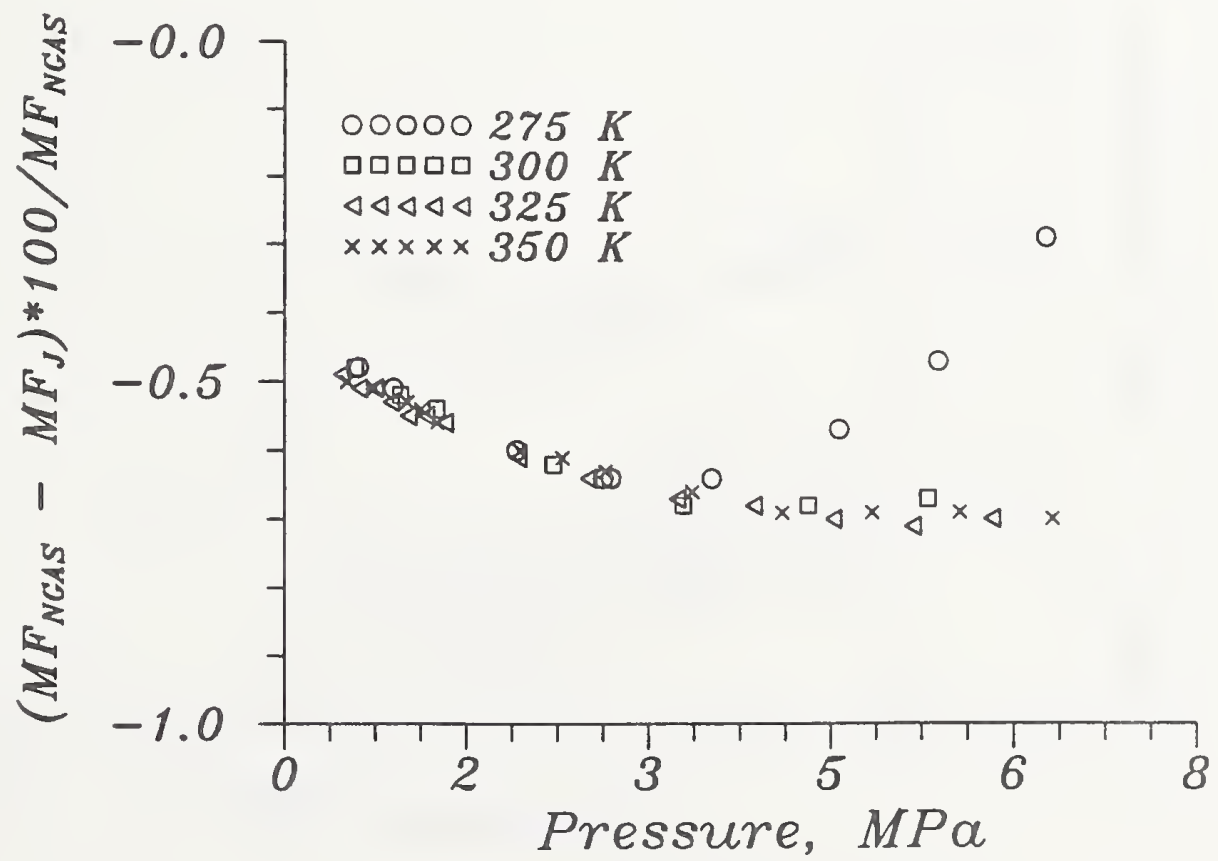

Figure 45. Deviations of mass flow computed by the Johnson equation from that computed by NGAS for methane 0.90 - carbon dioxide 0.10 . 


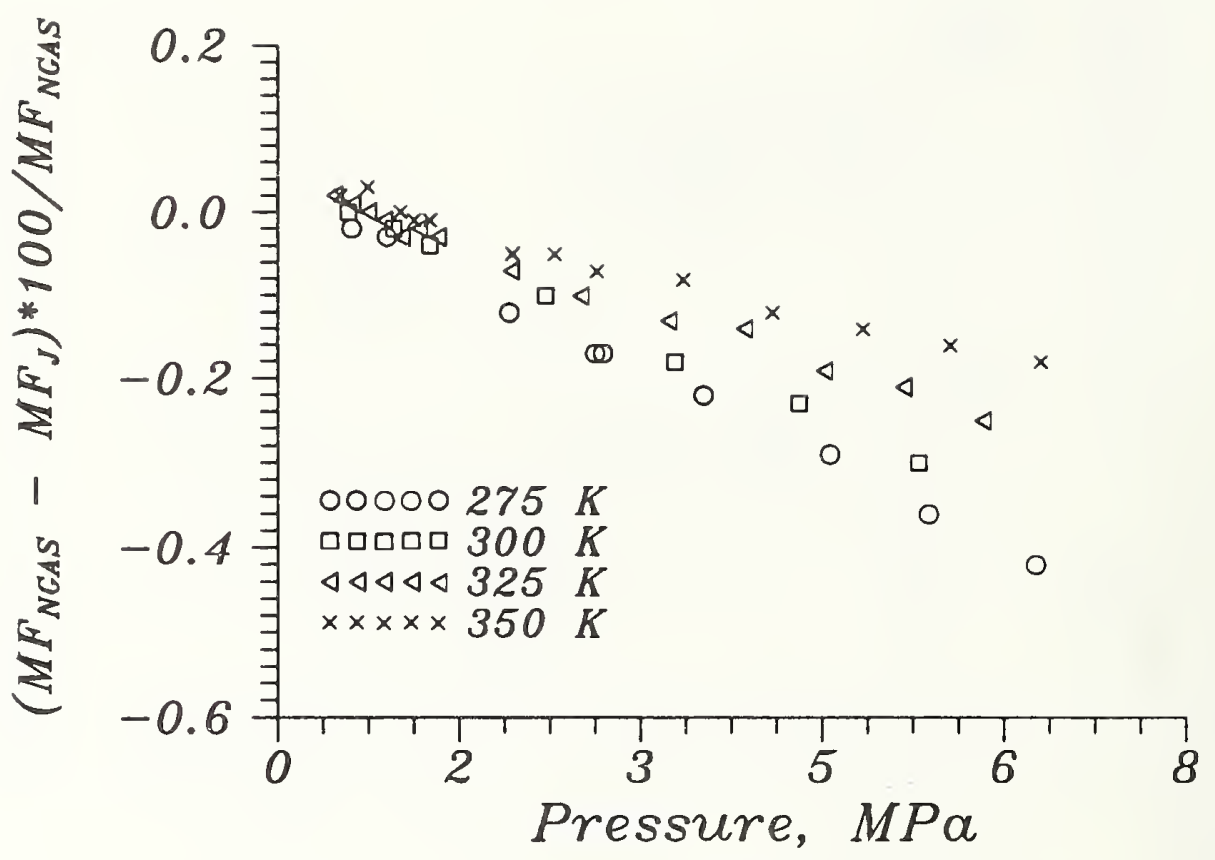

Figure 46. Deviations of mass flow computed by the Johnson equation from that computed by NGAS for methane 0.95 - nitrogen 0.05 .

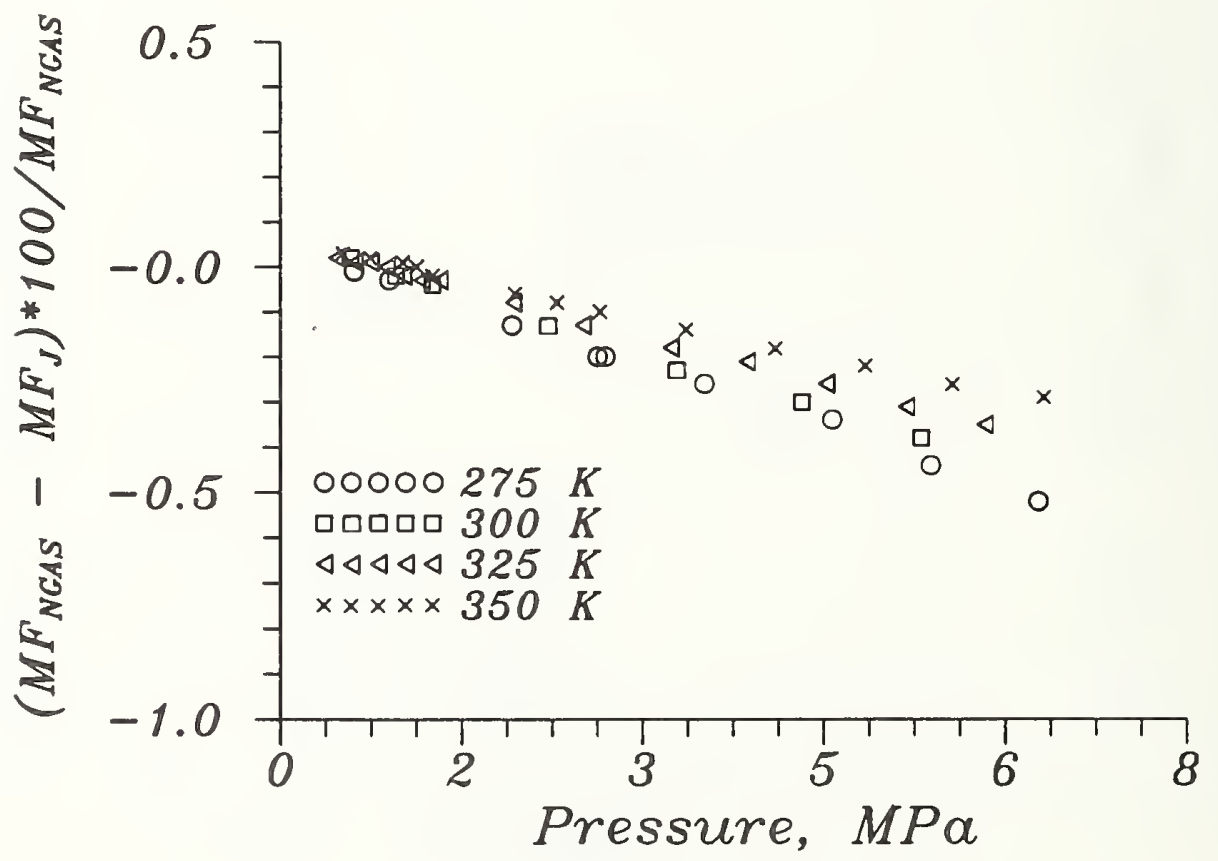

Figure 47. Deviations of mass flow computed by the Johnson equation from that computed by NGAS for methane 0.90 - nitrogen 0.10 . 


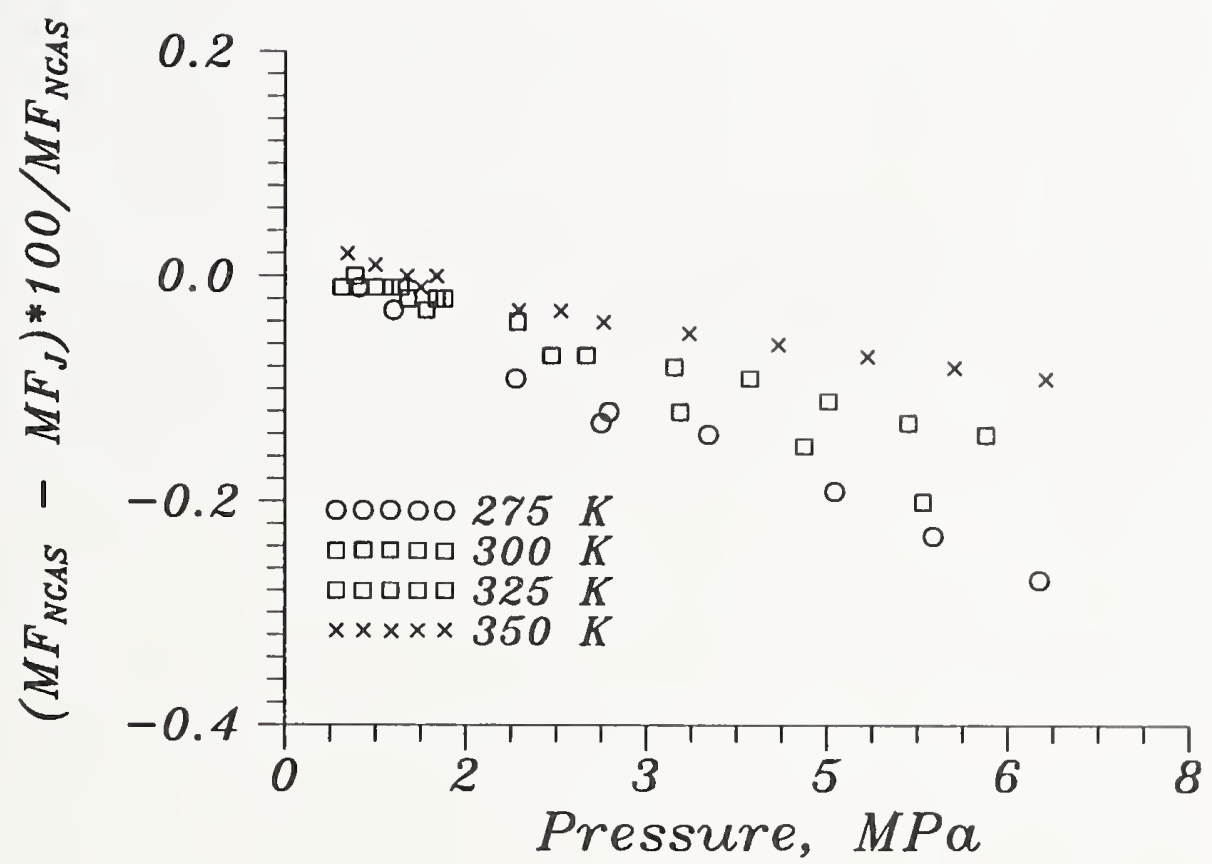

Figure 48. Deviations of mass flow computed by the Johnson equation from that computed by NGAS for the Gulf Coast mixture.

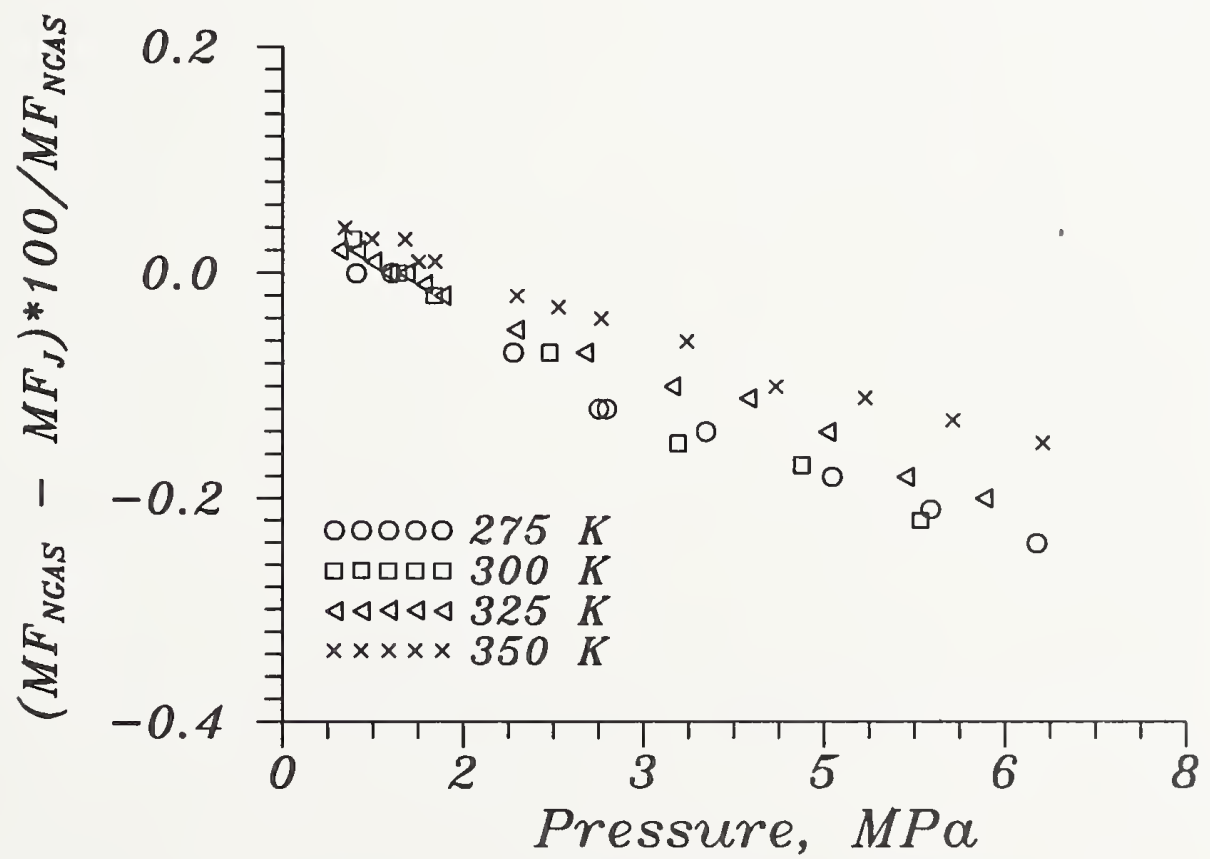

Figure 49. Deviations of mass flow computed by the Johnson equation from that computed by NGAS for the Amarillo mixture. 


Journal of Research of the National Institute of Standards and Technology-Reports NIST research and development in those disciplines of the physical and engineering sciences in which the Institute is active. These include physics, chemistry, engineering, mathematics, and computer sciences. Papers cover a broad range of subjects, with major emphasis on measurement methodology and the basic technology underlying standardization. Also included from time to time are survey articles on topics closely related to the Institute's technical and scientific programs. Issued six times a year.

\section{Nonperiodicals}

Monographs-Major contributions to the technical literature on various subjects related to the Institute's scientific and technical activities.

Handbooks - Recommended codes of engineering and industrial practice (including safety codes) developed in cooperation with interested industries, professional organizations, and regulatory bodies.

Special Publications - Include proceedings of conferences sponsored by NIST, NIST annual reports, and other special publications appropriate to this grouping such as wall charts, pocket cards, and bibliographies.

Applied Mathematics Series-Mathernatical tables, manuals, and studies of special interest to physicists, engineers, chemists, biologists, mathematicians, computer programmers, and others engaged in scientific and technical work.

National Standard Reference Data Series-Provides quantitative data on the physical and chemical properties of materials, compiled from the world's literature and critically evaluated. Developed under a worldwide program coordinated by NIST under the authority of the National Standard Data Act (Public Law 90-396). NOTE: The Journal of Physical and Chemical Reference Data (JPCRD) is published bimonthly for NIST by the American Chemical Society (ACS) and the American Institute of Physics (AIP). Subscriptions, reprints, and supplements are available from ACS, 1155 Sixteenth St., NW, Washington, DC 20056.

Building Science Series-Disseminates technical information developed at the Institute on building materials, components, systems, and whole structures. The series presents research results, test methods, and performance criteria related to the structural and environmental functions and the durability and safety characteristics of building elements and systems.

Technical Notes-Studies or reports which are complete in themselves but restrictive in their treatment of a subject. Analogous to monographs but not so comprehensive in scope or definitive in treatment of the subject area. Often serve as a vehicle for final reports of work performed at NIST under the sponsorship of other government agencies.

Voluntary Product Standards-Developed under procedures published by the Department of Commerce in Part 10, Title 15, of the Code of Federal Regulations. The standards establish nationally recognized requirements for products, and provide all concerned interests with a basis for common understanding of the characteristics of the products. NIST administers this program in support of the efforts of private-sector standardizing organizations.

Consumer Information Series - Practical information, based on NIST research and experience, covering areas of interest to the consumer. Easily understandable language and illustrations provide useful background knowledge for shopping in today's technological marketplace. Order the above NIST publications from: Superintendent of Documents, Government Printing Office, Washington, DC 20402.

Order the following NIST publications-FIPS and NISTIRs-from the National Technical Information Service, Springfield, VA 22161.

Federal Information Processing Standards Publications (FIPS PUB) - Publications in this series collectively constitute the Federal Information Processing Standards Register. The Register serves as the official source of information in the Federal Government regarding standards issued by NIST pursuant to the Federal Property and Administrative Services Act of 1949 as amended, Public Law 89-306 (79 Stat. 1127), and as implemented by Executive Order 11717 (38 FR 12315, dated May 11, 1973) and Part 6 of Title 15 CFR (Code of Federal Regulations).

NIST Interagency Reports (NISTIR) - A special series of interim or final reports on work performed by NIST for outside sponsors (both government and non-government). In general, initial distribution is handled by the sponsor; public distribution is by the National Technical Information Service, Springfield, VA 22161, in paper copy or microfiche form. 


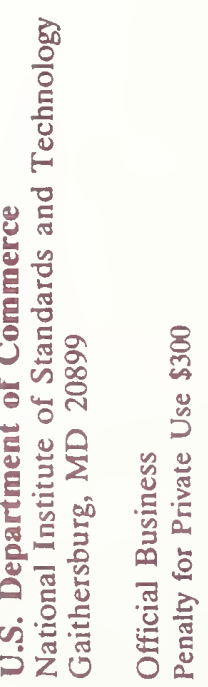

